\title{
Semi-global simulations of the magneto-rotational instability in core collapse supernovae
}

\author{
M. Obergaulinger ${ }^{1}$, P. Cerdá-Durán ${ }^{1}$, E. Müller ${ }^{1}$, and M. A. Aloy ${ }^{2}$ \\ ${ }^{1}$ Max-Planck-Institut für Astrophysik, Garching bei München, Germany \\ e-mail: mobergau@MPA-Garching.MPG. de \\ 2 Departamento de Astronomía y Astrofísica, Universidad de Valencia, 46100 Burjassot, Spain
}

Received 10 November 2008 / Accepted 16 February 2009

\begin{abstract}
Context. Possible effects of magnetic fields in core collapse supernovae rely on an efficient amplification of the weak pre-collapse fields. It has been suggested that the magneto-rotational instability (MRI) leads to a rapid growth for these weak seed fields. Although plenty of MRI studies exist for accretion disks, the application of their results to core collapse supernovae is inhibited as the physics of supernova cores is substantially different from that of accretion discs.

Aims. We address the problem of growth and saturation of the MRI in core collapse supernovae by studying its evolution by means of semi-global simulations, which combine elements of global and local simulations by taking the presence of global background gradients into account and using a local computational grid. We investigate, in particular, the termination of the growth of the MRI and the properties of the turbulence in the saturated state.

Methods. We analyze the dispersion relation of the MRI to identify different regimes of the instability. This analysis is complemented by semi-global ideal MHD simulations, where we consider core matter in a local computational box (size $\sim 1 \mathrm{~km}$ ) rotating at subKeplerian velocity and where we allow for the presence of a radial entropy gradient, but neglect neutrino radiation.

Results. We identify six regimes of the MRI depending on the ratio of the entropy and angular velocity gradient. Our numerical models confirm the instability criteria and growth rates for all regimes relevant to core-collapse supernovae. The MRI grows exponentially on time scales of milliseconds, the flow and magnetic field geometries being dominated by channel flows. We find MHD turbulence and efficient transport of angular momentum. The MRI growth ceases once the channels are disrupted by resistive instabilities (stemming from to the finite conductivity of the numerical code), and MHD turbulence sets in. From an analysis of the growth rates of the resistive instabilities, we deduce scaling laws for the termination amplitude of the MRI, which agree well with our numerical models. We determine the dependence of the development of large-scale coherent flow structures in the saturated state on the aspect ratio of the simulation boxes.

Conclusions. The MRI can grow rapidly under the conditions considered here, i.e., a rapidly rotating core in hydrostatic equilibrium, possibly endowed with a nonvanishing entropy gradient, leading to fields exceeding $10^{15} \mathrm{G}$. More investigations are required to cover the parameter space more comprehensively and to include more physical effects.
\end{abstract}

Key words. magnetohydrodynamics (MHD) - instabilities - stars: supernovae: general - stars: magnetic fields

\section{Introduction}

The magneto-rotational instability (MRI) (Balbus \& Hawley $1991)$ is a local linear instability of weakly magnetized differentially rotating fluids. A large number of analytic, as well as numerical studies support the assertion that the MRI is the main agent for exciting turbulence in Keplerian accretion disks (for a review, see, e.g., Balbus \& Hawley 1998). The MRI amplifies seed perturbations exponentially with time until turbulence sets in. In the turbulent state, the magnetic field, $\boldsymbol{b}$, gives rise to a non-vanishing (spatial and temporal) mean Maxwell stress tensor $M_{i j}=b_{i} b_{j}$. Simulations of accretion disks show a high negative mean value of the component $M_{\varpi \phi}$ (where $\varpi$ and $\phi$ are the radial and azimuthal coordinate of a cylindrical coordinate system), which gives rise to an efficient outward transport of angular momentum.

Akiyama et al. (2003) pointed out that the layers surrounding the nascent proto-neutron star quite generically fulfill the MRI instability criteria. Consequently, any (weak) seed magnetic field will be amplified exponentially. In the saturated state of the MRI instability, sustained magneto-hydrodynamic turbulence might then provide an efficient means for an angular momentum redistribution and for the conversion of rotational energy into thermal energy of the gas. Imparting additional thermal energy into the post-shock stellar matter the MRI might thus be important for the currently favored neutrino-driven core collapse supernova explosion mechanism (e.g. Thompson et al. 2005; Janka et al. 2007), although possibly only for rapidly and strongly differentially rotating progenitors. Furthermore, the growth of the magnetic field resulting from the MRI may provide the adequate physical conditions in the collapsed core to launch bipolar outflows, which result in gamma-ray bursts (Aloy \& Obergaulinger 2007). As the physical conditions in accretion disks and stars differ significantly, and as only a few analytic studies of the MRI in stars exist (e.g., Acheson 1978), it remains unclear whether existing results on the MRI in disks apply to stars, and particularly to supernovae, as well.

Numerical simulations of the MRI face a severe problem, since the growth rate of MRI-unstable modes depends on the product of the initial field strength and the wave number of the mode. For a weak field, only fairly short modes grow rapidly. Simulations of astrophysical flows, on the other hand, often fail 
to resolve just those modes, as it would require prohibitively high computational costs to cover spatial scales ranging from the global extent of the astrophysical system (which may be much larger than the MRI-unstable region) down to the wavelengths of the fastest growing MRI modes. This dependence of the growth rate on the wavelength of the mode suggests a twofold approximate numerical approach: one either performs simulations which properly cover the global scales of the astrophysical system foregoing to resolve the small scales set by the wavelengths of the fastest growing MRI modes (global simulations), or vice versa (local simulations).

Local simulations evolve only a small part of the entire MRI-unstable system, known as the shearing box. However, information on the scales exceeding the size of the computational grid has to be provided using suitably chosen boundary conditions. No unique recipe exists for this procedure, but the use of reflecting and periodic boundaries is a common practice. In most studies of accretion disks, the boundary conditions of Hawley \& Balbus (1992) are used in radial direction, which are essentially periodic boundary conditions but account also for the relative shear between the inner and the outer radial edge of the grid. They are often combined with a Galilei transformation into a frame of reference co-rotating at the mean angular velocity of the shearing box, and a linearizion of the angular velocity within the box. Local (shearing box) simulations using this kind of boundary treatment are commonly called shearing-sheet simulations.

The general drawback of local simulations obviously lies in their inability to account accurately for large-scale phenomena. In addition, there is only a limited possibility to model global gradients other than differential rotation in shearing-sheet simulations. Independent of the boundary treatment only modes with a wavelength less than the size of the grid can be excited, i.e. modes with a wavelength comparable to the dimensions of the whole system cannot develop. Consequently, MRI-driven turbulence may saturate at a level determined (at least partially) by numerical rather than (only) by physical parameters. A careful analysis is necessary to disentangle the respective influence (see e.g., Pessah et al. 2007; Fromang \& Papaloizou 2007; Regev \& Umurhan 2008).

Global simulations, on the other hand, follow the evolution of the entire system, albeit with a much coarser resolution than local ones. Thus, they can account for the large-scale structure of stars and disks, for the back-reaction of the MRI instigated turbulence on the global flow, and allow one to draw conclusions on how the saturated state depends on global properties of the system, e.g., the density or pressure stratification. However, foregoing the ability to resolve short-wavelength modes, the growth of the MRI will be underestimated or suppressed even entirely. In many applications of numerical analysis, it is possible to use suitable models for the unresolved physics on sub-grid scales, e.g., sub-grid diffusivity. This requires a good knowledge of the physics on these scales, and is facilitated greatly if processes at the unresolved scales act merely as a sink for kinetic or magnetic energy cascading down from the integral scale. During the growth of the MRI, however, the power shifts gradually from short to long modes. Thus, sub-grid models for global MRI simulations tend to be complex, and are not used widely.

As a remedy for this problem, global simulations may be performed using unrealistically strong initial fields to guarantee that the fastest growing MRI modes are resolved numerically. This approach presumes that the unresolved MRI modes are able to amplify the much weaker actual initial fields to the field strengths used as initial value in global simulations. This assumption can be justified, if the MRI acting on the unresolved scales saturates at the initial field strengths imposed in global simulations, i.e., if rapid amplification by the MRI takes place over many orders of magnitude. However, this can only be proven by high-resolution local simulations. Enhancing the initial magnetic field by a constant factor throughout the computational domain, as it is often done in global simulations, is problematic as the MRI is a local instability, i.e. it is not expected to cause a constant amplification of the field everywhere. The ambiguities regarding differences between the topology of this artificially enhanced field and that of a field amplified locally by the MRI add to the uncertainties clouding the influence of magnetic fields on the overall dynamics.

Both the local and the global numerical approach has been used for studying the MRI in accretion disks, and this combined effort has led to the rapid development of the field. Simulations of the MRI in core collapse supernovae, on the other hand, have not yet reached this advanced stage, mainly because of the weakness of the initial field of the progenitors. According to current stellar evolution models (Heger et al. 2005), the canonical precollapse magnetic fields are so weak that they are unable to affect the dynamics of the explosion unless they are amplified strongly. Correspondingly, the wavelengths of the fastest growing MRI modes are approximately a few meters at most ${ }^{1}$. Thus, the possible importance of MHD effects in core collapse supernovae depends on the existence of mechanisms which can amplify the field efficiently during core collapse and the post-bounce phase. The timescale available for the growth of the magnetic field is set by the time required to turn the accretion of matter onto the proto-neutron star into an explosion, i.e., a few hundreds of milliseconds. As already mentioned above Akiyama et al. (2003) suggested that the MRI might provide this mechanism. They estimated the saturation field strength to be $10^{15}-10^{16} \mathrm{G}$, i.e., clearly in excess of the artificially enhanced initial field strengths used in global simulations.

Up to now, there exist only global simulations of MHD core collapse supernovae, which evolve the entire core of a massive star through gravitational collapse, bounce, and explosion (e.g., Kotake et al. 2004; Yamada \& Sawai 2004; Takiwaki et al. 2004; Obergaulinger et al. 2006b,a; Burrows et al. 2007). These global simulations fail to find the MRI unless they employ drastically stronger initial fields. Obergaulinger et al. (2006b,a), e.g., require a pre-collapse field strength exceeding $10^{12} \mathrm{G}$ to resolve the MRI in the post-bounce state. The rationale behind the artificially increased initial field strengths is that, once triggered by the differential rotation in the proto-neutron star, the MRI will exponentially amplify a much weaker seed field up to the values used in the simulations.

Due to the lack of local simulations, the importance of the MRI in MHD core collapse models remains unclear. As a first step to resolve this issue, we have performed high-resolution simulations of small parts of simplified post-bounce, rotating, magnetized cores. We have used a recently developed highresolution MHD code, and employed shearing-disk boundary conditions (Klahr \& Bodenheimer 2003). These boundary conditions derive from the shearing-sheet boundary conditions of Hawley \& Balbus (1992), but allow one to consider global gradients of, e.g., density or entropy. Combining elements of global and local simulations, viz. the presence of global background gradients, and a high-resolution local grid, we find it justified to call our approach semi-global (for more details see Sect. 3.2).

\footnotetext{
1 Note, however, that these predictions still involve uncertainties, and hence rare, but much more strongly magnetized progenitors cannot be excluded presently.
} 
Differences in the physical conditions in disks and stars impede the direct application of the MRI results from accretion disks to supernovae. Most obviously, the geometry of both systems differs strongly. Furthermore, while accretion discs are stabilized against gravity by (Keplerian) rotation, stars are supported mainly by pressure gradients, with only a minor contribution from rotation, i.e. thermal stratification is much more important in stars than in disks. Thus, entropy gradients can stabilize an MRI-unstable region or modify the instability in convectively unstable regions. Consequently, the problem of the MRI in core collapse supernovae has to be addressed by simulations accounting for their specific properties, which is the goal of this study. We investigate the growth of the MRI from initial fields comparable to the ones expected from realistic stellar evolution modeling, and we seek to probe the possibility of MRI-driven field amplification under typical conditions of supernova cores and on timescales similar to the dynamic times of the system, (i.e., a few tens of milliseconds). Apart from the restrictions inherent to local and semi-global simulations, several simplifications limit our approach: we use simplified initial equilibrium models, a simplified equation of state, and neglect neutrino heating and cooling. The main physical questions that we try to address are: (i) does the MRI grow on sufficiently short time scales to influence the explosion, i.e., within at most $100 \mathrm{~ms}$, given typical post-bounce rotation profiles and magnetic fields?; (ii) How does an entropy gradient affect the growth of the instability?; (iii) How does the saturated state of MRI-driven turbulence depend on these factors? In particular, is the saturation field strength estimated by Akiyama et al. (2003), i.e., the conversion of most of the rotational energy into magnetic energy, realistic?

Analogous questions are studied by local simulations of the MRI in accretion disks. The answers may lead the way to formulate a turbulence model to be used in global simulations. The simplest model would provide a parametrization of the angular momentum transport by an $\alpha$ viscosity (Shakura \& Syunyaev 1973), i.e., a turbulent viscosity proportional to the local sound speed and the pressure scale height. However, despite a large number of local simulations, no unique formulation of an $\alpha$ model for accretion disks has been found up to now. Lacking similar comprehensive local simulations, a turbulence model for the MRI in supernovae is even less conceivable. Our simulations intent to provide only a first step towards these highly desired turbulence models.

The paper is organized as follows: after a discussion of the main properties of the MRI in disks and stars (Sect.2), we outline our numerical method in Sect.3, discuss our results in Sect. 4, and summarize our main results and give conclusions in Sect. 5.

\section{MRI in discs and stars}

\subsection{Physical model}

We work in the limit of ideal magnetohydrodynamics (MHD), solving the the equations of ideal MHD in the presence of an external gravitational potential $\varphi$,

$\partial_{t} \rho+\nabla_{j}\left[\rho v^{j}\right]=0$

$\partial_{t} p^{i}+\nabla_{j}\left[p^{i} v^{j}+P_{\star} \delta^{i j}-b^{i} b^{j}\right]=\rho \nabla_{i} \varphi$

$\partial_{t} e_{\star}+\nabla_{j}\left[\left(e_{\star}+P_{\star}\right) v^{j}-b^{i} v_{i} b^{j}\right]=\rho v^{j} \nabla_{j} \varphi$,

$\partial_{t} \boldsymbol{b}=-c \boldsymbol{\nabla} \times \boldsymbol{E}$, $\nabla_{j} b^{j}=0$

Here, $\rho, \boldsymbol{p}, \boldsymbol{v}$, and $e_{\star}$ denote the mass density, momentum density, velocity, total-energy density, of the gas, respectively; $\boldsymbol{b}$ is the magnetic field. The total-energy density and the total pressure, $P_{\star}$, are composed of fluid and magnetic contributions: $e_{\star}=\varepsilon+$ $\frac{1}{2} \rho \boldsymbol{v}^{2}+\frac{1}{2} \boldsymbol{b}^{2}$ and $P_{\star}=P+\frac{1}{2} \boldsymbol{b}^{2}$ with the internal-energy density $\varepsilon$ and the gas pressure $P=P(\rho, \varepsilon, \ldots)$. The electric field, $\boldsymbol{E}$, is given by $\boldsymbol{E}=-\frac{v}{c} \times \boldsymbol{b}$. Here, $c=2.998 \times 10^{10} \mathrm{~cm} \mathrm{~s}^{-1}$ is the speed of light in vacuum and Einstein's summation convention applies.

We use the hybrid equation of state (EOS) due to Keil et al. (1996) as a rough model for neutron-star matter. Following this EOS, the total gas pressure, $P$, consists of a barotropic part, $P_{\mathrm{b}}$, and a thermal part, $P_{\text {th. }}$. The two parts are given by

$P_{\mathrm{b}}=\kappa \rho^{\Gamma_{\mathrm{b}}}$,

$P_{\text {th }}=\left(\Gamma_{\text {th }}-1\right) \varepsilon_{\text {th }}$.

Here, $\Gamma_{\mathrm{b}}$ and $\kappa$ refer to the barotropic adiabatic index, and the polytropic constant of the EOS, respectively; $\varepsilon_{\mathrm{th}}=\varepsilon-P_{\mathrm{b}} /\left(\Gamma_{\mathrm{b}}-1\right)$ is the thermal part of the internal energy, and $\Gamma_{\text {th }}$ the corresponding adiabatic index. Please note that we consider only subnuclear densities, $\rho<\rho_{\text {nuc }}=2 \times 10^{14} \mathrm{~g} \mathrm{~cm}^{-3}$ here since the maximum density reached in our models is a few times $10^{13} \mathrm{~g} \mathrm{~cm}^{-3}$.

We define a pseudo-entropy $S$ for this equation of state by

$S=\frac{P_{\mathrm{th}}}{P_{\mathrm{b}}}$

In the Schwarzschild criterion for convective stability (see below), this quantity appears instead of the entropy of, e.g., an ideal gas.

A few quantities used frequently in the remainder of this paper are:

1. the Alfvén velocity

$c_{\mathrm{A}}=\frac{|\boldsymbol{b}|}{\sqrt{\rho}}$

2. the (local) magnetic energy density

$\mathrm{e}_{\mathrm{mag}}=\frac{\boldsymbol{b}^{2}}{2}$

and the corresponding volumetric mean value

$e_{\mathrm{mag}}=\frac{1}{\mathcal{V}} \int \mathrm{d} \mathcal{V} \mathrm{e}_{\mathrm{mag}}$,

3. the (local) Maxwell stress tensor

$M_{i j}=b_{i} b_{j}$,

and the corresponding volumetric mean value

$M_{i j}=\frac{1}{\mathcal{V}} \int \mathrm{d} \mathcal{V} M_{i j}$

We will use most frequently the component $M_{\varpi \phi}$ which governs the transport of angular momentum in radial direction, and we will sometimes refer to this component as the Maxwell stress for short. 


\subsection{General properties of the MRI}

The stability criteria for the MRI was first discovered by Velikhov (1959); Chandrasekhar (1960) and further discussed by Balbus \& Hawley (1991) in a series of papers. These authors analyze wave-like (WKB) perturbations of the form exp $[i(\boldsymbol{k} \cdot \boldsymbol{r}+\omega t)]$ in a background equilibrium of the MHD equations. For convenience cylindrical coordinates $(\varpi, \phi, z)$ are used in the following equations. From the dispersion relation, they derive the criteria for exponential growth and, if applicable, the growth rates of WKB modes. Because the main astrophysical context of this series of papers is accretion discs, some assumptions are made which considerably simplify the analysis: i) weak magnetic fields where $\left|\boldsymbol{v}_{\mathrm{A}}\right| \ll \min \left(c_{\mathrm{s}},\left|v_{\varphi}\right|\right)$; ii) incompressible gas (Boussinesq approximation), and iii) angular velocity constant on cylinders, $\Omega(\varpi)$. The discussion is mostly restricted to thin discs (i.e., to equatorial regions and to a Keplerian rotation law) and 5/3-polytropes. Under these assumptions the stability criterion for a differentially rotating magnetized fluid is (Balbus \& Hawley 1991)

$\mathcal{R}_{\varpi} \equiv \varpi \partial_{\varpi} \Omega^{2}>0$.

If the criterion is not fulfilled only modes with (dimensionless) wavenumber

$\hat{k}<\sqrt{-\hat{\mathcal{R}}_{\varpi}}=\hat{k}_{\text {crit }}$

are unstable, where $\hat{k} \equiv \boldsymbol{k} \cdot \boldsymbol{v}_{\mathrm{A}} / \Omega, \hat{k}_{\text {crit }} \equiv \boldsymbol{k}_{\text {crit }} \cdot \boldsymbol{v}_{\mathrm{A}} / \Omega$ and $\hat{\mathcal{R}}_{\varpi} \equiv$ $\mathcal{R}_{\varpi} / \Omega^{2}$. The (dimensional) growth rate of the fastest growing mode is (Balbus \& Hawley 1992)

$\hat{\omega}_{\mathrm{FGM}} \equiv \omega_{\mathrm{FGM}} / \Omega=-\hat{\mathcal{R}}_{\varpi} / 4$

which is independent of the magnetic field and corresponds to (dimensionless) wave numbers close to $\hat{k}_{\text {crit }}$.

However in the context of core collapse supernovae some of these assumptions do not apply: entropy and composition gradients are important, more general rotation laws $\Omega(\varpi, z)$ have to be considered, and the analysis can no longer be restricted to equatorial regions. In this general case the dispersion relation of WKB modes is (cf. Balbus 1995; Urpin 1996),

$$
\begin{aligned}
\left(\hat{\omega}^{2}-\hat{k}^{2}\right)^{2} & -\left(\hat{\omega}^{2}-\hat{k}^{2}\right)\left(\hat{\omega}_{\mathrm{G}}^{2}+\hat{\omega}_{\mathrm{R}}^{2}+4 \cos ^{2} \theta_{k}\right) \\
& -4 \hat{k}^{2} \cos ^{2} \theta_{k}=0
\end{aligned}
$$

where $\hat{\omega}=\omega / \Omega$ is the dimensionless growth rate of the instability, and $\theta_{k}$ is the angle between $\boldsymbol{k}$ and the $z$-axis. The (dimensionless) frequencies related to buoyancy terms and differential rotation are

$\hat{\omega}_{\mathrm{G}}^{2}=\frac{1}{\Omega^{2}}\left[\mathcal{G} \cdot \mathcal{B}-\frac{(\boldsymbol{k} \cdot \mathcal{B})(\boldsymbol{k} \cdot \mathcal{G})}{k^{2}}\right]$

and

$\hat{\omega}_{\mathrm{R}}^{2}=\frac{1}{\Omega^{2}}\left[\mathcal{R} \cdot \boldsymbol{e}_{\varpi}-\frac{\left(\boldsymbol{k} \cdot \boldsymbol{e}_{\varpi}\right)(\boldsymbol{k} \cdot \mathcal{R})}{k^{2}}\right]$,

respectively, where $\boldsymbol{e}_{\varpi}$ is the unit vector in $\varpi$ - direction,

$$
\begin{aligned}
\mathcal{G} & \equiv \frac{\boldsymbol{\nabla} P}{\rho} \\
\mathcal{R} & \equiv \varpi \nabla \Omega^{2} \\
\mathcal{B} & \equiv \frac{\boldsymbol{\nabla} \rho}{\rho}-\frac{\boldsymbol{\nabla} P}{\Gamma_{1} P}=-\left.\frac{1}{\Gamma_{1}} \frac{\partial \ln P}{\partial s}\right|_{\rho} \boldsymbol{\nabla} s
\end{aligned}
$$

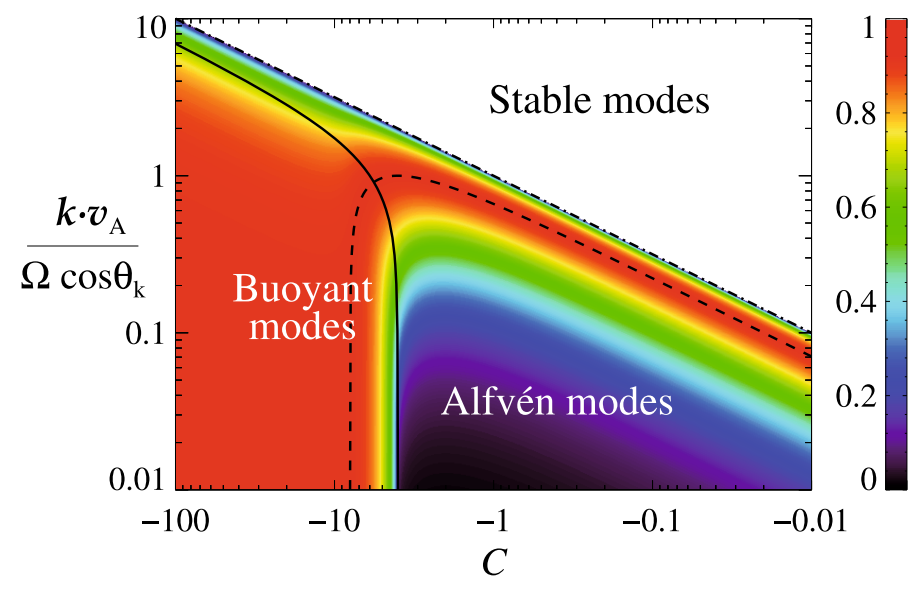

Fig. 1. Imaginary part of the growth rate normalized to the imaginary part of the maximum growth rate, $\mathfrak{J}(\hat{\omega}) / \mathfrak{J}\left(\omega_{\mathrm{FGM}}\right)$ as a function of $C$ and $\hat{k} / \cos \theta_{k} \equiv \boldsymbol{k} \cdot \boldsymbol{v}_{\mathrm{A}} /\left(\Omega \cos \theta_{k}\right)$. The dashed line shows the value of $\hat{k}$ corresponding to the fastest growing mode, $\mathfrak{J}(\hat{\omega}) / \mathfrak{J}\left(\omega_{\hat{\mathrm{FGM}}}\right)=1$, the solid line gives the boundary between the two branches of unstable modes (Alfvén and Buoyant modes), and the dash-dotted line corresponds to the stability limit $\left(\hat{k}=\hat{k}_{\text {crit }}\right)$. For $-4<C<0$ only Alfvén modes appear, with a narrow spectrum of fast growing modes close to $\hat{k}_{\text {crit }}$ (dashdotted line). For $C<-4$ buoyant modes appear and become dominant for $C<-8$. In the latter case the spectrum of fast growing modes is much wider covering the entire region from $\hat{k}_{\text {crit }}$ to 0 .

are the gravitational, rotational, and buoyancy terms, respectively, and $\Gamma_{1} \equiv \partial \ln P /\left.\partial \ln \rho\right|_{s}$. It is convenient for the mode analysis and if $\cos \theta_{k} \neq 0$ to define the quantity

$$
\begin{aligned}
C & \equiv \frac{\hat{\omega}_{\mathrm{G}}^{2}+\hat{\omega}_{\mathrm{R}}^{2}}{\cos ^{2} \theta_{k}} \\
& =\left(\mathcal{G}_{z} \mathcal{B}_{z} \tan ^{2} \theta_{k}-2 \mathcal{B}_{\varpi} \mathcal{G}_{z} \tan \theta_{k}+\mathcal{G}_{\varpi} \mathcal{B}_{\varpi}+\mathcal{R}_{\varpi}\right) / \Omega^{2},
\end{aligned}
$$

where the curl of Eq. (2), i.e. the vorticity equation, has been used to simplify the expression of $C$. Note that this quantity depends on the direction of the perturbation $\theta_{k}$, but not on $k^{2}$ itself. If $\cos \theta_{k}=0$, which corresponds to velocity perturbations parallel to the rotation axis, the value of $C$ diverges, but all length scales and growth rates of the discussion below are finite, and can be computed by taking the limit $\cos \theta_{k} \rightarrow 0$.

In the absence of a magnetic field, i.e. $\hat{k}^{2}=0$, the stability condition is simply $C+4>0$, which is equivalent to the Solberg-Høiland stability criteria for a non-magnetized rotating fluid (Tassoul 1978).

Because we want to study instabilities of magnetized fluids, we consider hereafter only the case $\hat{k} \neq 0$. Then the stability condition is $C>0$, which corresponds to that of Balbus (1995),

$$
\begin{aligned}
& \mathcal{G} \cdot \mathcal{B}+\mathcal{R} \cdot \boldsymbol{e}_{\varpi} \geq 0 \\
& \left(\mathcal{G} \times \boldsymbol{e}_{\varpi}\right) \cdot(\mathcal{B} \times \mathcal{R}) \geq 0 .
\end{aligned}
$$

Modes with wave numbers smaller than the (dimensionless) critical wavenumber

$\hat{k}_{\text {crit }}=\cos \theta_{k} \sqrt{-C}$

are unstable and grow. The critical wavenumber depends on the angle $\theta_{k}$ in a complicated way involving, in general, the rotation profile, $\mathcal{R}$, the thermal structure, $\mathcal{B}$, and the stratification, $\mathcal{G}$.

Two branches of unstable modes arise from the dispersion relation with $\hat{k} \neq 0$ (Urpin 1996): the branch of Alfvén modes appearing for $C<0$, and the branch of buoyant modes which only appear for $C+4<0$ (Fig. 1). 
For a given $\theta_{k}$ the fastest growing mode is obtained from the condition $\partial_{\hat{k}} \hat{\omega}=0$. For $-8<C<0$ it has a (dimensionless) wavenumber

$\hat{k}_{\mathrm{FGM}}=\cos \theta_{k} \frac{\sqrt{-C(C+8)}}{4}$,

and a (dimensionless) growth rate

$\hat{\omega}_{\mathrm{FGM}}=\cos \theta_{k} \frac{\sqrt{-C^{2}}}{4}$.

If $C \leq-8$, the fastest growing mode corresponds to $\hat{k}_{\mathrm{FGM}}=0$, i.e. it is dominated by buoyant modes with a (dimensionless) growth rate

$\hat{\omega}_{\mathrm{FGM}}=\cos \theta_{k} \sqrt{C+4}$.

Thus, there exist two different instability regimes depending on the value of $C$. For $-4<C<0$ only Alfvén modes are possible. This magneto-shear regime was discussed by Balbus \& Hawley (1991). A mixed regime is found for $-8<C<-4$, where both Alfvén and buoyant modes compete. For $C<-8$ the buoyant modes completely dominate the growth of the instability, and this regime is thus called magneto-convective regime. It is similar to the convective regime, but the critical wavenumber is determined by the strength of the magnetic field.

Note that for a given fluid element the behavior of the unstable modes depends on the angle $\theta_{k}$. Thus, different regimes can hold in different directions. To find the absolute fastest growing mode of a fluid element, i.e. not considering a fixed angle $\theta_{k}$, one has to determine the zeros of $\partial \hat{\omega} / \partial \theta_{k}$, which involves the solution of a quartic equation. This fact makes a more detailed study of the instability difficult.

To simplify the analysis, we restrict ourselves in the following discussion to regions near the equator, where it is reasonable to assume only a radial dependence of the hydrodynamic quantities and a vertical magnetic field. Therefore,

$C_{90}=\left(N^{2}+\mathcal{R}_{\varpi}\right) / \Omega^{2}$,

where $N^{2} \equiv \mathcal{B} \cdot \mathcal{G}$ is the square of the Brunt-Väisälä or buoyancy frequency. Because $C_{90}$ does not depend on $\theta_{k}$, all modes of the considered fluid element belong to the same branch of modes, i.e. they are either buoyant modes or Alfvén modes. All modes with wavelengths shorter than

$\lambda_{\text {crit }} \equiv \frac{2 \pi}{k_{\text {crit }}}=\frac{2 \pi\left|\boldsymbol{v}_{\mathrm{A}}\right|}{\sqrt{-\left(N^{2}+\mathcal{R}_{\varpi}\right)}}$,

are stabilized by magnetic tension. It is easy to show that the modes grow faster when $\boldsymbol{k}$ is parallel to the $z$-axis $\left(\theta_{k}=0\right)$, i.e. velocity and magnetic field perturbations grow in direction perpendicular to the rotation axis. The stability criterion, $N^{2}+\mathcal{R}_{\varpi}>0$, can easily be interpreted according to the relative size of the buoyancy term, $N^{2}$, and the shear term, $\mathcal{R}_{\varpi}$. Several different regimes result (Fig. 2):

- Magneto-shear instability (MSI): $\mathcal{R}_{\varpi} \ll N^{2}$ and $-4 \Omega^{2}<$ $N^{2}+\mathcal{R}_{\varpi}<0$. All modes longer than $\lambda_{\text {crit }}$ are unstable albeit with a vanishing growth rate as their wavelength $\lambda$ approaches infinity. The growth rate peaks for

$\lambda_{\mathrm{MRI}} \equiv 2 \pi / k_{\mathrm{FGM}} \sim \sqrt{2} \lambda_{\text {crit }}$,

where the limit $|C| \ll 8$ is used to obtain the second expression. It is important to note that $\lambda_{\mathrm{MRI}}$, which scales with

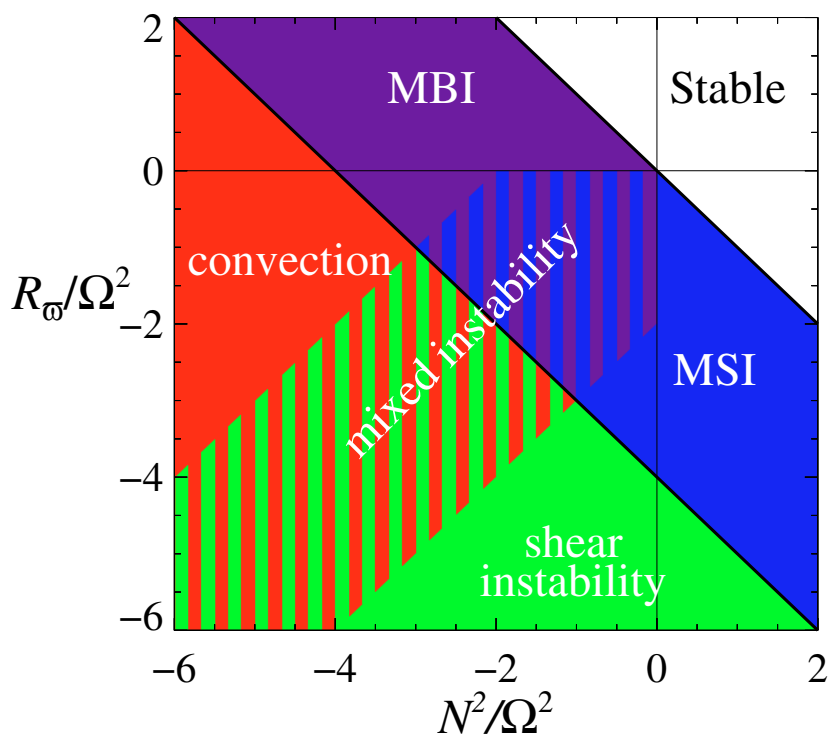

Fig. 2. Stability regions in the plane $\mathcal{R}_{\varpi} / \Omega^{2}$ vs $N^{2} / \Omega^{2}$. The solid thick line separating the stable region from the magneto-rotational instabilities (MSI and MBI) corresponds to $C=0$, and the solid thick line separating the magneto-rotational instabilities from the hydrodynamic instabilities (convection and shear instability) corresponds to the $C=-4$. The mixed zone is arbitrarily defined by $\left|\mathcal{R}_{\varpi} / \Omega^{2}-N^{2} / \Omega^{2}\right|<2$.

the background field strength $b_{0}$, becomes small for weak initial fields. Hence, in the limit of a pure shear instability, only relatively strong initial fields are accessible by numerical simulations due to the restrictive constraint on the grid size imposed by the requirement to resolve $\lambda_{\text {MRI }}$ by at least several grid zones;

- Magneto-buoyant instability $(M B I)^{2}: N^{2} \ll \mathcal{R}_{\varpi}$ and $-4 \Omega^{2}<$ $N^{2}+\mathcal{R}_{\varpi}<0$. This regime resembles the magneto-shear regime, but the instability is not driven by the shear, but rather by the unstable stratification;

- Magneto-convective instability: $N^{2} \ll \mathcal{R}_{\varpi}$ and $N^{2}+\mathcal{R}_{\varpi}<$ $-4 \Omega^{2}$. This regime corresponds to magnetized convective flow. The main difference is the stabilization of short modes $\left(\lambda<\lambda_{\text {crit }}\right)$ due to the magnetic tension. The more important the negative entropy gradient becomes with respect to the angular velocity gradient, the faster is the growth of infinitely long modes compared to the growth rate at $\lambda_{\mathrm{MRI}}$;

- Hydrodynamic shear instability: $\mathcal{R}_{\varpi} \ll N^{2}$ and $N^{2}+\mathcal{R}_{\varpi}<$ $-4 \Omega^{2}$. This case is not of interest in core collapse since for the differential rotation of PNS we always find $\mathcal{R}_{\varpi}>1.5 \Omega^{2}$.

Core collapse occurs in general in a mixed regime, where Alfvén modes and buoyant modes compete. Therefore, none of the above mentioned pure regimes holds for the MHD instabilities appearing during core collapse.

\section{Method}

\subsection{Code}

We use a newly developed three-dimensional Eulerian MHD code (Obergaulinger et al., in preparation) to solve the MHD equations, Eqs. (1)-(5). The code is based on a fluxconservative finite-volume formulation of the MHD equations

2 The reader should not confuse this instability with the magnetic buoyancy or Parker instability (Parker 1966), related to the magnetic field strength gradients. 
and the constrained-transport scheme to maintain a divergencefree magnetic field (Evans \& Hawley 1988). Using highresolution shock capturing methods (e.g., LeVeque 1992), it employs various optional high-order reconstruction algorithms including a total-variation diminishing piecewise-linear (TVD-PL) reconstruction of second-order accuracy, a fifthorder monotonicity-preserving (MP5) scheme (Suresh \& Huynh 1997), and a fourth-order weighted essentially non-oscillatory (WENO4) scheme (Levy et al. 2002), and approximate Riemann solvers based on the multi-stage (MUSTA) method (Toro \& Titarev 2006). The simulations reported here are performed with the MP5 scheme and a MUSTA solver based on the HLL Riemann solver (Harten 1983).

The simulations are performed using cylindrical coordinates, and include both three-dimensional and two-dimensional (i.e., axisymmetric) models. The computational grid covers a region of a few (typically one or two) kilometers aside resolved by at least 26 and at most 800 zones per dimension, corresponding to a resolution between 40 and $0.625 \mathrm{~m}$.

\subsection{Boundary conditions}

In local simulations, the choice of boundary conditions is a crucial issue, with possibly subtle effects on the flow geometry. The standard technique for local simulations of the MRI in accretion disks is the shearing-sheet method due to Hawley \& Balbus (1992). This approach consists of two important ingredients: (i) a transformation into a frame of reference co-rotating at the mean angular velocity of the shearing box, $\Omega_{0}$, and the linearizion of the rotation profile around $\Omega_{0}$; (ii) the use of shearing-sheet boundaries in the radial direction, and (in most cases) periodic boundary conditions in the perpendicular directions.

Periodic boundary conditions are often used in simulations of a small, representative sub-volume of a larger system. These boundary conditions are based on the idea that the entire system is covered by a homogeneous (e.g., cubic) lattice of identical sub-volumes. Consequently, the, e.g., left boundary of the simulated sub-volume is identified with the right boundary of an identical sub-volume translated by one lattice width.

A shearing box represents only a small part of the entire system. The influence of larger scales is considered by suitable boundary conditions, the most natural choice being periodic ones. These boundary conditions, however, do not allow one to impose global gradients throughout the shearing box, e.g., for differential rotation $\left(\partial_{\varpi} \Omega \neq 0\right)$. This shortcoming is eliminated by the linearization of the rotation profile and the transformation into the co-rotating frame since, in this case, the deviation from the background profile, $\delta \Omega$, is the dynamical variable rather than $\Omega$ itself. Thus, it is possible to use periodic boundary conditions in the radial direction accounting for differential rotation by an offset $\delta \phi(t)=t\left(\Omega_{\text {out }}-\Omega_{\text {inn }}\right.$ ), as described by (Hawley $\&$ Balbus 1992), where $\Omega_{\text {out,inn }}$ are the angular velocities at the outer and inner radial surface of the shearing box, respectively.

In contrast to accretion disks, thermodynamic variables in stars may have global gradients both in the direction perpendicular and parallel to the gradient of $\Omega$. Thus, standard shearingsheet boundaries cannot be used. Instead, we follow Klahr \& Bodenheimer (2003) and employ shearing-disc boundary conditions. We abandon the transformation into the co-rotating frame and assume radial periodicity of the deviation, $\delta q \equiv q-q_{0}$, of a variable $q$ from a given background distribution $q_{0}$, instead of periodicity of $q$ itself. We define the background distribution $q_{0}$ by its distribution at the initial time $t=0$, i.e. $q_{0} \equiv q(\varpi ; t=0)$. This recipe is applied to density, momentum, and entropy. As
Klahr \& Bodenheimer (2003), we observe the development of resonant radial oscillations which are suppressed, however, by damping the radial velocity in the first $n$ (we use $n=2$ ) computational zones at both radial boundaries. We point out that shearing-disc simulations allow for large-scale modifications of global gradients. In particular, angular momentum transport may modify the global rotation profile, and change the angular momentum and rotational energy of the matter in the computational volume. This process can eliminate the differential rotation causing the instability, and thus, terminate the growth of the MRI.

As we will show later, the evolution of our models depends crucially on whether we do or do not apply this damping term. However, we note here that the artificial oscillations prevented by the damping do not have a strong influence on the evolution of the MRI. We use, if at all, a damping of $v_{\varpi}$ by $1.25 \%$ in the innermost and outermost two zones of the grid, which is a considerably weaker damping than in the simulations of Klahr \& Bodenheimer (2003). Despite its relative weakness, the damping term is able to suppress weak radial motions across the boundary. Thus, it introduces a preferred length scale (the radial size of the box) into the otherwise shear-periodic simulation. Comparing simulations with and without damping (we will refer to these boundary conditions by $d$ and $p$, respectively), we can study the influence of a preferred scale on the MRI.

The box size of standard shearing-sheet simulations does not define a preferred length scale, i.e., these simulations are scale free and entirely local. In shearing-disc simulations, in contrast, the scale height of the thermodynamic variables introduces a physical length scale into the simulation. If this preferred length scale is smaller than the entire size of the star or disk, the simulations can be characterized as being semi-global.

The semi-global approach falls in between a purely local and a global one sharing merits and drawbacks with both methods. Similar to local simulations, semi-global simulations allow one to resolve a small part of the entire system better. Because they rely on a fixed lab frame and do not eliminate the mean rotation, the basic time scales are the same as in a global simulation of the same resolution. In a Keplerian disk dominated by rotation this might add a major difficulty to the numerical treatment of the problem. On the other hand, with pressure dominating over kinetic energy, the time step of our simulations is governed by the sound speed rather than the rotational velocity. As there is no way of eliminating the sound speed, we do not feel a need to use a shearing-sheet transformation.

We expect the MRI in core collapse to grow and reach saturation within several tens of milliseconds. The time step $\delta t \lesssim \delta x / c_{\mathrm{S}}$, on the other hand, is much smaller because of the high value of the sound speed in a post-collapse core $\left(c_{\mathrm{S}} \sim\right.$ $10^{10} \mathrm{~cm} \mathrm{~s}^{-1}$ ), where $\delta x$ is the width of the computational zone. Thus, we have to perform a large number (typically several millions) of time steps, which implies a limit on the grid resolution we can afford in the simulations, although the resolution is still much better than that of a global simulation.

\subsection{Initial conditions}

We use equilibrium initial models based on post-bounce cores from Obergaulinger et al. (2006b). Several tens of milliseconds after the core bounce, the shock wave has reached distances of a few hundred kilometers, the post-shock region exhibiting a series of damped oscillations as the proto-neutron star relaxes into a nearly hydrostatic configuration. We extract the radial profile of the gravitational potential along the equator of their 


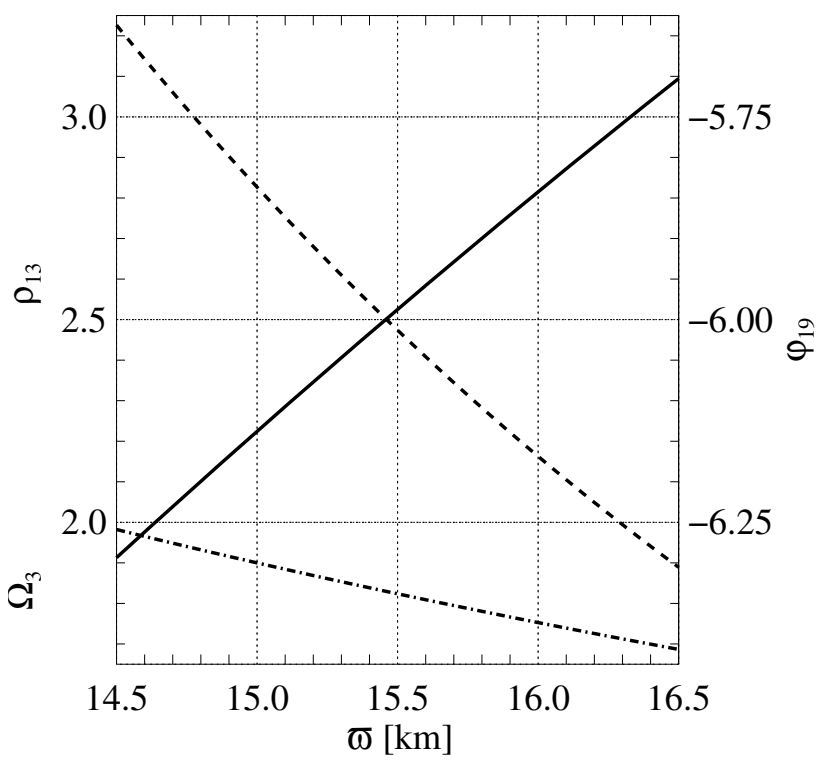

Fig. 3. Hydrostatic structure of the initial models. The diagram shows the gravitational potential $\varphi_{19}=\varphi /\left(10^{19} \mathrm{erg} \mathrm{cm}^{-3}\right)$ (solid lines, right $y$ axis), the density $\rho_{13}=\rho /\left(10^{13} \mathrm{~g} \mathrm{~cm}^{-3}\right)$ (dashed line, left $y$ axis), and the angular velocity $\Omega_{3}=\Omega /\left(10^{3} \mathrm{~s}^{-1}\right)$ (dash-dotted line, left $y$ axis). The entropy profile of this specific model is assumed to be flat.

model A1B3G $3^{3}$, and construct from that the density stratification within our shearing box solving the equation of hydrostatic equilibrium

$0=\rho \partial_{\varpi} \varphi-\partial_{\varpi} P+\varpi \rho \Omega^{2}$

for a given rotation profile

$\Omega(\varpi)=\Omega_{0} \varpi^{\alpha_{\Omega}}$,

where $\Omega_{0}$ and $\alpha_{\Omega}$ are constants. The pressure is determined using the hybrid equation of state in the form

$P=(S+1) \rho^{\gamma}$,

assuming an entropy profile of the form

$S(\varpi)=S_{0}+S_{1}\left(\varpi-\varpi_{0}\right)$

with constants $S_{0}$ and $S_{1}$. Equation (34) is solved in a radial domain of size, $\Delta \varpi$, which is either one or two kilometers large, centered at $\varpi_{0}=15.5 \mathrm{~km}$. The structure of an initial model, characterized by the set of parameters $\Omega_{0}=1900 \mathrm{~s}^{-1}, \alpha_{\Omega}=$ $-1.25, S_{0}=0$, and $S_{1}=0$, is shown in Fig. 3. This model has a radial density scale height of $H_{\rho}=\frac{P}{\partial_{\pi} P} \approx 3.8 \mathrm{~km}$, i.e., our computational grid covers a significant fraction of a density scale height. The rotation rate of $\sim 2000 \mathrm{~s}^{-1}$ corresponds to that of a rapidly rotating proto-neutron star with a rotational period of $\sim 3 \mathrm{~ms}$.

Assuming that the background gravitational potential is a function of $\varpi$ only, we construct cylindrically symmetric initial models. This approximation is justified by the small size of the simulation box in $z$-direction $(1 \mathrm{~km})$ compared to its radial position $(15 \mathrm{~km})$.

We added three different types of initial magnetic fields to the initial hydrostatic model:

[Model $U Z$ :] a uniform B-field in $z$-direction, $\boldsymbol{b}=\left(0,0, b_{0}^{z}\right)^{T}$.

3 This model experiences a core collapse that is halted by the stiffening of the equation of state above nuclear matter density.
[Model VZ:] a B-field in $z$ direction with vanishing net flux, $\boldsymbol{b}=$ $\left(0,0, b_{0}^{z} \sin \left(2 \pi\left(\varpi-\varpi_{0}\right) / \Delta \varpi\right)\right)^{T}$.

In all models, the initial field is weak, both in comparison with the thermal and the rotational energy of the models. The weakness of the associated Lorentz force justifies the use of hydrostatic instead of hydromagnetic equilibria as initial conditions.

From Eq. (33) and the values of typical model parameters we obtain the following estimate for the MRI wavelength

$\lambda_{\mathrm{FGM}} \sim 6.9 \mathrm{~km}\left(\frac{b}{10^{15} \mathrm{G}}\right)\left(\frac{\rho}{2.510^{13} \mathrm{~g} \mathrm{~cm}^{-3}}\right)^{-\frac{1}{2}}\left(\frac{\Omega}{1900 \mathrm{~s}^{-1}}\right)^{-1}$.

To properly simulate the evolution of the MRI, $\lambda_{\text {MRI }}$ should be resolved by at least a few grid zones. Using a grid resolution of $10 \mathrm{~m}$ or $20 \mathrm{~m}$, we thus can follow the growth of the MRI for magnetic fields exceeding several $10^{12} \mathrm{G}$ (Obergaulinger et al. 2006b,a). To trigger the instability, we impose a small random radial velocity perturbation with an amplitude of a few times $10^{-4 . .-2}$ of the rotational velocity.

\section{Results}

\subsection{General considerations}

In axisymmetry, the growth of the MRI requires a non-vanishing poloidal initial field. Axisymmetry restricts the dynamics of the MRI, suppressing a class of instabilities that affect the evolution of MRI-unstable modes (see below). Consequently, the predictive power of axisymmetric simulations for the evolution of the MRI is limited, and we cannot rely on them in determining the saturation amplitude of the instability in supernova cores. The growth of the instability does, however, not differ strongly from full 3D models. Thus, we can use 2D models to determine growth rates, while detailed conclusions can only be drawn from 3D models.

In axisymmetry, the flow is dominated by channel modes, a pattern of predominantly radial $^{4}$ flows of alternating direction stacked in $z$ direction (Balbus \& Hawley 1991). As the MRI grows, the channels start to merge and their characteristic length scales increase, but they survive as coherent flow structures throughout the entire evolution and, particularly, do not dissolve into turbulence.

The analysis of Goodman \& Xu (1994) shows that channel modes are an exact nonlinear solution of the axisymmetric MHD equations, which explains their stability observed in many numerical simulations. They are, on the other hand, unstable to genuinely 3D parasitic instabilities of, e.g., Kelvin-Helmholtz type. Consequently, in 3D, the channel modes appearing during the early growth phase of the MRI, do not persist until saturation. Instead, the channels decay due to the growing parasitic instabilities, and turbulence develops.

This basic picture emerged from many simulations of the MRI in accretion disks. As we will discuss in the following, our simulations confirm this result for the MRI in supernova cores.

\subsection{Axisymmetric models with no entropy gradient}

\subsubsection{Uniform initial magnetic fields}

Our models having no entropy gradient show the same dynamics as that observed in previous simulations of the MRI in accretion discs (see, e.g., Balbus \& Hawley 1998). We discuss first

\footnotetext{
4 In general, the channels are oriented parallel to the gradient of $\Omega$, wherever it points to.
} 


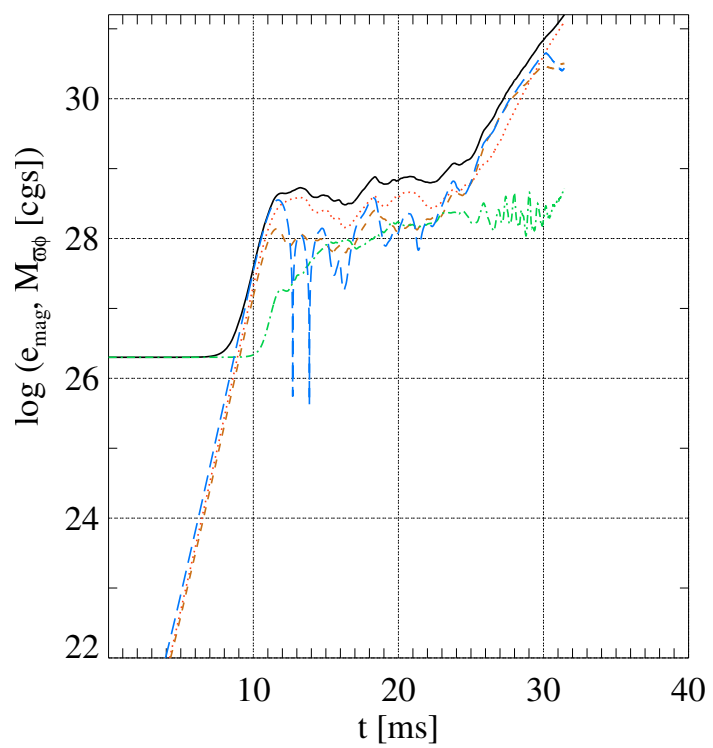

Fig. 4. Evolution of the mean magnetic energy density $e^{\text {mag }}$ (solid black line), the mean energy densities corresponding to the $\varpi$ (dotted red), $\phi$ (dashed brown), and $z$ (dash-dotted green) component of the magnetic field, and the absolute value of the mean Maxwell stress component $M_{\varpi, \phi}$ (dashed blue line) for an axisymmetric model with an initially uniform magnetic field $b_{0}^{z}=2 \times 10^{13} \mathrm{G}$ in z-direction, and a rotation law given by $\Omega_{0}=1900 \mathrm{~s}^{-1}$ and $\alpha_{\Omega}=-1.25$. The model was computed in a box of $L_{\varpi} \times L_{z}=1 \mathrm{~km} \times 1 \mathrm{~km}$ with a grid resolution of $5 \mathrm{~m}$.

the models with a uniform initial field $b_{0}^{z}$ in $z$-direction (model series UZ2) focusing on models with a rotational law given by $\Omega_{0}=1900 \mathrm{~s}^{-1}$ and $\alpha_{\Omega}=-1.25$ (see Eq. (35)). The evolution of these models is characterized by an exponential growth of the magnetic field, see e.g., Fig. 4 for a model with $b_{0}^{z}=2 \times 10^{13} \mathrm{G}$. The fastest growing MRI mode is well resolved in this model, and its growth rate $\sigma_{\mathrm{MRI}}=1.08 \mathrm{~ms}^{-1}$ is found to be close to the theoretical prediction $\sigma_{\mathrm{MRI}} \equiv \mathfrak{J}\left(\omega_{\mathrm{FGM}}\right) \approx\left|\alpha_{\Omega} \Omega_{0} / 2\right|=1.14 \mathrm{~ms}^{-1}$ (see Eq. (29)). The magnetic field reaches a maximum value of about $10^{15} \mathrm{G}$ at $t \approx 15 \mathrm{~ms}$, and the mean Maxwell stress component $M_{\varpi \phi}$ (see Eq. (13)) becomes large enough to alter the rotation profile considerably within a few tens of milliseconds. Consequently, the angular momentum of the gas drops drastically at $t \approx 25 \mathrm{~ms}$.

The growth of the MRI proceeds via channel flows, whose vertical extent and number depends on the initial magnetic field. Two typical channel flows are shown in Fig. 5. During the early phase of the instability ( $t=10.6 \mathrm{~ms}$; upper panel) eight distinct channels are present each one consisting of a pair of up- and down-flows in radial direction. The magnetic field is organized into eight elongated radial sheets, and this pattern is also imprinted onto the distribution of $\Omega$, as the magnetic field enforces co-rotation along field lines.

A flow topology dominated by channel modes implies a phase of exponential growth of the magnetic field, which ends when the channel modes are disrupted and a less organized, more turbulent state ensues (in Fig. 4 this happens at $t \approx 11 \mathrm{~ms}$ ). In most axisymmetric models, the turbulent state is only of transient nature, because after some time coherent channel flows form again leading to a secondary phase of exponential growth (see Fig. 4 at $t \approx 23 \mathrm{~ms}$ ).

Very late in the evolution $(t=30.7 \mathrm{~ms}$; lower panel of Fig. 5) we find only one large-scale channel flow which extends across the entire domain in radial direction. The magnetic field is now predominantly radial and is concentrated near
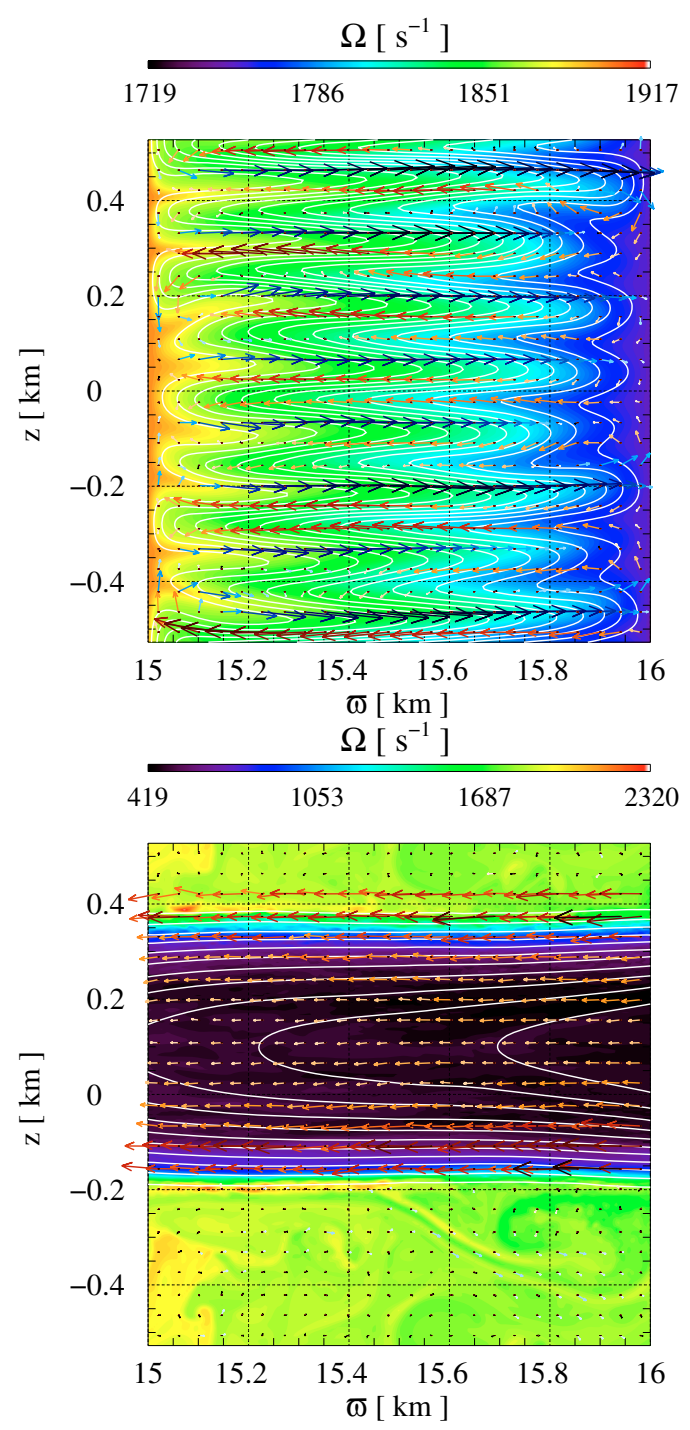

Fig. 5. The channel modes present in two snapshots taken from the model for which Fig. 4 shows the time evolution. The snapshots are taken at $t=10.6 \mathrm{~ms}$ (upper panel) and $t=30.7 \mathrm{~ms}$ (lower panel), respectively. The panels show the color coded angular velocity $\Omega$, the magnetic field lines (white), and the flow field. The colors of the velocity vectors indicate the magnitude and the direction of the flow: up- and down-flows are represented by blue and red vectors, respectively, their color intensity corresponding to the absolute value of the (poloidal) velocity (the darker the larger). The maximum velocities are $2.7 \times 10^{7} \mathrm{~cm} \mathrm{~s}^{-1}$ (upper panel), and $8.8 \times 10^{8} \mathrm{~cm} \mathrm{~s}^{-1}$ (lower panel), respectively.

the channel boundary. This coherent flow pattern is the result of a strong transport of mean angular momentum by Maxwell stresses. The stresses enforce co-rotation along field lines, and consequently turn the rotation profile, initially constant on cylinders $\varpi=$ const., by 90 degrees, so that $\Omega$ becomes a function of $z$ only. We can distinguish two regions of slow and fast rotation inside and outside $z \in[-0.15 ; 0.25]$, respectively. Inside the slowly rotating channel matter is accreting towards the center with $v_{\varpi} \sim 4 \times 10^{8} \mathrm{~cm}^{-1}$, while the rapidly rotating gas outside the channel has much slower, random velocities.

To investigate the dependence of the channel geometry on the initial magnetic field and the grid resolution we compute Fourier spectra of the radial component of the magnetic field, $b_{\varpi}$, for models with $\Omega_{0}=1900 \mathrm{~s}^{-1}$ and $\alpha_{\Omega}=-1.25$, and an initial field strength of 4,10 , and $20 \times 10^{12} \mathrm{G}$, respectively. The 


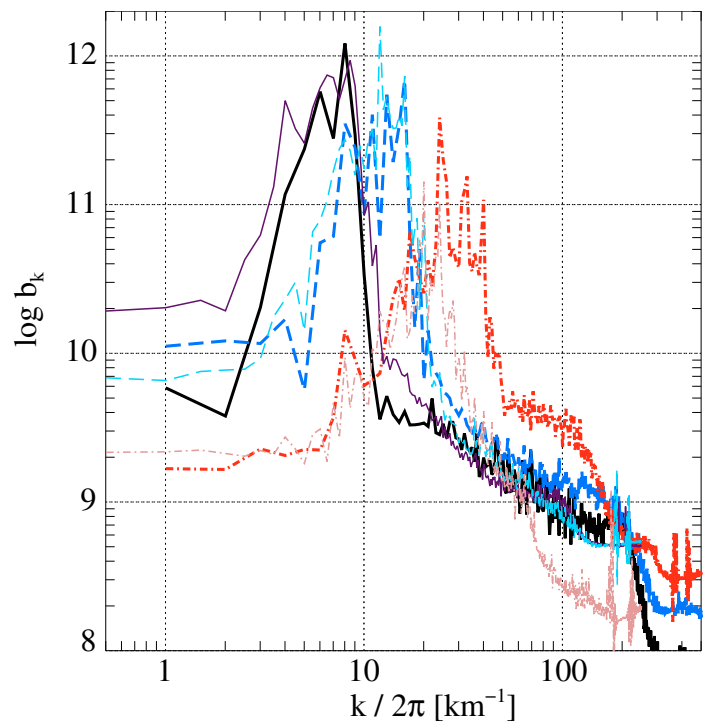

Fig. 6. Radially averaged Fourier spectra of the radial component of the magnetic field, $b_{\varpi}(z)$, for different uniform-field models at $t \approx 7.5 \mathrm{~ms}$. Models with $b_{0}=4 \times 10^{12} \mathrm{G}, 10^{12} \mathrm{G}$, and $2 \times 10^{13} \mathrm{G}$ are shown by the red dash-dotted, the blue dashed, and the black solid line, respectively. Thick and thin lines refer to a computational domain of $1 \mathrm{~km}^{2}$ and $2 \mathrm{~km}^{2}$, respectively. For all models a grid of $400^{2}$ zones is used.

simulations are performed in a box of either $1 \times 1 \mathrm{~km}^{2}$ or $2 \times 2 \mathrm{~km}^{2}$ using a $400^{2}$ grid (Fig. 6). At each radius we Fourier-transform $b_{\varpi}(z)$, and the resulting spectra $b_{\varpi}\left(k_{z}\right)$ (where $k_{z}$ is the vertical wave number) are then averaged over radius. We applied this procedure to the models during the growth phase of the instability at $t \approx 7.5 \mathrm{~ms}$. The first set of models $\left(1 \mathrm{~km}^{2}\right.$ domain, $2.5 \mathrm{~m}$ spatial resolution) exhibits growth rates close to the theoretical values, while this only partially holds for the models of the second set of models ( $4 \mathrm{~km}^{2}$ domain, $5 \mathrm{~m}$ spatial resolution). Due to insufficient spatial resolution the MRI in the model with the weakest initial field $\left(b_{0}^{z}=4 \times 10^{12} \mathrm{G}\right)$ grows slower than theoretically predicted. However, for the two more strongly magnetized models of this set $\left(b_{0}^{z}=10\right.$, and $\left.2 \times 10^{13} \mathrm{G}\right)$ the fastest growing modes are well resolved, and the MRI growth rates agree with the theoretical ones.

For each model the spectrum shows a distinct maximum corresponding to a dominant vertical length scale given by the width of one channel mode. The position of this maximum is a function of the initial magnetic field only, $k_{\max } \propto b_{0}^{-1}$, and thus does neither depend on the size of the computational domain nor on the resolution. A dependence on the last quantities is only observed, if the fastest growing mode is under-resolved. In this case, we recover the low- $k$ wing of the spectral peak, but find a truncated spectral distribution at higher wave numbers/smaller length scales.

MRI theory predicts that the growth rate is independent of the initial field strength. Neglecting magneto-convective modes, we can expect to observe this behavior in numerical simulations only if the grid is sufficiently fine to resolve the fastest growing modes close to $\lambda_{\mathrm{MRI}}$. Otherwise, if the grid is too coarse the growth rate should be much smaller. Our simulations reproduce this behavior. We show a comparison of the maximum growth rates from linear analysis $\left(\sigma_{\mathrm{FGM}}=\mathfrak{J}\left(\omega_{\mathrm{FGM}}\right) \approx\left|\frac{1}{2} \alpha_{\omega} \Omega_{0}\right|\right)$ and the numerical ones for models with different initial rotation laws $\left(\Omega_{0}\right.$ ranging from $950 \mathrm{~s}^{-1}$ to $1900 \mathrm{~s}^{-1}$, and $\alpha_{\Omega}$ from -1 to -1.25$)$ in Fig. 7. If $\lambda_{\text {MRI }}$ is under resolved for a given initial field $b_{0}$, the growth rate increases with $b_{0}$, but once the MRI wavelength is

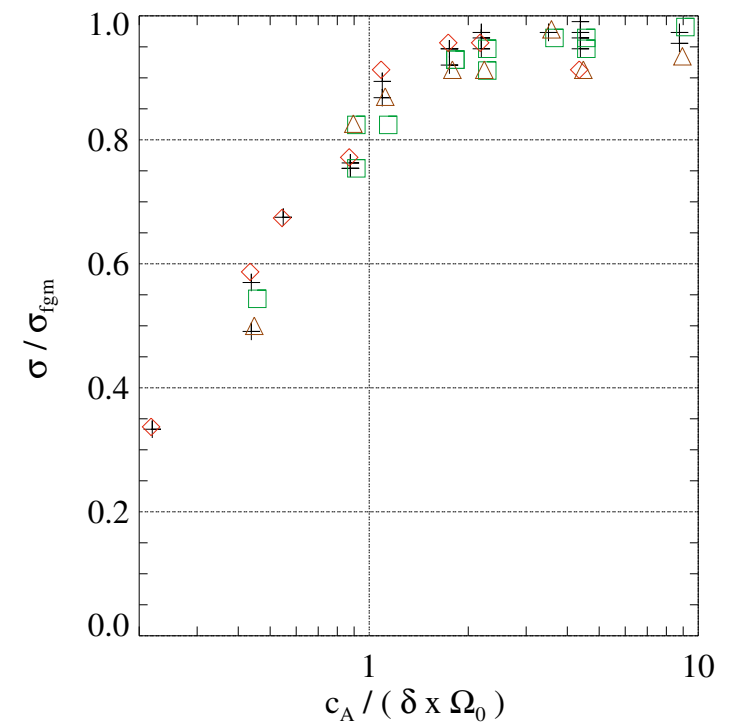

Fig. 7. Growth rate $\sigma$ of the MRI for axisymmetric models with uniform initial field as a function of the initial Alfvén speed normalized to the rotational velocity and the grid resolution, $c_{\mathrm{A}} /\left(\delta x \Omega_{0}\right)$. The colored symbols distinguish different initial rotational laws, where $\left(\Omega_{0}, \alpha_{\Omega}\right)$ are equal to $\left(1900 \mathrm{~s}^{-1},-1.25\right)$ [black plus signs], $\left(1900 \mathrm{~s}^{-1},-1\right)$ [red diamonds], $\left(950 \mathrm{~s}^{-1},-1.25\right)$ [green squares], and $\left(950 \mathrm{~s}^{-1},-1\right)$ [brown triangles], respectively.

well resolved, the growth rate becomes constant as theoretically predicted. Figure 7 implies the following criterion for a sufficient resolution of the MRI: $\Delta \varpi \gtrsim 2 \Omega_{0} / c_{\mathrm{A}}$. The growth rate of the instability does not depend on the size of the computational domain. For models with strong initial fields the computed MRI growth rate is smaller than $\sigma_{\mathrm{FGM}}$, because the MRI wavelength, i.e., the wavelength of the fastest growing mode, exceeds the box size. Thus, we can only properly simulate the slower growth of shorter modes.

\subsubsection{Channel disruption and MRI termination}

As long as the dynamics of the model is dominated by channel modes, the MRI grows exponentially. We observe a termination of its initial exponential growth - henceforth called MRI termination - as soon as the coherent channels are disrupted. Further MRI growth occurs after an eventual reformation of the channel flows. To understand these processes better, we study MRI termination in a large number of axisymmetric models with different initial magnetic fields, boxes of different size and grid resolution, and different boundary conditions.

Figure 8 shows the value of the mean Maxwell stress component $M_{\varpi, \phi}^{\text {term }}$ at MRI termination as a function of the initial magnetic field strength, $b_{0}$ for models with a rotational law $\Omega=1900 \mathrm{~s}^{-1} \varpi^{-1.25}$ and a vanishing entropy gradient. We can distinguish two classes of models according to the boundary conditions applied in the simulations (see Sect.3.2), the qualitative difference between the models with and without velocity damping near the boundaries being quite remarkable given the weak damping we apply. In models with damping $M_{\varpi, \phi}^{\text {term }}$ grows with increasing initial field strength until it levels off at a grid size dependent value (colored bands in Fig. 8). For the same models, when simulated without damping, we find that $M_{\varpi, \phi}^{\text {term }} \propto$ $b_{0}^{16 / 7}$ (gray band in Fig. 8) independent of the grid size. The "outliers" in the upper part of the figure correspond to models 


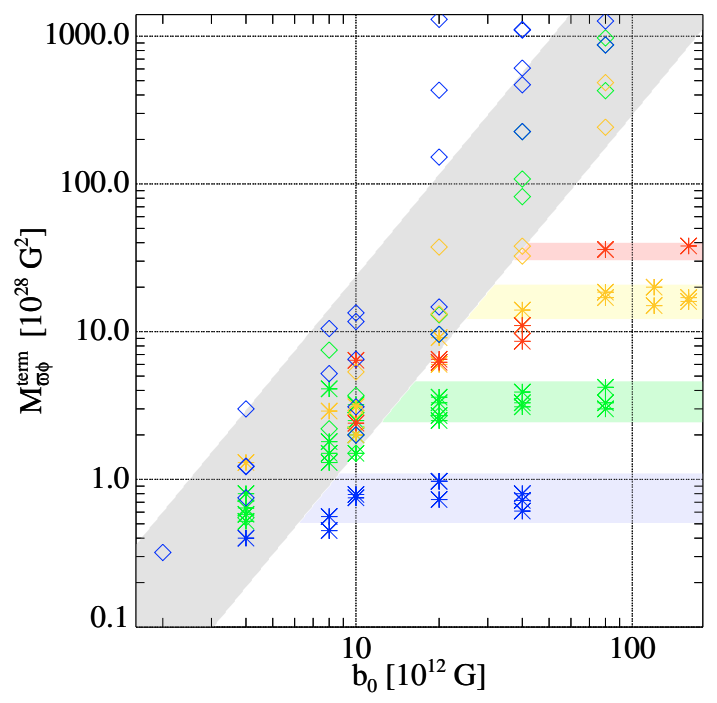

Fig. 8. Volume-averaged Maxwell stress component $M_{\varpi \phi}^{\text {term }}$ at MRI termination as a function of the initial magnetic field strength, $b_{0}$, for axisymmetric models with uniform initial magnetic field in $z$-direction, a rotational profile $\Omega=1900 \mathrm{~s}^{-1} \varpi^{-1.25}$, and a vanishing entropy gradient for a set of axisymmetric models. Blue, green, orange, and red symbols correspond to models computed in a square box having an edge size of $0.5,1,2$, and $4 \mathrm{~km}$, respectively. Models computed with and without velocity damping at the radial boundaries are denoted by asterisks and diamonds, respectively. The latter models show a box size independent scaling $M_{\varpi \phi}^{\text {term }} \sim\left(b_{0}^{z}\right)^{16 / 7}$ (gray band), while in models with damping $M_{\varpi \phi}^{\text {term }}$ saturates at high field strengths the saturation value depending on the box size (colored horizontal bands).

computed with a higher grid resolution than most other models. We will discuss this fact below.

To determine whether the radial or the vertical size of the computational grid is responsible for the leveling off of the Maxwell stress in the runs with radial damping we simulate two models with a grid of $0.5 \mathrm{~km} \times 2 \mathrm{~km}$ (for short called high models in the following), and $2 \mathrm{~km} \times 0.5 \mathrm{~km}$ (long models), respectively. Our results show that the determining factor for the growth is primarily the radial rather than the vertical box size, as both models follow the behavior of $M_{\varpi, \phi}$ as a function of $b_{0}$ for the respective radial grid sizes. The two classes of models also exhibit remarkably different post-growth dynamics. In the high models, a few channel modes reappear from the turbulent state, and a secondary phase of exponential growth of $M_{\varpi, \phi}$ sets in. Eventually, two of the newly formed channel modes merge. By this process, which occurs repeatedly, the number of channels decreases, and the final state of the flow is dominated by one short but wide channel mode. In the long models, on the other hand, no secondary exponential growth is observed, and the Maxwell stress remains approximately constant, albeit oscillating considerably due to the temporary presence of coherent flow patterns.

To interpret these results, one has to analyze the mechanism responsible for the disruption of the channel modes. We discuss this mechanism for an undamped model with $\Omega_{0}=$ $1900 \mathrm{~s}^{-1}, \alpha_{\Omega}=-1.25$, and an initial magnetic field strength $b_{0}=4 \times 10^{13} \mathrm{G}$ using a box of $0.5 \mathrm{~km} \times 0.5 \mathrm{~km}$ and a resolution of $100 \times 100$ zones. During the growth of the instability a few large channels are present, which are disrupted at MRI termination (at $\approx 15.9 \mathrm{~ms}$ ).

Figure 9 illustrates the disruption of one of the channel flows in some detail. At $t=15.850 \mathrm{~ms}$, the channel flow is still intact (left panel), and one recognizes two broad streams of inflowing and out-flowing gas both permeated by a strong radial magnetic field of opposite polarity. A broad current sheet separates the two flow regions. Owing to small-scale fluctuations in the flow, the field lines are not perfectly (anti-)parallel, and the current sheet is slightly deformed. These deformations act as seed perturbations for resistive instabilities of the tearingmode type. Although we evolve the equations of ideal MHD neglecting resistivity, the presence of numerical resistivity enables the growth of these instabilities, leading to a reconnection of anti-parallel field lines. As a consequence, the elongated current sheet dissolves into a configuration of $\mathrm{X}$ and $\mathrm{O}$ points (located at $\varpi \sim 15.25 \mathrm{~km}$ and $\sim 15.5 \mathrm{~km}$, respectively; see middle panel of Fig. 9). When field lines reconnect near the X point, the fluid is accelerated away from the reconnection point towards the $\mathrm{O}$ point. This causes the intense gas flow in positive radial direction at $(\varpi, z) \sim(15.35,0.1) \mathrm{km}$. The change of the topology of the magnetic field and of the flow continues shortly afterward (at $t=16.078 \mathrm{~ms}$; right panel of Fig. 9). The O point has grown in size, and the fluid is in vortical motion. As the vortex grows, field lines in the vortex are advected towards field lines of opposite polarity belonging to an adjacent channel flow (centered at $z \approx-0.15 \mathrm{~km}$ ), where reconnection occurs. Note the formation of a second $X$ point at $(\varpi, z) \approx(15.38,-0.03) \mathrm{km}$ (Fig. 9, right panel).

To demonstrate the growth of the tearing-mode instability and to support its importance for MRI termination, we compare the evolution of the mean Maxwell stress component $M_{\varpi \phi}$ and of the magnetic energy density of the $z$-component of the magnetic field, $e_{\text {mag }}^{z}$ of the model (see Fig. 10). Before the tearing mode grows $M_{\varpi \phi}$ and $e_{\text {mag }}^{z}$ increase, their growth rates being similar to that of the MRI. At $t=15.850 \mathrm{~ms}$, the growth rate of $e_{\mathrm{mag}}^{z}$ becomes larger than that of the MRI by one order of magnitude within less than $0.2 \mathrm{~ms}$, whereas $M_{\varpi \phi}$ approaches a maximum. Once the tearing mode is fully operative ( $t=15.983 \mathrm{~ms}$ ), the growth of $e_{\text {mag }}^{z}$ becomes slower but still continues due to the appearance of more tearing modes (see, e.g., the right panel of Fig. 10 at $(\varpi, z) \approx(15.38,-0.03) \mathrm{km})$. Subsequently, $e_{\text {mag }}^{z}$ begins to decrease as the tearing modes saturate.

According to the previous discussion the dynamics of the channel flows is dominated by the interplay between their growth due to the MRI and their destruction by resistive instabilities. Channel flows are unstable against tearing-mode-type instabilities at any point in their evolution. To study these instabilities in more detail, we have performed a set of simulations (see Appendix A) using simplified models of channel flows. We recapitulate our results, summarized in Eq. (A.11), here:

$\sigma_{\mathrm{r}} \propto\left(c_{\mathrm{A}}\right)^{7 / 4}\left(c_{\mathrm{S}}\right)^{-3 / 4}(a)^{-2}(\delta x)^{1}$

where $\sigma_{\mathrm{r}}, c_{\mathrm{A}}, c_{\mathrm{S}}, a$, and $\delta x$ are the growth rate of the instability, the Alfvén velocity corresponding to the channel magnetic field, the sound speed, the width of the channel, and the grid resolution, respectively. because the instability is not based on a physical resistivity, but is of purely numerical origin, no physical transport coefficient appears in Eq. (39). However, our results can be interpreted in terms of an effective resistivity $c_{\mathrm{A}} \delta x$, as detailed in Appendix A. In our models the width of the channel flow, $a$, is set by the MRI wavelength corresponding to the initial vertical magnetic field:

$\lambda_{\mathrm{MRI}} \propto b_{0}^{z} / \sqrt{\mathcal{R}_{\varpi}} \propto b_{0}^{z} /\left(\sqrt{\alpha_{\Omega}} \Omega_{0}\right)$

(see Eqs. (32), (33), and (35)). The width remains constant during the growth, as only mergers of adjacent channels occurring as a result of resistive instabilities can change the field topology. 

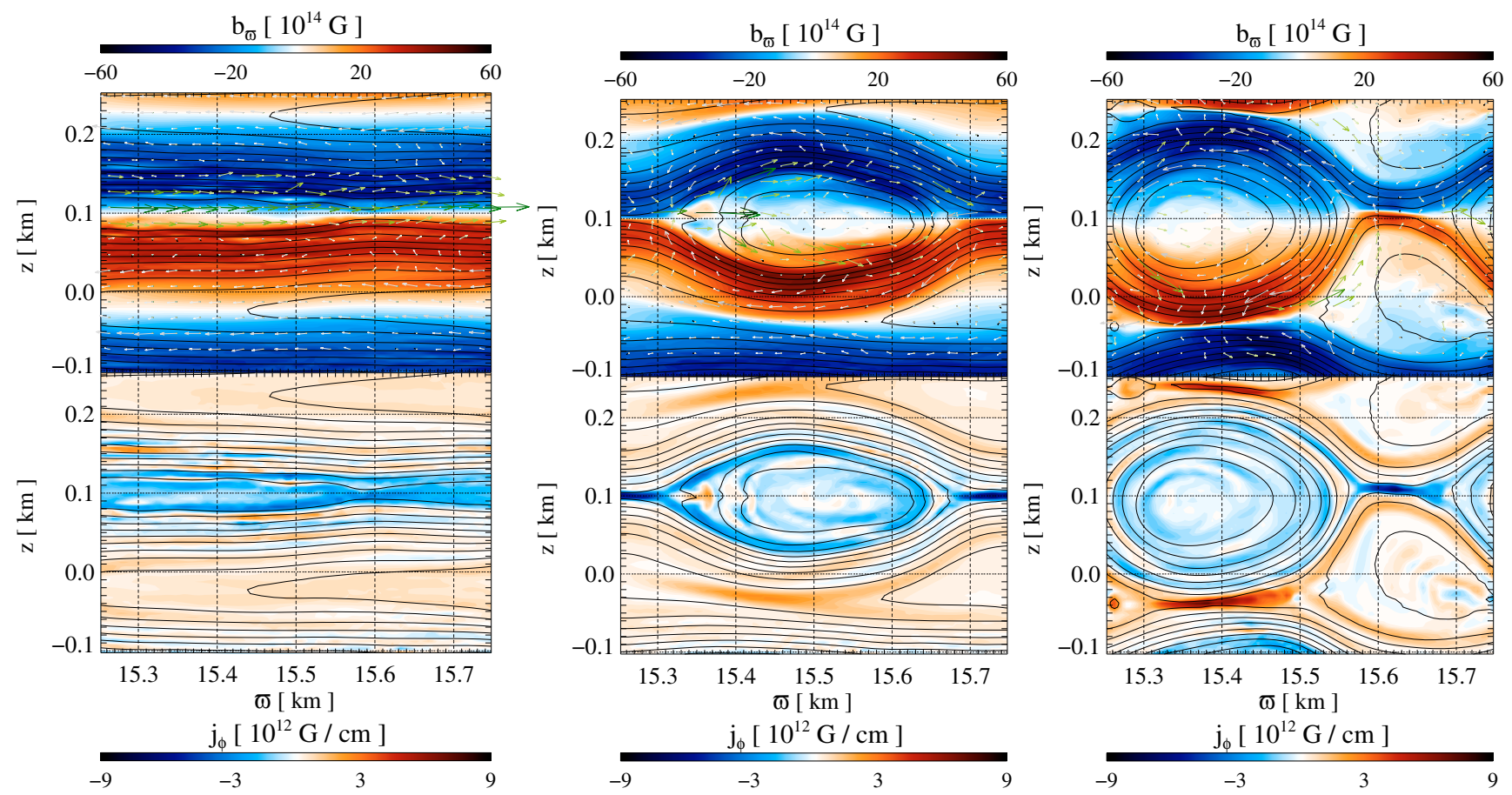

Fig. 9. The disruption of a channel mode in an axisymmetric uniform-field model. We show a section of a model with an initial field $b_{0}=4 \times 10^{13} \mathrm{G}$ computed on a grid of $0.5 \times 0.5 \mathrm{~km}^{2}$. The left, middle, and right panels display the color-coded radial component of the magnetic field $b_{\varpi}$ (top) and the current density $j_{\phi}=(\boldsymbol{\nabla} \times \boldsymbol{b})_{\phi}$ (bottom) before $(t=15.850 \mathrm{~ms})$, during $(t=15.983 \mathrm{~ms})$, and after $(t=16.078 \mathrm{~ms})$ the violent disruption of the channel flow, respectively. Additionally, magnetic field lines (black lines), and the velocity field (arrows; top only) are shown. The arrows are color-coded according to the magnitude and direction of the flow. Inflows and outflows are shown by gray and green vectors, respectively. The longest vector corresponds to a velocity of $|v|=7 \times 10^{8} \mathrm{~cm} / \mathrm{s}$.

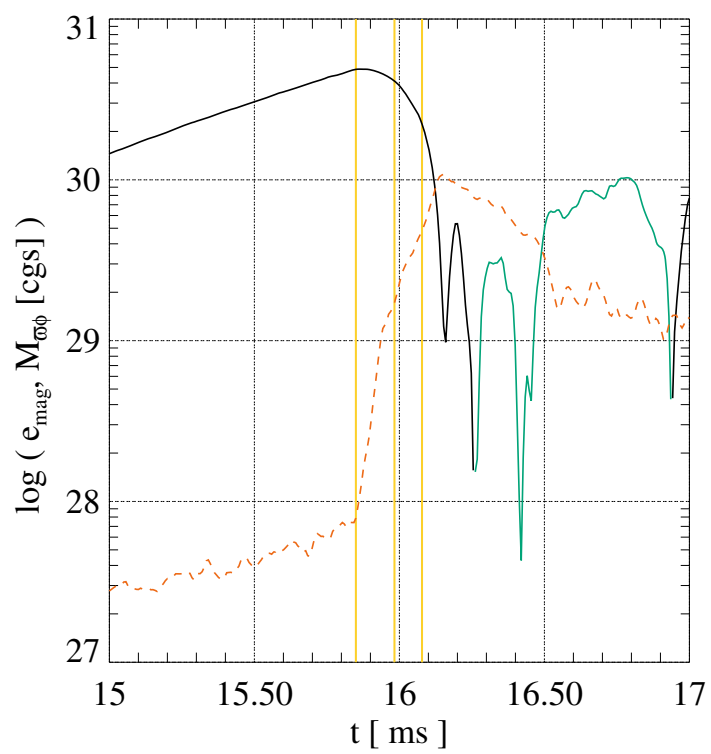

Fig. 10. Temporal evolution of the absolute value of the mean Maxwell stress component $M_{\varpi \phi}$ (solid line; the line is colored black where $M_{\varpi \phi}<0$, and green otherwise), and of the magnetic energy density of the z-component of the magnetic field, $e_{\mathrm{mag}}^{z}$ (dashed red line) of the model shown in Fig. 9. The vertical yellow lines mark the times of the snapshots shown in Fig. 9.

Our basic proposition for MRI termination is that channel flows are disrupted once the growth rate of the resistive instability exceeds the MRI growth rate:

$\sigma_{\mathrm{r}}>\sigma_{\mathrm{MRI}} \Rightarrow$ MRI termination.
Using in addition the functional dependence of $\sigma_{\mathrm{r}}$ (Eq. (39)), we can establish scaling laws for MRI termination for a given hydrodynamic background model. As the channel width scales with the MRI wavelength, $a \propto \lambda_{\mathrm{MRI}}$, and as the MRI growth rate is given by $\sigma_{\mathrm{MRI}} \equiv \mathfrak{J}\left(\omega_{\mathrm{FGM}}\right) \propto \alpha_{\Omega} \Omega_{0}$ (see Eq. (29)), we find for the Alfvén speed at MRI termination

$c_{\mathrm{A}}^{\mathrm{term}} \propto\left(c_{\mathrm{S}}\right)^{3 / 7}\left(b_{0}^{z}\right)^{8 / 7}\left(\Omega_{0}\right)^{-4 / 7}(\delta x)^{-4 / 7}$,

and for the corresponding Maxwell stress

$M_{\varpi \phi}^{\text {term }} \propto\left(c_{\mathrm{S}}\right)^{6 / 7}\left(b_{0}^{z}\right)^{16 / 7}\left(\Omega_{0}\right)^{-8 / 7}(\delta x)^{-8 / 7}$.

The latter equation implies that $M_{\varpi \phi}^{\text {term }}$ decreases with faster rotation. Two effects play a role in explaining this behavior. Firstly, slower rotation leads to slower MRI growth $\left(\sigma_{\mathrm{MRI}} \propto \Omega_{0}\right)$, and hence weaker magnetic fields are required for the tearing modes to overcome the MRI growth. Secondly, slower rotation implies wider channel flows $\left(a \propto\left(\Omega_{0}\right)^{-1}\right)$, i.e., resistive instabilities grow slower as $\sigma_{r} \propto a^{-2} \propto\left(\Omega_{0}\right)^{2}$ (see Eq. (39)).

The qualitative features of these scaling relations are:

1. stronger initial vertical fields and correspondingly wider channels tend to suppress resistive instabilities. Consequently, MRI termination requires more strongly magnetized channel flows;

2. finer grid resolution implies less numerical viscosity, and hence larger values for $c_{\mathrm{A}}^{\text {term }}$ and $M_{\varpi \phi}^{\text {term}}$;

3. the scaling of $M_{\varpi \phi}^{\text {term }}$ with the sound speed implies a proportionality between the Maxwell stress and the background pressure: $P \propto c_{\mathrm{S}}^{2}$, and thus $M_{\varpi \phi}^{\text {term }} \propto P^{3 / 7}$. This scaling is reminiscent of the $\alpha$-law in accretion discs according to which the (MRI-generated) viscosity is proportional to the gas pressure. 

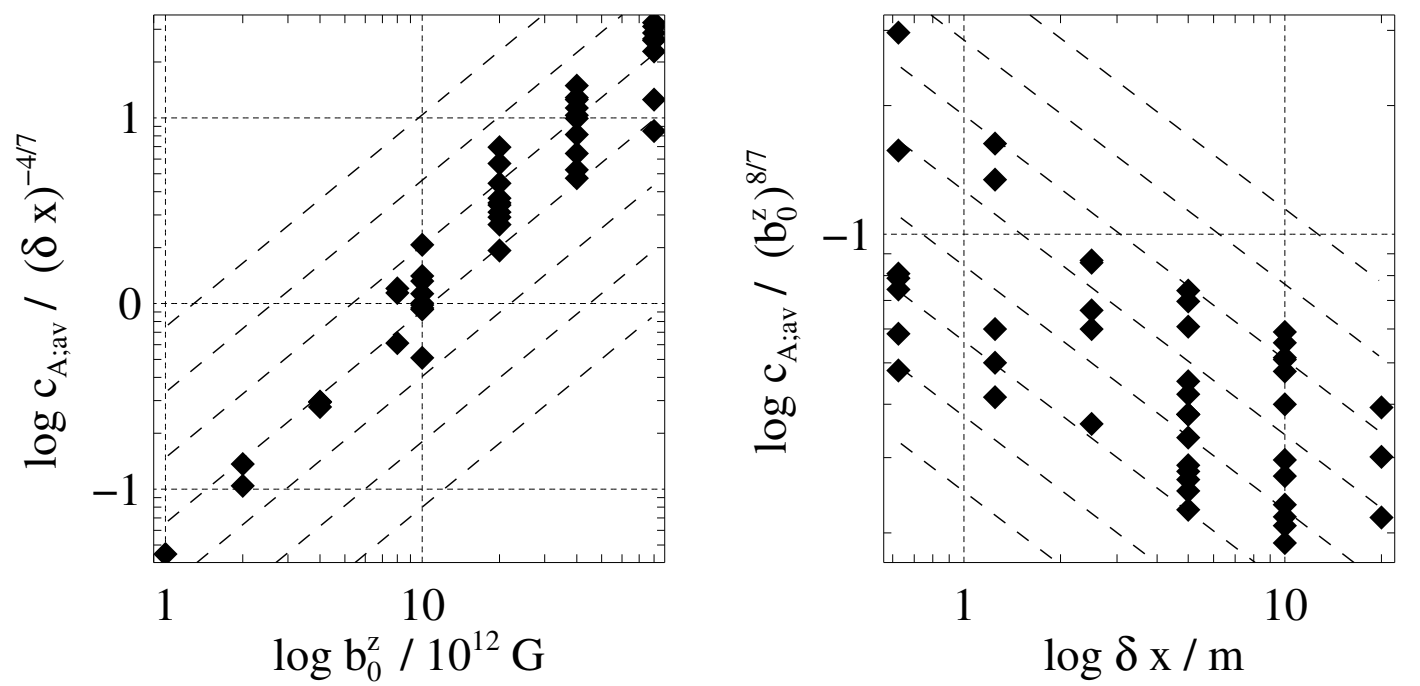

Fig. 11. Average Alfvén velocity - of models with uniform initial magnetic field and without velocity damping near the radial boundaries corresponding to the radial magnetic field at MRI termination normalized to $(\delta x)^{-4 / 7}($ left $)$ and $\left(b_{0}^{z}\right)^{8 / 7}$ (right) as a function of the initial magnetic field strength, $b_{0}^{z}$ (left), and the grid resolution $\delta x$ (right). The dashed lines represent the power laws expected from our analysis of resistive instabilities (see Eq. (42)). Note that we only consider well-resolved models $\left(\sigma_{\mathrm{MRI}} \geq 0.95 \mathrm{~ms}^{-1}\right)$ here.

Figure 11 shows that the average Alfvén velocity corresponding to the radial $^{5}$ magnetic field at MRI termination is well described by the scaling law given in Eq. (42). Similarly, Fig. 8 and Fig. 12 (upper panel) confirm that the data for $M_{\varpi \phi}^{\text {term }}$ obey the corresponding scaling law (Eq. (43)), too. The upper panel of the latter figure shows $M_{\varpi \phi}^{\text {term }}$ as a function of the initial magnetic field strength for models with different initial rotational laws. Obviously, the proportionality $M_{\varpi \phi}^{\text {term }} \propto\left(b_{0}^{z}\right)^{16 / 7}$ (light gray lines) provides a good approximation to the behavior of the models. Due to the small number of models the results should be taken with care, but a strong anti-correlation of $M_{\varpi \phi}^{\text {term }}$ with $\Omega_{0}$ is suggested. The data also do not support any dependence of $M_{\varpi \phi}^{\text {term }}$ on $\alpha_{\Omega}$. Finally, in Fig. 8 we noticed earlier some outliers at large values of $M_{\varpi \phi}^{\text {term }}$ which correspond to models computed on a fine grid. However, considering that MRI termination depends on grid resolution, all models lie within a narrow band which corroborates our scaling laws and provides more evidence of the importance of resistive instabilities in understanding the MRI. Consequently, physical (instead of numerical) transport coefficients should be used in MRI simulations, which may give rise to different scaling laws considering the growth rate of tearing modes.

Models with velocity damping. In models with uniform initial magnetic fields where the radial velocity is damped near the inner and outer radial boundary, i.e., in models located in the horizontal bands in Fig. 8, MRI termination happens earlier than predicted by our scaling laws, and the Maxwell stresses saturate for strong initial magnetic fields, the saturation value of $M_{\varpi \phi}^{\text {term }}$ being smaller for slower rotation (see lower panel of Fig. 12). This is due to the reconnection instability occurring close to the radial boundaries well before the theoretically predicted time of MRI termination. This premature reconnection is caused by the field geometry: due to the suppressed motion across the inner and outer radial boundary field lines must bent

\footnotetext{
5 The radial field is typical for all three components. Thus the Alfvén velocity corresponding to the total magnetic field shows the same dependence.
}

there in z-direction. Consequently, field lines of opposite polarity approach each other much earlier than in models without velocity damping, and efficient reconnection ensues. In this case, the onset of reconnection is determined by the field geometry rather than by the initial field strength. With reconnection occurring in the bent flux sheets near the radial boundaries instead between parallel flow sheets in the bulk volume as for non-damping boundaries, the width of a flux sheet is less important in determining the resistive growth rates. Thus, a slower MRI growth and smaller Maxwell stresses are found for slower rotating models when velocity damping is imposed. Apart from the dependence of $M_{\varpi \phi}^{\text {term }}$ on $\Omega_{0}$, we also find a dependence on $\alpha_{\Omega}$. Both dependences together give rise to a monotone relation between the strong-field limit of $M_{\varpi \phi}^{\text {term }}$ and the MRI growth rate, $\sigma_{\text {MRI, }}$ which qualitatively agrees with the above reasoning.

Once the initial channel flows are disrupted and the field geometry is changed by reconnection, the mean magnetic and kinetic energies, and the absolute value of the Maxwell stresses begin to fluctuate strongly around roughly constant values (see the phase between $11 \mathrm{~ms}$ and $23 \mathrm{~ms}$ in Fig. 4). Subsequently, a second phase of exponential MRI growth is possible, exhibiting a similar dynamics but involving less channel flows than the previous growth phase. The reduced number of channels is probably due to the strong increase in the vertical magnetic field during the growth of the tearing modes. Similarly to their predecessors, the newly formed channels are also unstable against resistive instabilities, but due to their larger width their disruption requires much higher Alfvén velocities, i.e., the MRI can lead to much higher Maxwell stresses in the second generation of channels. In principle, this process of formation and merger of channels can continue until only one single channel flow remains covering the entire box. We note that in later growth phases, the radial velocity and the magnetic field strength are typically so large that damping at the radial boundaries, if applied, does not lead to early saturation.

\subsubsection{Models with non-uniform initial magnetic fields}

Models having a non-uniform initial magnetic field exhibit a different evolution (see also Balbus \& Hawley 1998). To study 


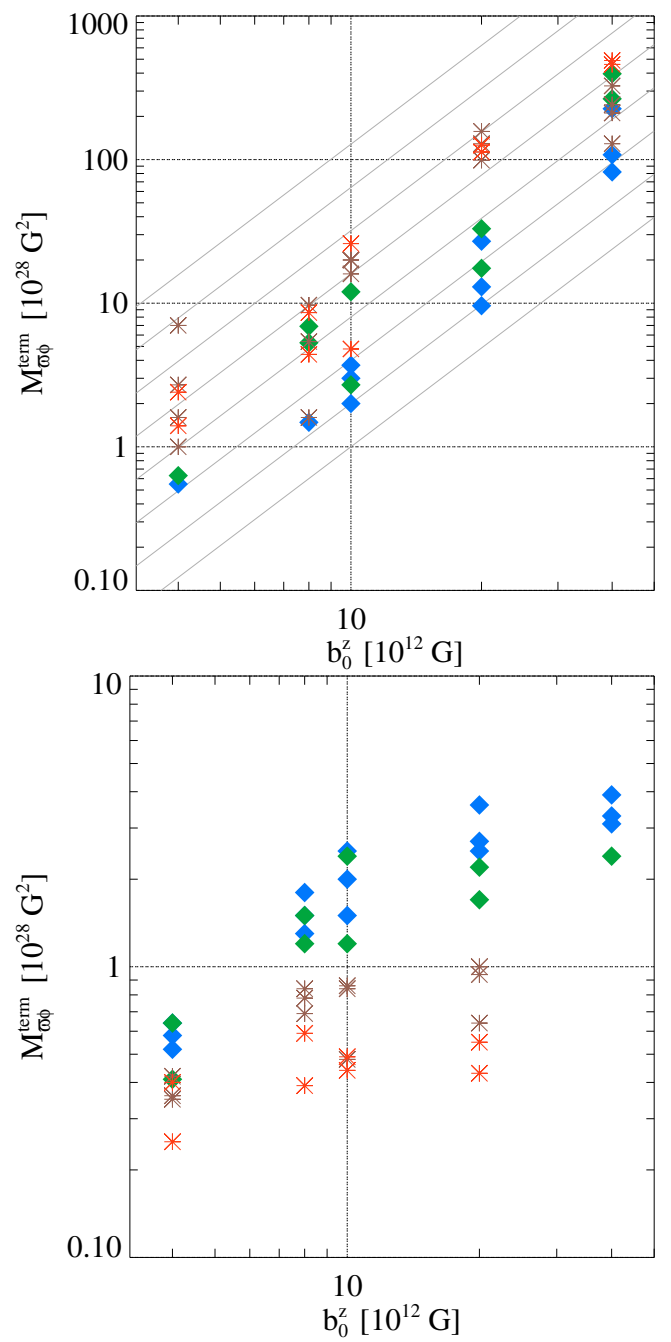

Fig. 12. Maxwell stress, $M_{\varpi \phi}^{\text {term }}$ at MRI termination as a function of the initial magnetic field strength, $b_{0}^{z}$ for models with different initial rotational profiles. The upper and lower panels show models with nondamping and damping boundary condition, respectively. The colored symbols distinguish different initial rotational laws where $\left(\Omega_{0}, \alpha_{\Omega}\right)$ are equal to $\left(1900 \mathrm{~s}^{-1},-1.25\right)$ [blue diamonds], $\left(1900 \mathrm{~s}^{-1},-1\right)$ [green diamonds], $\left(950 \mathrm{~s}^{-1},-1.25\right)$ [brown asterisks], and $\left(950 \mathrm{~s}^{-1},-1\right)$ [red asterisks], respectively. Note that only models with a box size $L_{\varpi} \times L_{z}=$ $1 \times 1 \mathrm{~km}^{2}$ and a resolution of 50,100, or 200 zones (per dimension) are considered here. The light gray lines in the upper panel illustrate power laws $\propto\left(b_{0}^{z}\right)^{16 / 7}$.

this evolution we simulated a set of models varying the initial magnetic field configuration and the boundary condition (applying velocity damping or not; see previous subsection). All models rotate initially according to the law given in Eq. (35) with $\Omega_{0}=1900 \mathrm{~s}^{-1}$ and $\alpha_{\Omega}=-1.25$.

We considered three types of non-uniform initial magnetic fields all of which have only a $z$-component. The first one

$b_{\mathrm{ZNF}}^{z}=b_{0}^{z} \sin \left(\frac{2 \pi \varpi}{\lambda_{\varpi}^{b}}\right) \times \frac{\varpi}{\varpi_{0}}$

varies sinusoidally with radius and scales in addition as $\propto \varpi$ to guarantee that the net magnetic flux through the surfaces of the computational box at $z=z_{0,1}$ vanishes. This is the standard zero flux field used in most MRI simulations. The second type of a non-uniform initial field considered by us is given by

$b_{\mathrm{Abs}}^{z}=\left|b_{\mathrm{ZNF}}^{z}\right|$.

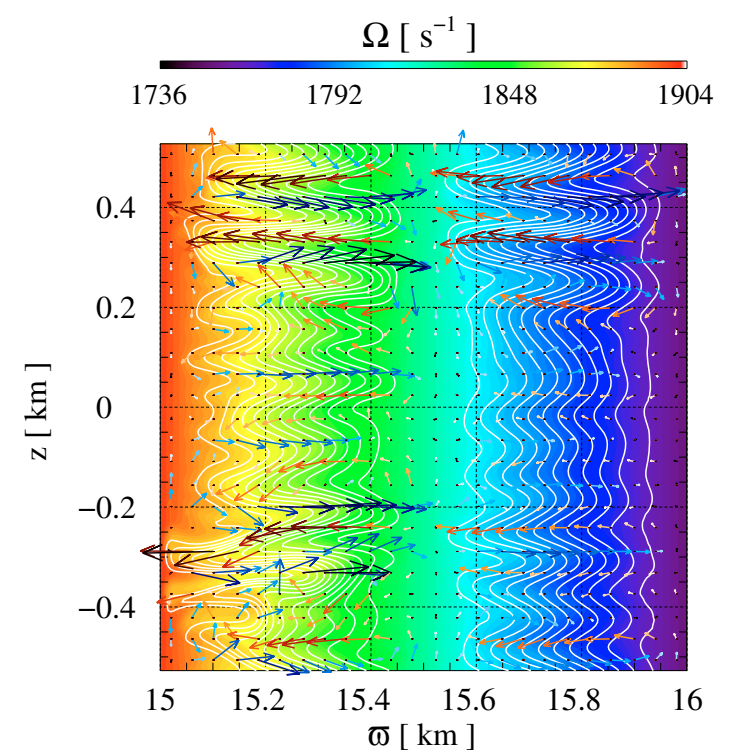

Fig. 13. An early state $(t=12.1 \mathrm{~ms})$ in the evolution of a model with $b_{0}^{z}=2 \times 10^{13} \mathrm{G}$ computed in a box of $L_{\varpi} \times L_{z}=1 \mathrm{~km}^{2}$ covered by $200 \times 200$ grid zones. Shown are the same variables as in Fig. 5. The maximum velocity is $1.1 \times 10^{7} \mathrm{~cm} \mathrm{~s}^{-1}$.

Finally, the third type also has a vanishing net magnetic flux, as $b_{\mathrm{ZNF}}^{z}$, but a step-like dependence on $\varpi$, i.e.,

$b_{\text {step }}^{z}=b_{0}^{z} \Theta\left(\varpi-\varpi_{\mathrm{c}}\right) \frac{\varpi}{\varpi_{0}}$,

where $\Theta$ and $\varpi_{c}$ denote the Heaviside step function and the radial coordinate of the center of the box, respectively.

For the first type of models the MRI starts growing via a multitude of channel modes giving rise to less amplification of the magnetic field than in models with a uniform initial field. Separate channels develop in the two radial regions of negative and positive $b^{z}$ (see Fig. 13), whereas the channels span the full radial extent if the initial field is uniform. The channel flows do not merge to form a few large-scale channels, but are destroyed by turbulence. After reaching a transient maximum, the magnetic energy and the Maxwell stress level off at values much less than for uniform-field models. The magnetic field becomes strongest right after MRI termination $\left(\sim 10^{15} \mathrm{G}\right)$. After $60 \mathrm{~ms}$ the maximum field strengths are about $2 \times 10^{14} \mathrm{G}$, and decrease to $10^{14} \mathrm{G}$ until the end of the simulation. Fields of this strength can change the $\Omega$ profile significantly only on time scales of many tens of milliseconds, i.e., at the end of the simulation the rotation profile is basically unchanged.

Figure 14 shows a comparison of the maximum Maxwell stress at MRI termination for the models with a non-uniform initial magnetic field. When velocity damping is applied the value of $M_{\varpi \phi}^{\text {term }}$ does not depend on the initial field geometry, and is determined by reconnection of anti-parallel field lines occurring close to the boundaries. If no velocity damping is applied, the evolution is similar to that of uniform field models, but depends on the field geometry. This finding can be understood in the light of our previous discussion of re-connective instabilities, and by the fact that $M_{\varpi \phi}^{\text {term }}$ is determined by reconnection in the bulk volume, and not by reconnection near the boundaries.

Models without velocity damping and the initial field, $b_{\mathrm{Abs}}^{z}$ develop large Maxwell stresses which increase with the initial field strength. The evolution of these models and the geometry of their channel flows are similar to those of models with uniform 


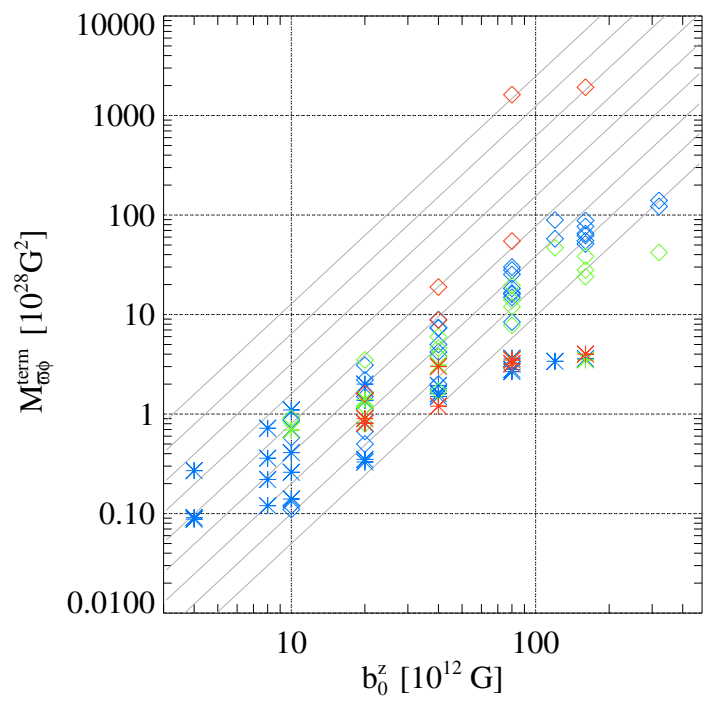

Fig. 14. Maxwell stress $M_{\varpi \phi}^{\text {term }}$ at MRI termination as a function of the initial magnetic field strength $b_{0}^{z}$ for models with non-uniform initial fields. Models with and without velocity damping are shown by asterisks and diamonds, respectively. The blue, green and red symbols denote models where the $z$-component of the initial magnetic field is given by $b_{\mathrm{ZNF}}^{z}$ (see Eq. (44)), $b_{\text {step }}^{z}$ (see Eq. (46)), and $b_{\mathrm{Abs}}^{z}$ (see Eq. (45)), respectively. The light gray lines illustrate power laws $\propto\left(b_{0}^{z}\right)^{16 / 7}$.

initial fields, i.e., the growth rates of tearing-modes are similar for both classes of models. For models in which the net magnetic flux vanishes initially we find that $M_{\varpi \phi}^{\text {term }}$ is roughly constant for sufficiently strong initial fields, the stress being slightly larger for sinusoidal ( $\left.b_{\mathrm{ZNF}}^{z}\right)$ than for step-like initial fields $\left(b_{\text {step }}^{z}\right)$. The models develop a more complex field morphology with more intense current sheets and more potential sites for reconnection than uniform field models. Thus, the growth rates of the resistive instabilities are comparable to those of the MRI for much weaker fields than in the uniform-field models. MRI termination also occurs at smaller values of $M_{\varpi \phi}^{\text {term }}$.

\subsection{Axisymmetric models with entropy gradients}

We also simulated some axisymmetric models imposing an additional entropy gradient. In this case, the instability criterion is more complicated and various instability regimes exist (see Sect. 2): (magnetic) shear instability, convection, and magnetobuoyant instability. Otherwise unstable modes may be stabilized by a stable thermal stratification or by fast (not necessarily differential) rotation. Tables B.1 (models with a positive entropy gradient) and B.2 (models with a negative entropy gradient) provide a list of the simulated models. Note that all models discussed in this subsection have a uniform initial magnetic field.

\subsubsection{Positive entropy gradients}

We first discuss differentially rotating models having a stabilizing entropy gradient comparing models with and without magnetic field. The non-magnetic models are stable due to the positive entropy gradient, i.e. initial perturbations do not grow.

The models with a magnetic field belong to the MSI regime (cf. Fig. 2), their MRI growth rates being reduced compared to models with no entropy gradient. We simulated models with different entropy gradients $\left(\partial_{\varpi} S=0.02,0.04,0.08 \mathrm{~km}^{-1}\right)$ and different adiabatic index of the equation of state $\left(\Gamma_{b}=1.31,5 / 3\right)$.
Generally, we find a good agreement between the analytic predictions and the numerical results. For $\partial_{\varpi} S<0.08$ the models are unstable belonging to the MSI regime, whereas an entropy gradient of $\partial_{\varpi} S=0.08$ suffices to stabilize the model. The growth rates agree well with the analytic ones, and the numerical models show the typical dependence of the growth rate of the MRI on the initial magnetic field strength. However, there exists one interesting difference: $\sigma$ increases from small values for weak initial fields for which $\lambda_{\mathrm{MRI}}$ is under resolved and converges to the correct growth rate for strong fields for which $\lambda_{\mathrm{MRI}}$ exceeds the grid resolution significantly. Unlike for models without entropy gradient, the growth rate becomes largest for magnetic fields for which the MRI wavelength is similar to the grid resolution, and at these field strengths the numerical growth rates can exceed the theoretical ones.

Dynamically the models behave similarly as models without an entropy gradient. Channel flows develop during the growth phase of the MRI, their width being set by the MRI wavelength. MRI termination occurs due to the growth of tearing-mode-like resistive instabilities. When velocity damping is applied, the maximum Maxwell stress at MRI termination, $M_{\varpi \phi}^{\text {term }}$, is determined by the box size. Comparing models with a positive entropy gradient and with no entropy gradient we find a common linear relation between $M_{\varpi \phi}^{\text {term }}$ and $\sigma_{\text {MRI }}$, indicating a common reason for MRI termination (see Fig. 15).

According to Eq. (28), the channels are wider in models with larger entropy gradients. As wider channels are less prone to resistive instabilities, they can support stronger fields before being disrupted (see discussion above), i.e. $M_{\varpi \phi}^{\text {term }}$ is larger in models with larger entropy gradients. The MRI growth rate, on the other hand, is smaller for larger entropy gradients (see Eq. (29)) implying that $M_{\varpi \phi}^{\text {term }}$ decreases with increasing entropy gradient. Both effects taken together suggest a weak anti-correlation of $M_{\varpi \phi}^{\text {term }}$ with the size of the (positive) entropy gradient. An anticorrelation is also suggested by our numerical results, although more models are needed to confirm it. It is unclear, for example, whether the growth rates of resistive instabilities derived in Sect. 4.2.2 also hold for stably stratified media, and whether the boundary conditions have an influence in models with large entropy gradients. Small perturbations of the quasi-periodic (because of global gradients) radial entropy distribution may leave their imprint on MRI termination by enforcing a preferred length scale, thus clouding effects due to a variation of $\partial_{\varpi} S$.

We have also simulated a few of the models using an idealgas equation of state, $P=(\Gamma-1) \varepsilon$, instead of the hybrid EOS finding, however, no effect on the evolution of the models.

\subsubsection{Negative entropy gradients}

Convection can develop in models having a negative entropy gradient, but it can be suppressed by rapid rigid or differential rotation. If a magnetic field is added to a convectively unstable system which cannot be stabilized by rotation, the system is located in the "convection" regime of Fig. 2. In a situation where rotation suffices to suppress the convective instability, the addition of a weak magnetic field puts the system into the "MBI" regime, and a magneto-buoyant instability similar to the standard (i.e., $\left.\partial_{\varpi} S=0\right)$ MRI develops. When $\partial_{\varpi} S<0$ the equivalent of the standard MRI corresponds to the "MSI" regime in Fig. 2.

Before discussing our results (see Table B.2 for a list of the simulated models), we need to comment on the boundary conditions. Allowing for radial transport of energy shearing-disc boundaries can, in principle, lead to a transport of entropy across 


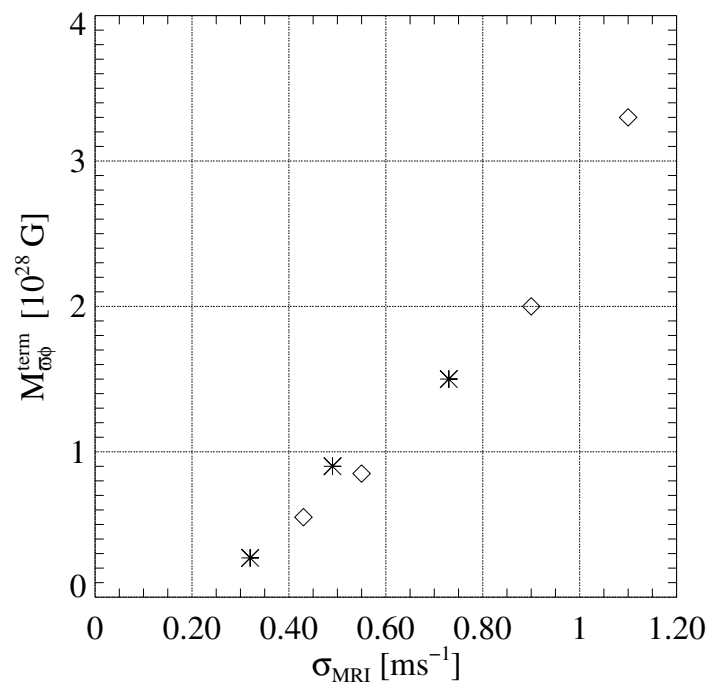

Fig. 15. Maxwell stress at MRI termination, $M_{\varpi \phi}^{\text {term }}$, as a function of the MRI growth rate, $\sigma_{\mathrm{MRI}}$, for models with zero (diamonds) and positive (plus signs) entropy gradients.

the pseudo-periodic boundaries, thus modifying the initial entropy profile. By comparing shearing-disc models and models with reflecting boundary conditions, we verified that none of these boundary conditions suppresses the growth of the MRI, and that both give similar results.

Let us first consider a non-magnetic model which rotates rigidly with an angular velocity $\Omega_{0}=1000 \mathrm{~s}^{-1}$ and has an entropy profile given by $S_{0}=0.2$ and $\partial_{\varpi} S=-0.075 \mathrm{~km}^{-1}$ (see Eq. (37)). With $N^{2} / \Omega_{0}^{2} \approx-14$ and $\mathcal{R}_{\varpi} / \Omega_{0}^{2}=0$, the model belongs to the convective regime. Buoyant modes are unstable and grow at a theoretical rate $\sigma_{\text {th }}=3.4 \mathrm{~ms}^{-1}$. Simulated on a grid with $2.5 \mathrm{~m}$ spatial resolution, the model is unstable with a numerical growth rate $\sigma=2.7 \mathrm{~ms}^{-1}$, and convection sets in quickly. The flow is dominated by a few (one or two) fairly circular convective rolls. Due to the transport of entropy and angular momentum by the overturning fluid, the model develops complementary entropy and rotation profiles characterized by "cold" (i.e., low-entropy), rapidly rotating matter in down-flows and "hot" (i.e., high-entropy), slowly rotating matter in up-flows. The redistribution of angular momentum and entropy leads to an average (with respect to the $z$-coordinate) rotational profile of the form $\Omega \propto \varpi^{-2}$, i.e., constant specific angular momentum (see Fig. 16), and a flat entropy profile.

For a faster rotation rate of $\Omega_{0}=1500 \mathrm{~s}^{-1}$, corresponding to $N^{2} / \Omega_{0}^{2} \approx-5.7$, i.e., still in the convective regime, the evolution is similar except for a reduced growth rate $\left(\sigma \approx 1.6 \mathrm{~ms}^{-1}\right)$ due to rotational stabilization. The model develops differential rotation with the same $\varpi$-dependence as in the case of slower rotation. If we increase the rotation rate to $\Omega_{0}=1900 \mathrm{~s}^{-1}\left(N^{2} / \Omega_{0}^{2} \approx-3.2\right)$ buoyant modes are stabilized by rotation.

The above results also hold if the initial model is rotating differentially. In particular, convection (i.e., the negative initial entropy gradient) gives also rise to a rotation law of the form $\Omega \propto \varpi^{-2}$, and a flat entropy profile.

Next, we add a magnetic field of $b_{0}^{z}=10^{13} \mathrm{G}$ to a convective model, i.e., to a model with a negative entropy gradient rotating too slowly (in our case, for rigid rotation, with $\Omega_{0} \lesssim 1500 \mathrm{~s}^{-1}$ ) for convection to be stabilised by rotation. The temporal evolution of the magnetic energy and the Maxwell stress of this model is shown in Fig. 17, while Fig. 18 gives the spatial distribution of the entropy of the model at two different times.

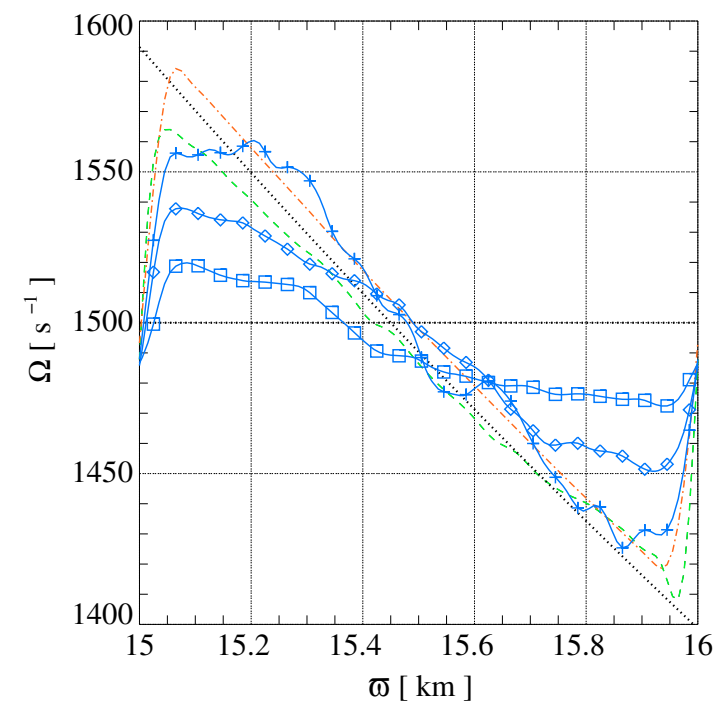

Fig. 16. $\Omega$, averaged over $z$, as a function of $\varpi$ for non-magnetic convective models at $t=51.9 \mathrm{~ms}$ (green dashed), with an initial magnetic field $b_{0}^{z}=10^{12} \mathrm{G}$ at $t=51.9 \mathrm{~ms}$ (red dash-dotted), and an initial field $b_{0}^{z}=10^{13} \mathrm{G}$ (blue solid lines), respectively. For the latter model the different symbols indicate different epochs: $t=11.5 \mathrm{~ms}$ (plus signs), $t=20.8 \mathrm{~ms}$ (diamonds), and $t=41.7 \mathrm{~ms}$ (squares). The dotted black lines show the initial rotation law $\left(\Omega_{0}=1500 \mathrm{~s}^{-1}\right.$, and a power-law profile $\Omega \propto \varpi^{-2}$.

Initial perturbations are amplified rapidly, but saturation sets in within 6 milliseconds the growth rate being slightly higher than in the non-magnetic models. With $N^{2} / \Omega_{0}^{2} \approx-5.7$, the model is dominated by buoyant modes, but there still exists some influence of the Alfvén modes. In particular, although infinitely long modes grow rapidly, the fastest growing modes are Alfvén modes of finite wavenumber which depends on $b_{0}^{z}$. For sufficiently strong initial fields (or sufficiently fine resolution), these modes are numerically resolved, and have a growth rate exceeding that of the corresponding non-magnetic model. The magnetic field strength increases exponentially as the instability develops, and at the onset of saturation large convective rolls develop (left panel of Fig. 18). In the saturation phase (right panel of Fig. 18) the flow geometry differs considerably from that of the corresponding non-magnetic model. It consists of down-drafts of cold material and up-flows of hot gas forming small-scale structures rather than large circular convective rolls. Like in the non-magnetic model, cold and hot regions correspond to regions of low and high angular velocity, respectively. Differential rotation with constant specific angular momentum, $\Omega \propto \varpi^{-2}$, develops due to hydrodynamic transport of angular momentum in convective overturns. The magnetic energy related to the radial component of the magnetic field and the Maxwell stress component $M_{\varpi \phi}$ remain high during saturation, i.e. angular momentum transport converts the $\varpi^{-2}$ rotation law prevailing at early epochs into nearly rigid rotation (Fig. 16).

If for the same initially rigidly rotating model the initial magnetic field is too weak to resolve $\lambda_{\text {MRI }}$ (we simulated two models with $b_{0}=10^{4}$ and $10^{12}$, respectively) convection develops. The growth rates are similar to those of non-magnetic models. The weakest initial field, $b_{0}^{z}=10^{4} \mathrm{G}$, has no impact at all, and the evolution of the model with an initial field of $10^{12} \mathrm{G}$ differs only slightly from that of the non-magnetic model. The magnetic field increases exponentially as the instability grows. Large persistent convective rolls form, and differential rotation develops. After an initial exponential growth the mean magnetic energy 


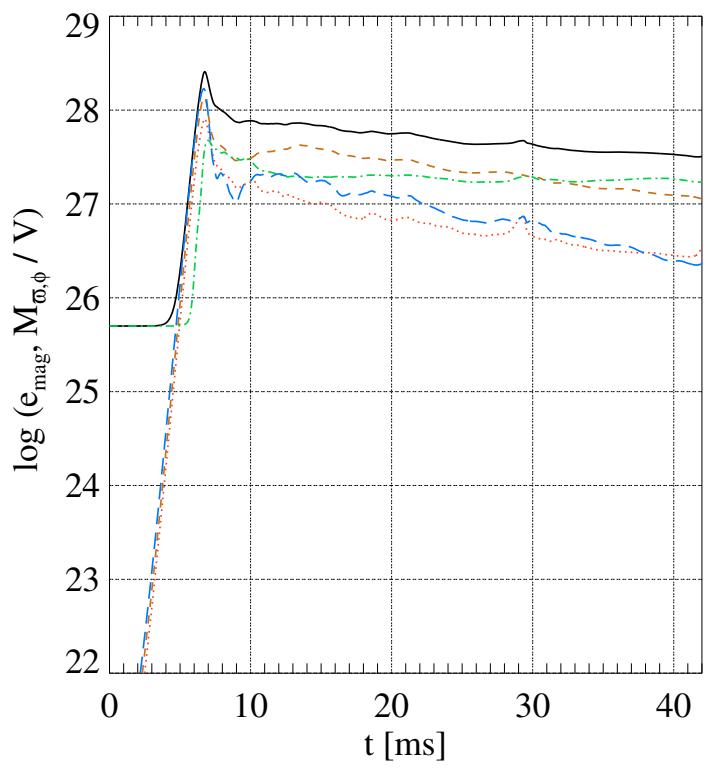

Fig. 17. Evolution of the mean magnetic energy density $\mathrm{e}^{\text {mag }}$ (solid black line), the mean energy densities corresponding to the $\varpi$ (dotted red), $\phi$ (dashed brown), and $z$ (dash-dotted green) component of the magnetic field, and the absolute value of the mean Maxwell stress component $M_{\varpi, \phi}$ (dashed blue line) for the model whose rotational profiles at different times are shown in Fig. 16, i.e., a rigidly rotating model $\left(\Omega_{0}=1500 \mathrm{~s}^{-1}\right)$ with an initial magnetic field of $b_{0}^{z}=10^{13} \mathrm{G}$.

remains large, but the contribution of the radial component of the magnetic field and the mean Maxwell stress component $M_{\varpi \phi}$ decrease almost by four and two orders of magnitude, respectively. Consequently, no significant angular momentum transport occurs due to magnetic stresses, and similar to the non-magnetized model a $\Omega \propto \varpi^{-2}$ rotation law develops. Fig. 16).

We now consider the $M B I$ regime (see Fig. 2) and discuss models rotating initially rigidly with $\Omega_{0}=1900 \mathrm{~s}^{-1}$ and having an initial entropy gradient $\partial_{\varpi} S=-0.1 \mathrm{~km}^{-1}$ (see Table B.2), which implies $N^{2} / \Omega_{0}^{2} \approx-3.6$. Without magnetic field, the instability of the buoyant modes is suppressed by the fast rotation. However, if a weak magnetic field is added, an instability of the MBI type develops, i.e., the Alfvén modes become unstable. The numerical growth rates show a similar dependence on the magnetic field strength as in case of the standard (i.e., $\partial_{\varpi} S=0$ ) MRI, because $\lambda_{\text {MRI }}$ is resolved. The instability grows rapidly ( $\sigma \sim 1.4 \mathrm{~ms}^{-1}$, similar to the theoretical value $\sigma_{\text {th }} \approx 1.7 \mathrm{~ms}^{-1}$ ). During the growth phase channel modes appear, which lead to a transport of both angular momentum and entropy. After an exponential initial growth and some decrease after MRI termination the mean magnetic energies contained in the total magnetic field and all three field components remain large (corresponding to field strengths of $\sim 10^{14} \mathrm{G}$ ), but the mean Maxwell stress component $M_{\varpi \phi}$ drops to zero within ten milliseconds oscillating afterward with decreasing amplitude between positive and negative values. Hence, large-scale angular momentum transport is limited. At the end of the simulation, the model shows considerable variations in $\Omega$, but there is no clear indication of a mean differential rotation of the form $\Omega=\Omega(\varpi)$. The entropy profile in the saturated state is almost flat.

Finally, we summarize a few common features of the models having a negative entropy gradient (see Table B.2). All models develop instabilities in accordance with the flow regime to be expected from their model parameters. The growth rates of all models are, within the uncertainties, similar to the theoretical predictions. As for the dynamics, we have to distinguish models in the convective regime from those in the mixed and MBI regimes. The former class of models shows convective mushrooms and large-scale overturns with only little influence of the magnetic field, whereas the last class is dominated by channel flows. Consequently, angular momentum transport by hydrodynamic flow leads to a rotational profile $\Omega=\varpi^{-2}$ for models in the convective regime, while models in the mixed-type regime tends towards rigid rotation the angular momentum transport being dominated by magnetic fields. Termination of the instability growth occurs for models both in the MBI and mixed-type regime analogously to that of models in the MSI regime without entropy gradient, i.e., by reconnection in resistive instabilities altering the topology of the channel flows. Consequently, we find similar dependencies on the initial field strength, the grid resolution, and the type of boundary conditions. The instability in convective models, on the other hand, saturates when the initial entropy gradient is removed by vigorous entropy transport due to overturning fluid motions.

\subsection{Three-dimensional models}

The results of the axisymmetric simulations discussed in the previous section demonstrate the possibility of MRI-driven field amplification in core collapse supernovae, and provide some insight into the evolution of MRI unstable layers in the core. However, to address the MRI problem in full generality, one has to consider three-dimensional models, because the assumption of axisymmetry implies severe restrictions for the dynamics of the magnetic and kinetic fields. The most important limitations are that, in axisymmetry, a toroidal field cannot be converted into a poloidal one, and that the disruption of the channel flows requires non-axisymmetric parasitic instabilities (Goodman \& Xu 1994).

As 3D simulations are computationally much more expensive than 2D ones, we could not perform a comprehensive study, but had to focus on a few selected models. We simulated models with different field geometries and varied the initial field strength, the entropy profile, and the grid size (see Tables B.3 and B.4).

\subsubsection{Uniform initial magnetic fields, no entropy gradient}

We first discuss models which have a uniform initial magnetic field $b_{0}^{z}$ in $z$-direction, no entropy gradient, and rotate differentially with $\Omega_{0}=1900 \mathrm{~s}^{-1}$ and $\alpha_{\Omega}=-1.25$ (see Eq. (35)). If the MRI wavelength is well resolved (e.g., for models with initial field strengths of $2 \times 10^{13} \mathrm{G}$ and $4 \times 10^{13} \mathrm{G}$ simulated at a grid resolution of $\delta \varpi=20 \mathrm{~m}$ ), the growth rate is high and independent of $b_{0}^{z}$. Under-resolved models (e.g., models with $b_{0}^{z}=10^{13} \mathrm{G}$ simulated at the same resolution) exhibit a slower growth of the MRI. From the growth rates of the MRI, we infer that in 3D the same resolution criterion applies as in the case of axisymmetry.

During early epochs the evolution is similar to that of the corresponding axisymmetric models: a number of radially aligned channels appear. Strong differential rotation causes significant wind-up of flow features leading to structures elongated in $\phi$-direction, i.e., there exists only a modest variation of the MHD variables with azimuthal angle at this stage. Sheet-like structures dominate the field geometry. The rotational profile begins to show distortions due to the transport of angular momentum by Maxwell stresses (see left panel of Fig. 19). At later epochs the flow in $3 \mathrm{D}$ is more complex than in axisymmetry. Although 

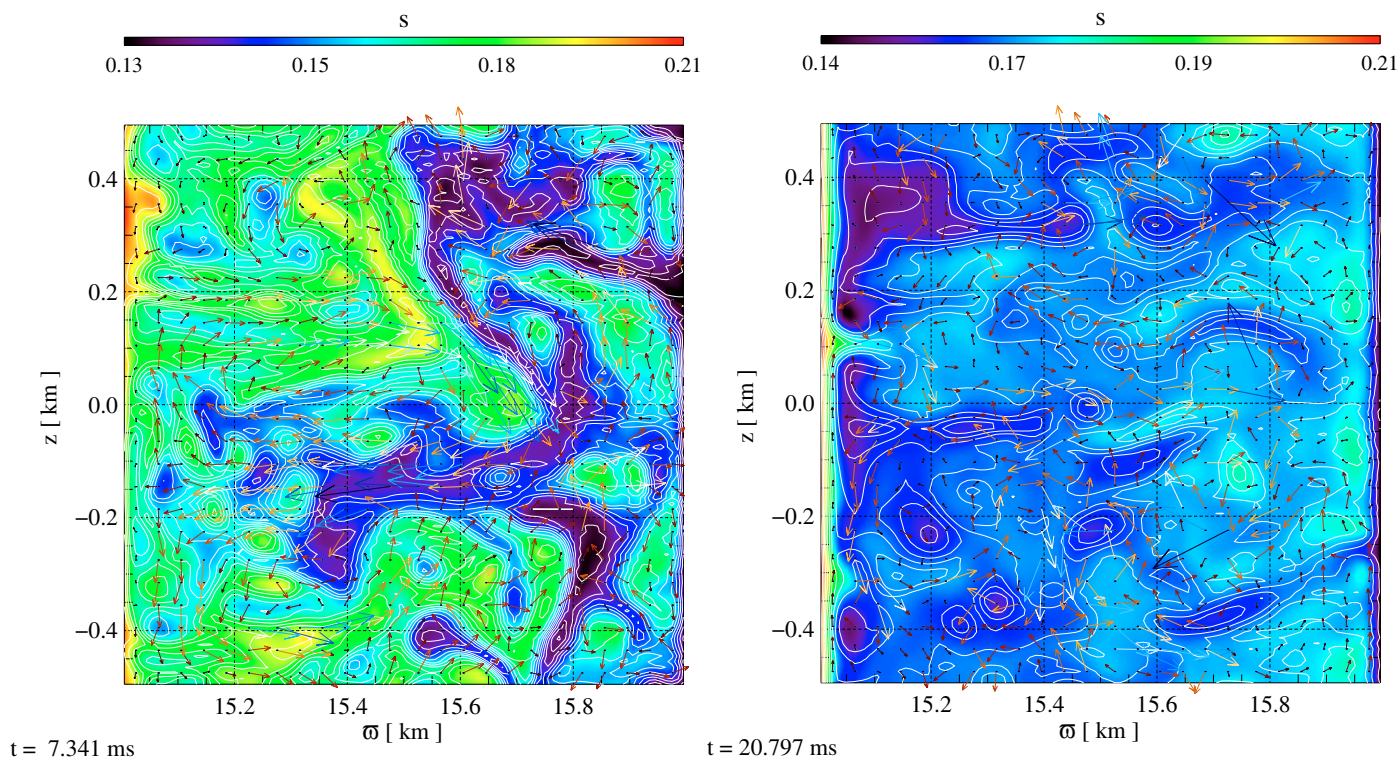

Fig. 18. The entropy distribution (color coded), the poloidal velocity field (arrows), and the magnetic field lines of the model displayed in Fig. 17 at $t=7.3 \mathrm{~ms}$ (left panel) and $t=20.8 \mathrm{~ms}$ (right panel), respectively, i.e., at the begin of the saturation phase and during saturation.

coherent structures, i.e., flux sheets, are still present, their geometry is more tangled and twisted, and less isotropic than earlier in the evolution (see middle panel of Fig. 19).

An evolution from coherent channel flows to a more turbulent state is characteristic for all three-dimensional models with a uniform initial magnetic field. However, as pointed out by Sano \& Inutsuka (2001), channel flows can develop again from the turbulent state. Consequently, the magnetic field can continue growing, and the angular momentum transport will be enhanced strongly. In the most extreme cases, the evolution is similar to that of a corresponding axisymmetric model. This is exactly what we observe for some models at late times, $t \gtrsim 30 \mathrm{~ms}$ (see right panel of Fig. 19), when a dominant channel flow forms. These model enter again a state of exponential growth, and a large part of the angular momentum is extracted by Maxwell stresses. The field strengths reach several $10^{15} \mathrm{G}$, peaking at $10^{16} \mathrm{G}$, and the mean Maxwell stress component $M_{\varpi \phi}$ exceeds $10^{30} \mathrm{erg} \mathrm{cm}^{-3}$ (see middle panel of Fig. 20), and compare with the corresponding axisymmetric model in the left panel). Despite a qualitative similarity between the evolution of the 3D and axisymmetric models, we note that the secondary exponential growth is slower in three dimensions.

The emergence of a large-scale structure of the magnetic field from a turbulent state can be seen in Fig. 21 comparing the field structure at $t=26.8 \mathrm{~ms}$ and $t=42.5 \mathrm{~ms}$, respectively. At the earlier time (left panel), we find a small-scale field dominated by slender flux tubes. Field lines of different polarity (indicated by different colors) are lying close to each other. After the development of the channel flow (right panel), the field is dominated by a large-scale pattern. A smooth surface permeating the box at nearly constant $z$-coordinate separates in two large regions field lines of different polarity from each other. In each of the two regions, we find one broad flux sheet where most of the magnetic energy is concentrated. The separation layer is filled by gas rotating nearly uniformly at a ow angular velocity $\left(\Omega \sim 1500 \mathrm{~s}^{-1}\right)$. The surrounding gas rotates uniformly as well, but at a much higher velocity $\left(\Omega \sim 1800 \mathrm{~s}^{-1}\right)$. The two flux sheets form a thin transition region between both rotational states. Thus, the dynamics is similar to that of the corresponding axisymmetric model.
Because our boundary conditions allow for a loss of angular momentum, and thus for the total disruption of the differential rotation profile by transport through the radial boundaries, this stage represents the end of the evolution, just as it did in axisymmetry: the instability has used up its free-energy reservoir. Hence, the later evolution consists only of violent oscillations.

Only a subset of our models show a prominent re-appearance of single channel flows, and most of them do not exhibit a secondary exponential growth phase. Instead, the mean magnetic energy and the Maxwell stress remain roughly constant during saturation, albeit fluctuating strongly (see the right panel of Fig. 20). Angular momentum transport is less efficient for these models, and their initial rotation profiles remain nearly unchanged. A turbulent flow and magnetic field persist during saturation, and coherent, channel-like structures develop transiently. The structure of the magnetic field of a model with $b_{0}^{z}=4 \times 10^{13} \mathrm{G}$ computed on a grid of $50 \times 100 \times 50$ zones is displayed at two different epochs in Fig. 22. At $t=21.5 \mathrm{~ms}$ (left panel) one recognizes a turbulent state, while large-scale patterns (right panel; yellow structures) dominate the flow at $t=37.2 \mathrm{~ms}$ when the magnetic field strength is largest, and the Maxwell stress is strongest. Unlike in the model discussed above, the coherent flow is unstable and becomes turbulent within a few milliseconds, and the absolute value of the Maxwell stress $\left|M_{\varpi \phi}\right|$ decreases.

Channel modes and parasitic instabilities. The appearance and stability of single large-scale flows that lead to a secondary exponential growth phase and eventually to the disruption of the rotation profile depend on the geometry of the simulated domain as well as on the ratio of the grid resolution and the fastest growing mode.

Models, which are computed in a box of $1 \mathrm{~km}^{3}$ with a resolution of $20 \mathrm{~m}$ and where velocity damping is applied, develop secondary stable channels, if the initial magnetic field is stronger than $2 \times 10^{13} \mathrm{G}$. The MRI growth rates found for these models $\left(\sigma=\{0.76,1.03,1.10\} \mathrm{ms}^{-1}\right.$ for $b_{0}=\{1,2,4\} \times 10^{13} \mathrm{G}$, respectively) indicate that the grid resolution is sufficiently fine to resolve the fastest growing MRI mode for the two most strongly magnetized models. However, it is too coarse for the model with 

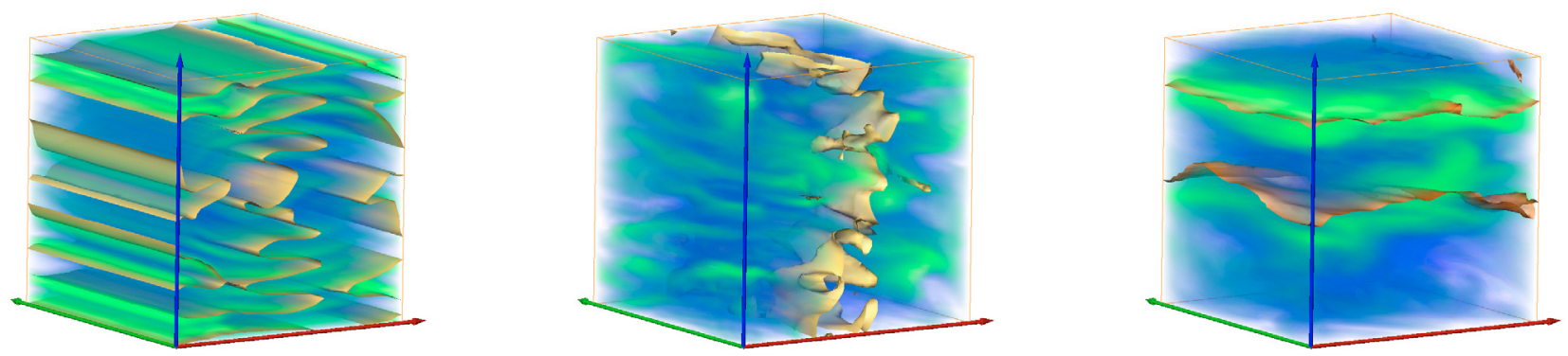

Fig. 19. Structure of a 3D model with $b_{0}^{z}=2 \times 10^{13} \mathrm{G}$ computed on a grid of $1 \mathrm{~km}^{3}$ at a resolution of $20 \mathrm{~m}$ at $t=16.2 \mathrm{~ms}($ left $), t=26.8 \mathrm{~ms}$ middle), and $t=42.5 \mathrm{~ms}$ (right), respectively. Shown is the volume rendered magnetic field strength (blue to green), and a red-orange iso- $\Omega$ surface corresponding to $\Omega=1820 \mathrm{~s}^{-1}$ (left and middle) and $\Omega=1680 \mathrm{~s}^{-1}$ (right), respectively. The red, green, and blue axes point into $\varpi$, $\phi$, and $z$ direction, respectively. Channel flows can be identified as green sheet-like structures.
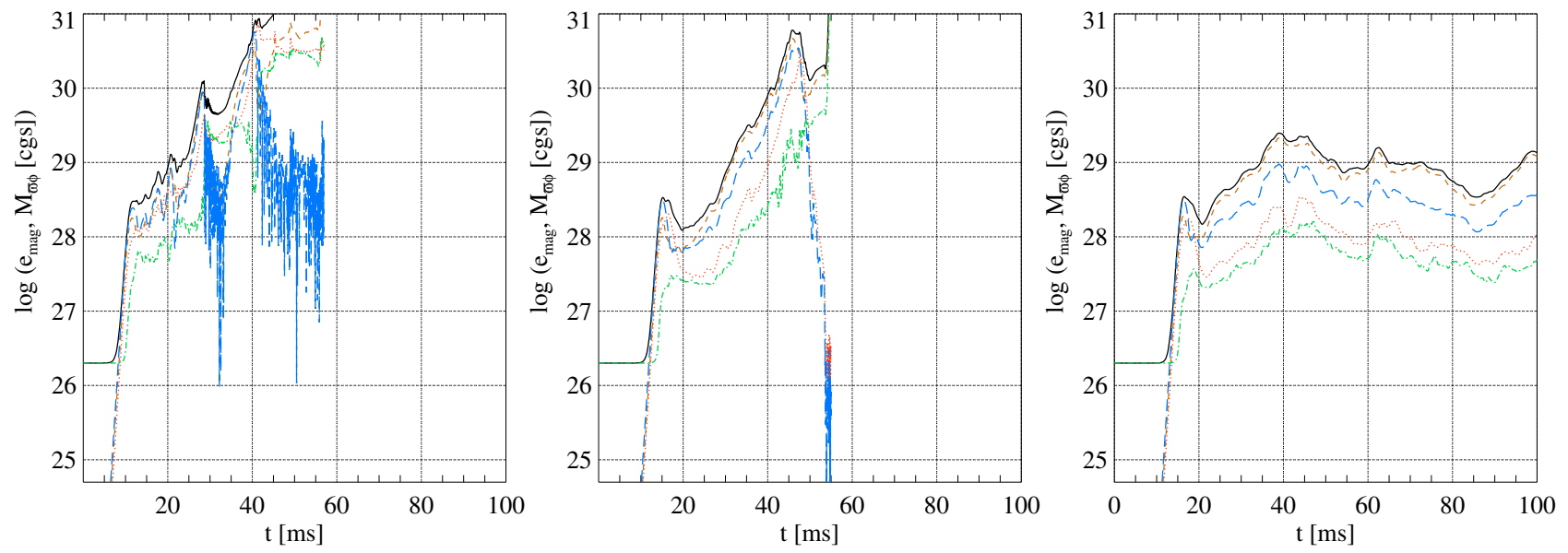

Fig. 20. Evolution of the mean magnetic energy density e $\mathrm{e}^{\text {mag }}$ (solid black line), the mean energy densities corresponding to the $\varpi$ (dotted red), $\phi$ (dashed brown), and $z$ (dash-dotted green) component of the magnetic field, and the absolute value of the mean Maxwell stress component $M_{\varpi, \phi}$ (dashed blue line) for models with an initially uniform magnetic field $b_{0}^{z}=2 \times 10^{13} \mathrm{G}$ in $z$-direction, and a rotation law given by $\Omega_{0}=1900 \mathrm{~s}^{-1}$ and $\alpha_{\Omega}=-1.25$. The panels show a 2D model computed in a box of $L_{\sigma} \times L_{z}=1 \mathrm{~km} \times 1 \mathrm{~km}$ (left), a 3D model computed in a box of $L_{\varpi} \times L_{\phi} \times L_{z}=1 \mathrm{~km} \times 1 \mathrm{~km} \times 1 \mathrm{~km}$ (middle), and a 3D model computed in a box of $L_{\varpi} \times L_{\phi} \times L_{z}=1 \mathrm{~km} \times 2 \mathrm{~km} \times 1 \mathrm{~km}($ right), respectively. The grid resolution is $20 \mathrm{~m}$ in all three cases.
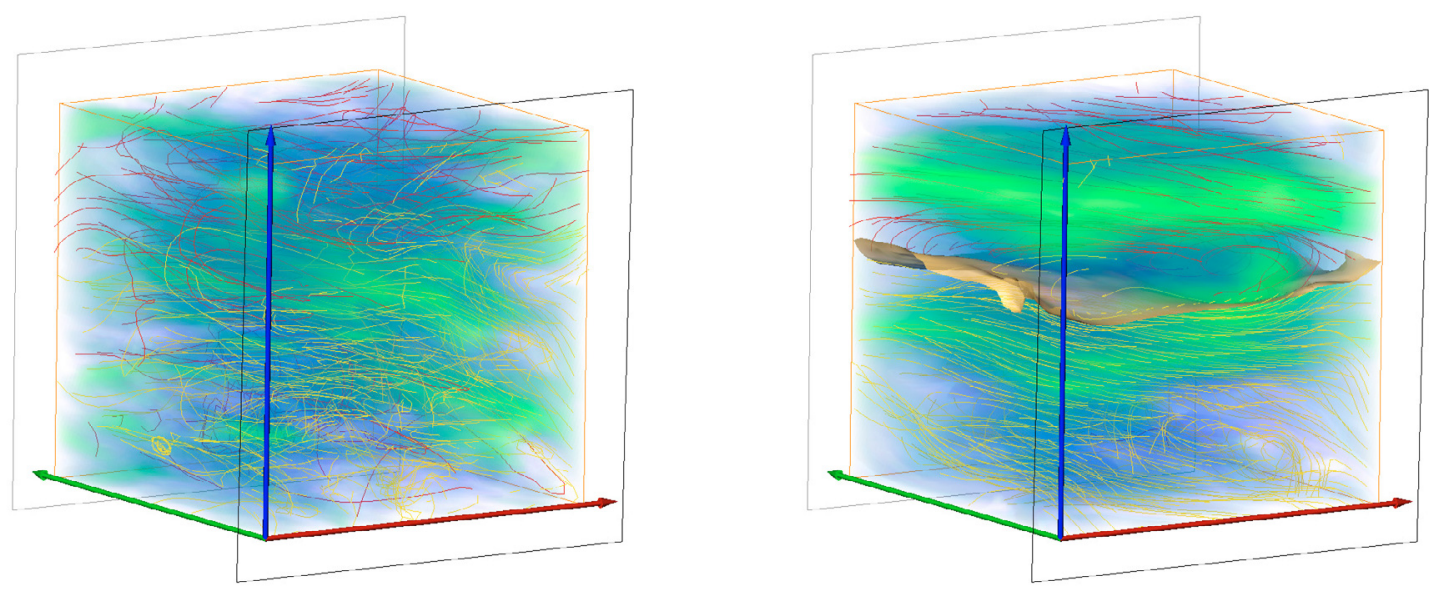

Fig. 21. Same as middle and right panel of Fig. 19, but showing besides the volume rendered magnetic field strength (blue to green) also the magnetic field lines, which are obtained by starting the integration of the magnetic field at two surfaces of constant $\phi$-coordinate (i.e., orthogonal to the green axis) at the left and right hand side of the domain. The field lines originating from the left and right surface are plotted in red and yellow, respectively. The right panel shows, in addition, the isosurface $b_{\phi}=0$ (i.e. the magneto-pause). 

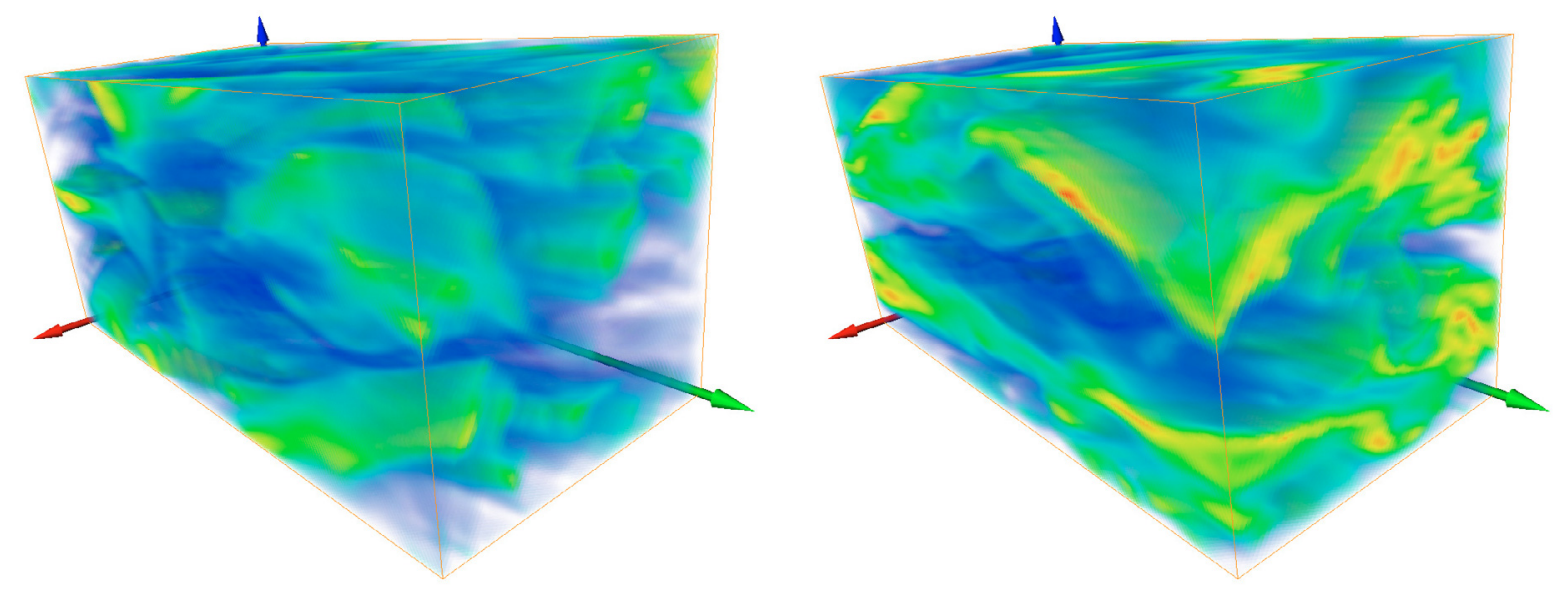

Fig. 22. Volume rendered magnetic field strength of a model with $b_{0}^{z}=4 \times 10^{13} \mathrm{G}$ computed in a box of $1 \times 2 \times 1 \mathrm{~km}^{3}$ with a resolution of $20 \mathrm{~m}$ at $t=21.5 \mathrm{~ms}($ left $)$ and $t=37.2 \mathrm{~ms}($ right $)$, respectively. The coordinate directions are indicated as in Fig. 19.

the weakest initial field, because the theoretical growth rate for the fastest growing MRI mode is $\sigma_{\text {MRI }}=1.14 \mathrm{~ms}^{-1}$ for these models (see Sect. 4.2.1).

To investigate the stability properties of large-scale channel modes as a function of the box geometry, we simulated models with an initially uniform magnetic field using boxes of different size and shape. The models were rotating according to $\Omega_{0}=1900 \mathrm{~s}^{-1}$ and $\alpha_{\Omega}=-1.25$, and their initial magnetic field was $b_{0}^{z}=4 \times 10^{13} \mathrm{G}$ when applying velocity damping boundaries, and $b_{0}^{z}=2 \times 10^{13} \mathrm{G}$ otherwise. We varied both the ratio between the radial and vertical, $L_{\varpi} / L_{z}$, and the radial and toroidal size, $L_{\phi} / L_{\pi}$, size of the box. The grid resolution was $20 \mathrm{~m}$ (see Table B.3). Plotting the stress ratios $M_{\varpi \phi}^{\max } / M_{\varpi \phi}^{\text {term }}$ (Fig. 23; damping boundaries) and $\left\langle M_{\varpi \phi}\right\rangle / M_{\varpi \phi}^{\text {term }}$ (Fig. 24; nondamping boundaries) as a function of the aspect ratio of the computational box, provides some indication of the range of $M_{\varpi \phi}$ values prevailing during the post-growth phase. The ratios allow one to distinguish models with a strong variability due to the dominant re-appearance of channel modes from those models exhibiting a smooth evolution without dominant large-scale coherent structures.

We find that models with a radial aspect ratio $L_{\varpi} / L_{z}=1$ and a toroidal aspect ratio $L_{\phi} / L_{z} \geq 2$ are unstable against parasitic instabilities, independent of the grid resolution in toroidal direction. Turbulence develops and leads to a flow structure as shown in Fig. 22. Models having the same radial aspect ratio, but a smaller toroidal one are stable and evolve similarly as axisymmetric models, i.e., parasitic instabilities do not grow and a dominant large-scale channel flow develops, which gives rise to a morphology of the type presented in Fig. 22. These findings do not depend on how the growth of the MRI ends, i.e., whether velocity damping is applied and reconnection between adjacent channels occurs inside the box, or whether no damping is imposed and reconnection occurs near the surface of the computational box.

These results can be understood from the analysis of parasitic instabilities by Goodman \& Xu (1994), who argued that three-dimensional flows are unstable against parasitic instabilities, but these instabilities can be suppressed by the geometry of the computational box. According to their analysis, the growth rate of the parasitic instabilities is highest for modes with half the wave number of the unstable MRI modes they are feeding

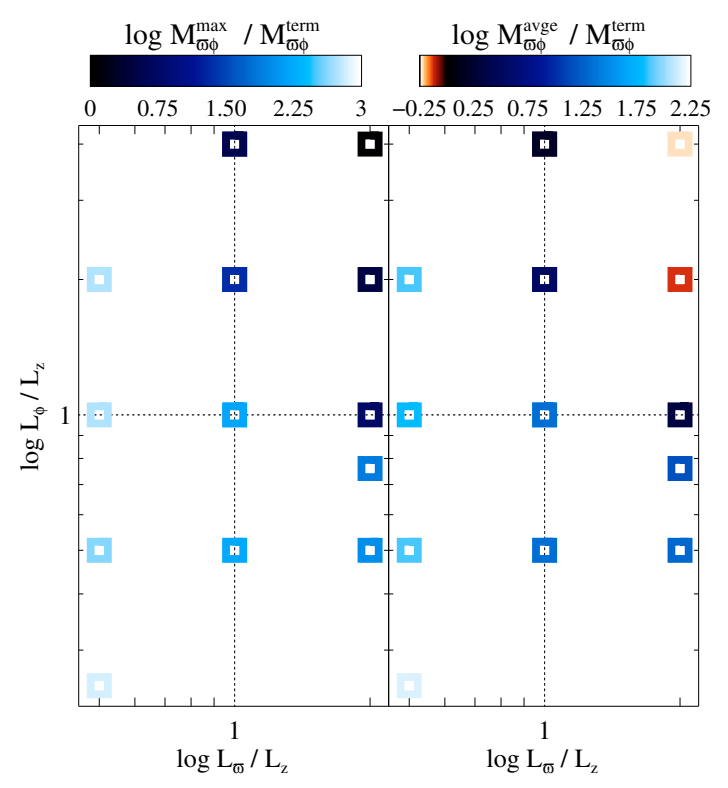

Fig. 23. The left panel shows the ratio of the maximum Maxwell stress per unit volume, $M_{\varpi \phi}^{\max }$, and its value at MRI termination, $M_{\varpi \phi}^{\text {term }}$, as a function of the toroidal and radial aspect ratios, $L_{\phi} / L_{z}$ and $L_{\varpi} / L_{z}$ for the models listed in Table B.3. The right panel shows the ratio of $M_{\varpi \phi}$ averaged over the saturation phase and the value at MRI termination. Each model is represented by a symbol its color reflecting its maximum Maxwell stress. All models are computed imposing velocity damping at the radial grid boundaries.

off. Hence, if a channel flow forms at late times with a wavelength equal to the box size in $z$-direction, $L_{z}$, unstable parasitic modes must have a toroidal wavelength $\sim 2 L_{z}$ to grow rapidly. Thus results in the criterion for the channel flow instability we have found in our simulations.

In accordance with simulations presented recently by Bodo et al. (2008), we find that models with a radial aspect ratio $L_{\varpi} / L_{z} \leq 1$ experience a second exponential growth phase as described in Sect. 4.2 (note the large ratios of $M_{\varpi \phi}^{\max }$ and $M_{\varpi \phi}^{\text {term }}$ for the corresponding models in Fig. 23), whereas a larger radial aspect ratio appears to favor a less violent post-growth phase where coherent channel modes can appear but are disrupted after a short time. Bodo et al. (2008) obtained this result for simulations performed with a toroidal aspect ratio $L_{\phi} / L_{z}=4$. 


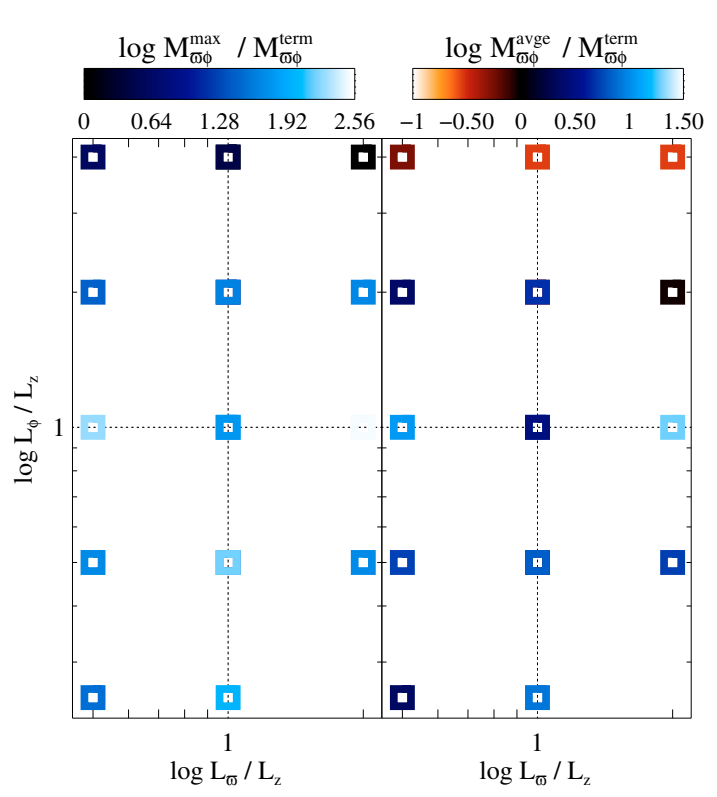

Fig. 24. Same as Fig. 23, but for models where no velocity damping is applied at the radial grid boundaries.

We confirm a similar dependence of the dynamics on the radial aspect ratio also in axisymmetry and for three-dimensional boxes with smaller $L_{\phi} / L_{z}$. In this case, parasitic instabilities are unable to disrupt the channel modes. Consequently, the MRI experiences a second exponential growth phase dominated by just one (two in a few cases) large channel mode of width $a$ which is determined by the size of the computational box in $z$-direction. The maximum Maxwell stress that can be reached is limited by the onset of resistive instabilities. The dependence on the channel width (Eq. (A.11)) explains why the maximum Maxwell stress varies with $L_{z}$ : larger boxes allow for wider channels for which the resistive instabilities grow slower, thus requiring higher Alfvén velocities for a growth rate comparable to the one of the MRI. Hence, the MRI reaches stronger fields for larger (in $z$ direction) boxes.

Despite the differences in the MRI termination process, the behavior of models with and without velocity damping is quite similar, because the velocity damping does not affect the second generation of vigorous channel flows significantly. Thus, the breakup of these channels and the values of the corresponding maximum Maxwell stress do not depend strongly on the choice of the boundary condition. On the other hand, MRI termination (the termination of the initial exponential growth of the MRI) does depend on whether velocity damping is applied or not, and thus the ratio $M_{\varpi \phi}^{\max } / M_{\varpi \phi}^{\text {term }}$, too.

For boxes having a large toroidal aspect ratio, $L_{\phi} / L_{z} \geq 2$, we observe a quiet evolution during the non-linear saturation phase of the MRI when varying the radial aspect ratio, $L_{\varpi} / L_{z}$. In particular, the fluctuations of $M_{\varpi \phi}$ are small after MRI termination for models where both aspect ratios are large. The models in the upper right corner of Fig. 23 have values of $\left\langle M_{\varpi \phi}\right\rangle / M_{\varpi \phi}^{\text {term }}$ close to unity.

We may try to infer some consequences from these results for the MRI in supernova cores. Close to the core's equator the region, where the MRI develops, can have to a small radial size, $L_{\varpi}$, ranging from a few to a few hundred kilometers determined by the gradients of $\Omega$ and $S$ in the core. The vertical extent of the unstable region, $L_{z}$, can be expected to be of similar size. The azimuthal extent of the MRI unstable region, $L_{\phi}$, will be significantly larger, leading to a non-violent evolution of the saturated state of the MRI. The geometry is different close to the pole. However, we cannot apply our results there without modifications as we have considered only cases where the gradients of $\Omega$ and of all thermodynamic quantities are aligned - a situation which does not apply near the poles.

Effects of resolution and initial magnetic fields in 3D vs 2D. After having discussed how the aspect ratios of the simulation box determine the Maxwell stress at MRI termination and during the subsequent saturation phase, we will now compare whether the behavior of 3D models differs from that of 2D models. The models discussed in this paragraph are listed in Tables B.3 and B.4.

First, we note that in 3D, as in axisymmetry, the growth rate of the instability is not affected by the choice of the grid provided the fastest growing mode is resolved.

Figure 25 demonstrates that in 3D the dependence of $M_{\varpi \phi}^{\text {term }}$ on the initial magnetic field strength is well described by the same power law as in axisymmetry: models without damping of the radial velocity line up along a band $\propto\left(b_{0}^{z}\right)^{16 / 7}$, and models with velocity damping are characterized by a roughly constant value of $M_{\varpi \phi}^{\text {term }}$, which depends on the size of the radial box (compare with Fig. 8). This agreement is to be expected as the growth and the resistive disruption of channel flows are essentially axisymmetric processes, which are, thus, not significantly modified by three-dimensional effects.

After MRI termination the evolution of $M_{\varpi \phi}$ depends on the aspect ratio of the computational box (see discussion above). When averaging the fluctuating Maxwell stresses over the saturation phase, we find values for $\left\langle M_{\varpi \phi}\right\rangle$ which differ considerably from those of $M_{\varpi \phi}^{\text {term }}$. Lacking a thorough understanding of the instabilities involved in the MRI saturation process, and having only a imited set of 3D models at hand, one is not yet in a position to formulate a better description of the dependence of the evolution after MRI termination on the aspect ratio of the box, and to provide a unified description of MRI saturation amplitudes.

\subsubsection{Uniform $b^{z}$ field, entropy gradients}

Mixed regime: let us first consider models from the mixed regime having an initial rotation law given by $\Omega_{0}=1900 \mathrm{~s}^{-1}$ and $\alpha_{\Omega}=-1.25$, and an entropy distribution given by $S_{0}=0.2$ and $\partial_{\varpi} S=-0.038$ (i.e, with a negative entropy gradient). In axisymmetry the MRI grows in these models with a rate of $\sigma_{\text {MRI }} \approx 1.7 \mathrm{~ms}^{-1}$, i.e., close to the theoretical value of $\approx 2.0 \mathrm{~ms}^{-1}$. The $3 \mathrm{D}$ models show the same growth rate provided the spatial grid resolution is sufficiently high.

The long-term evolution (i.e., many rotational periods into the non-linear phase) of the models depends strongly on the choice of the radial boundary conditions. If the entropy at the inner and outer boundary is allowed to change (i.e., using reflective boundaries), a flat entropy profile develops after a short time. To reduce the influence of boundary effects, one could employ a technique widely used in simulations of convective layers: add a cooling layer on top of and an overshoot layer below the convection zone. However, exploring this approach was beyond the scope of the present work.

For models having a negative entropy gradient the growth of the MRI is not influenced by 3D effects, if the fastest growing mode is resolved. Thus, their behavior is similar to that of models with no entropy gradient. The Maxwell stress at MRI termination also does not differ significantly from that of the 


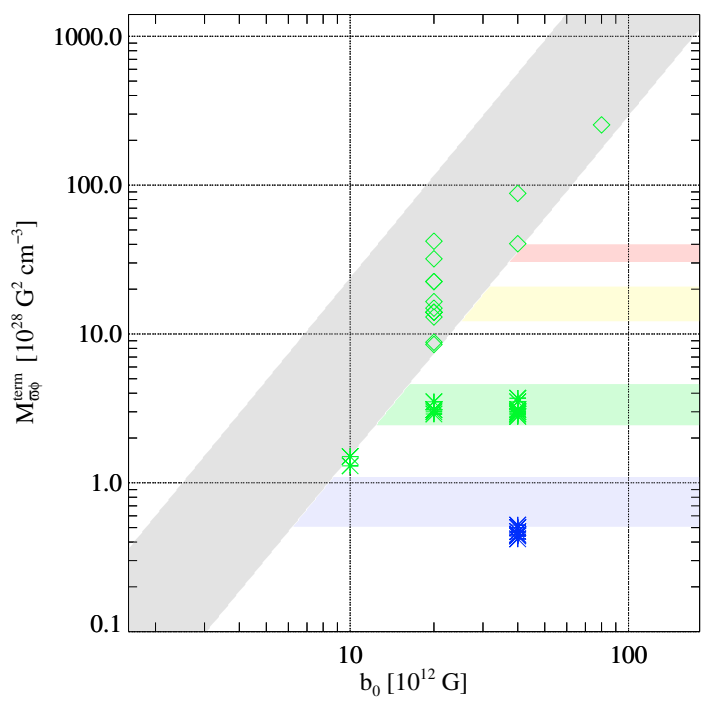

Fig. 25. Volume-averaged Maxwell stress component $M_{\varpi \phi}^{\text {term }}$ at MRI termination as a function of the initial magnetic field strength, $b_{0}$, for axisymmetric models with uniform initial magnetic field in $z$-direction, a rotational profile $\Omega=1900 \mathrm{~s}^{-1} \varpi^{-1.25}$, and a vanishing entropy gradient for a set of 3D models computed with (asterisks) and without (diamonds) velocity damping at the radial boundaries. Models computed in a box with a radial size of $1 \mathrm{~km}$ and $0.5 \mathrm{~km}$ are shown with green and blue symbols, respectively. The colored bands are the same as in Fig. 8.

corresponding axisymmetric models, and due to the boundary conditions applied in the models (velocity damping) its value is set by recombination of field lines close to the inner and outer radial boundary.

Contrary to axisymmetric models, the saturated state of the $3 \mathrm{D}$ models does not show any sign of a late exponential growth phase characterized by the re-appearance of channel modes, and the saturated MRI stresses are smaller in magnitude than $M_{\varpi \phi}^{\text {term }}$, i.e., the maximum Maxwell stress is reached at MRI termination (see Fig. 26 and compare with Fig. 4). The evolution of the average radial entropy profile profile, computed as the average of $S(\varpi, \phi, z)$ at constant $\varpi$, is shown in Fig. 27. Until the saturation of the instability (at $t \approx 11 \mathrm{~ms}$ ), the initial linear profile $S(\varpi)$ is basically unchanged. However, afterward the entropy profile flattens, $S$ becoming nearly constant for $15.2 \mathrm{~km} \leq \varpi \leq$ $15.8 \mathrm{~km}$. Close to both radial boundaries, the entropy profile develops extrema, which are most likely an artifact of our boundary conditions. The flat entropy profile is stable and does not vary strongly with time. The $\Omega$ profile flattens after the initial growth phase, too. The velocity field in the saturated state is dominated by a rich small-scale structure, while the magnetic field is organized in a multitude of flux tubes.

$\mathrm{MBI}$ regime: next we consider a few models that, in axisymmetry, belong to the MBI regime. Initially, the models rotate rigidly with $\Omega=1900 \mathrm{~s}^{-1}$ and possess an entropy gradient $\partial_{\bar{\tau}} S=-0.10 \mathrm{~km}^{-1}$ (i.e., $C=-3.6$ ). We computed models for $b_{0}^{z}=10 \mathrm{G}, 10^{10} \mathrm{G}$, and $2 \times 10^{13} \mathrm{G}$, respectively. All models are simulated in a box of size $L_{\varpi} \times L_{\phi} \times L_{z}=1 \times 2 \times \times 1 \mathrm{~km}^{-3}$ and on a a grid of $50 \times 100 \times 50$ zones.

Contrary to their axisymmetric counterparts (see Sect.4.2), these models develop convective modes even when no magnetic field is present. As described, e.g., by Tassoul (1978), rotation can stabilize axisymmetric modes in a convectively stable environment, but non-axisymmetric modes can nevertheless

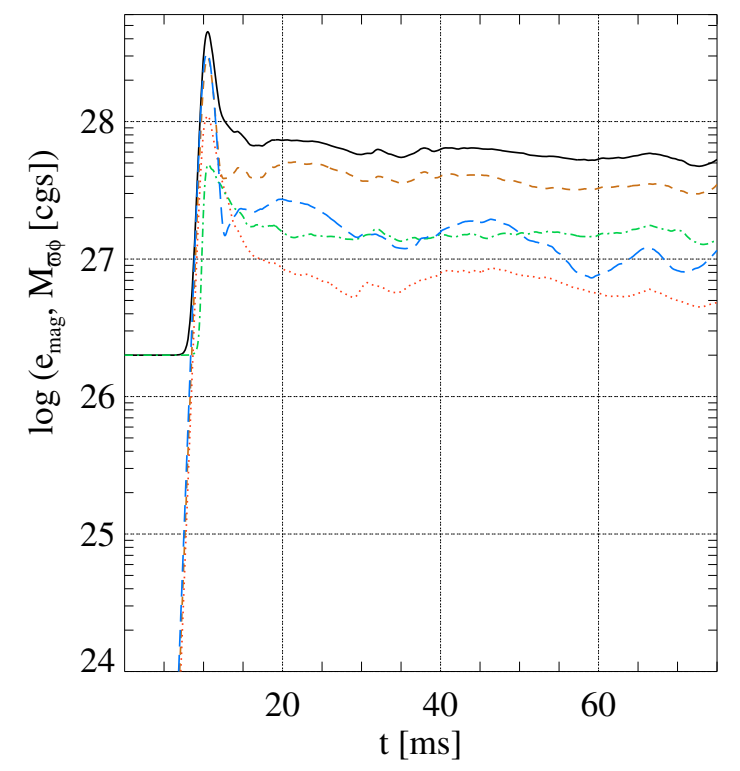

Fig. 26. Evolution of the mean magnetic energy density $\mathrm{e}^{\mathrm{mag}}$ (solid black line), the mean energy densities corresponding to the $\varpi$ (dotted red), $\phi$ (dashed brown), and $z$ (dash-dotted green) component of the magnetic field, and the absolute value of the mean Maxwell stress component $M_{\varpi, \phi}$ (dashed blue line) for a 3D model belonging to the mixed regime. The model rotates differentially with $\Omega_{0}=1900 \mathrm{~s}^{-1}$ and $\alpha_{\Omega}=-1.25$. The initial entropy gradient is $\partial_{\varpi} S=-0.038 \mathrm{~km}^{-1}$, and the initial magnetic field strength is $b_{0}^{z}=2 \times 10^{13} \mathrm{G}$. The model was simulated in a box of size $L_{\varpi} \times L_{\phi} \times L_{z}=1 \times 2 \times 1 \mathrm{~km}^{-3}$ and on a grid of $50 \times 100 \times 50$ zones.

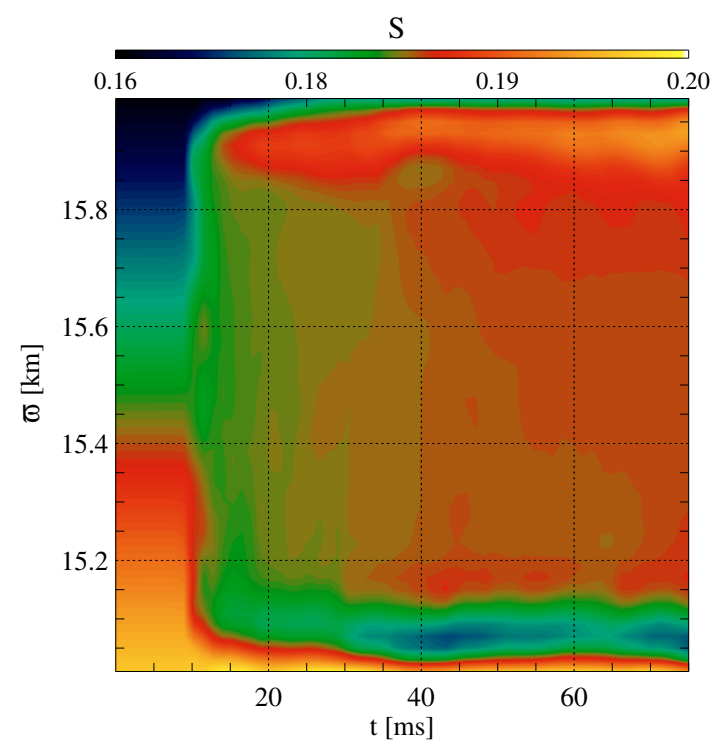

Fig. 27. Average radial entropy profile as a function of time for the model shown in Fig. 26.

grow in that situation. The model with the weakest initial field $\left(b_{0}^{z}=10 \mathrm{G}\right)$ shows a growth of non-axisymmetric MBI modes, but these modes cannot be resolved due to the extremely weak initial field. Thus, the model behaves essentially similar to an unmagnetized one, but can serve as a reference model for initially more strongly magnetized models where we can resolve $\lambda_{\text {MRI }}$. The growing convective modes eventually extend over the entire domain in radial and $z$-direction, while having a small wavelength in $\phi$ direction (see Fig. 28, upper panel). The exponential growth of the convective instability saturates at $t \approx 7 \mathrm{~ms}$. 

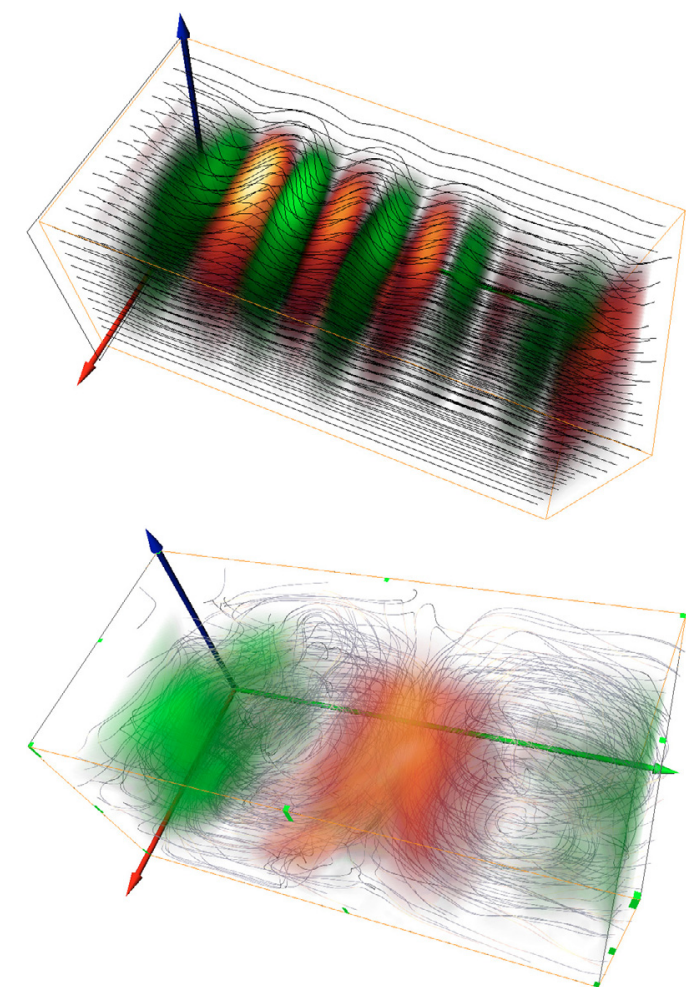

Fig. 28. Flow structure of a MBI model with $b_{0}^{z}=10 \mathrm{G}$ at $t=4.86 \mathrm{~ms}$ (upper panel), and $t=14.4 \mathrm{~ms}$ (lower panel), respectively. The solid black lines are stream lines of the velocity field computed in a frame co-rotating with the mean angular velocity, and regions of positive and negative radial velocity are colored in red and green, respectively. The colored arrows point into the same coordinate directions as in Fig. 19.

During this growth phase the mean magnetic energy increases at the same rate as does the kinetic energy. After MRI termination the structure of the model is characterized by two large, roughly cubic convective cells with a size of about $1 \mathrm{~km}^{3}$ instead of a multitude of elongated structures (see (Fig. 28, lower panel), and an essentially flat entropy profile. The magnetic field is subject to kinematic amplification at a smaller growth rate than before MRI termination due to stretching in the convective vortices. At much later epochs the typical size of structures in the velocity field decreases again, leading to more turbulent fields. The magnetic field, which is too weak to affect the dynamics, is passively advected with the flow.

For the model having the strongest initial magnetic field $b_{0}^{z}=2 \times 10^{13} \mathrm{G}$ we can resolve $\lambda_{\mathrm{MRI}}$. The axisymmetric version of this model showed a MBI growth a rate close to the theoretical one $\left(\sigma_{\text {MRI }} \sim 1.7 \mathrm{~ms}^{-1}\right)$. For the $3 \mathrm{D}$ model we find $\sigma_{\text {conv }} \sim 2.6 \mathrm{~ms}^{-1}$ ), i.e., its evolution is dominated by convection (although we are able to resolve the MBI), and the MBI growth rate is similar to that of a weakly magnetized model (see previous paragraph). MBI growth is mediated by non-axisymmetric modes having the same elongated geometry as those in the essentially unmagnetized model. After saturation, a few large vortices of approximately cubic shape form, which later decay into small-scale structures again. An intermediate stage of this decay process is displayed in Fig. 29, when one large vortex is still present in the right half of the box, while its left half is dominated by spatially less coherent fields. At even later times the vortex disappears and the structure of the whole model is similar to that shown in the left half of the box. The mean magnetic energy and Maxwell stress are small compared to typical MSI or mixed models. Compared to a differentially rotating model

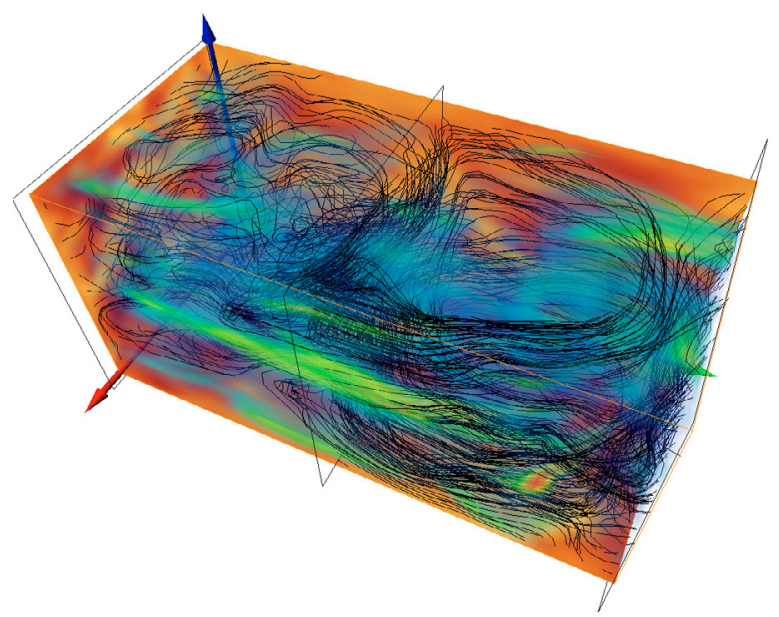

Fig. 29. Structure of a rigidly rotating model with $b_{0}^{z}=2 \times 10^{13} \mathrm{G}$ at $t=19.3 \mathrm{~ms}$. The figure shows a volume rendering of the magnetic field strength (blue - green), the value of the angular velocity, $\Omega$, at three slices parallel to the coordinate axes (red - yellow), and stream lines of the velocity field in a co-rotating frame (black lines). In the right half of the figure, one can identify one large convective cell, whereas the features of, e.g., the velocity field in the left half are of considerably smaller scale. The magnetic field is comparably weak inside the convective cell, and strongest in the flux tubes both at the boundaries of the convective cell and in the left part. The colored arrows point into the same coordinate directions as in Fig. 19.

$\left(\Omega_{0}=1900 \mathrm{~s}^{-1}, \alpha_{\Omega}=-1.25\right)$ with a vanishing entropy gradient, the maximum magnetic fields are reduced by a factor of $\lesssim 2$, and the mean magnetic energies and Maxwell stresses by a factor of $\sim 10$, but we still find a slow growth at the end of the simulation.

Finally, we add a few comments on a model where we cannot resolve $\lambda_{\text {MRI }}\left(b_{0}^{z}=10^{10} \mathrm{G}\right)$, but where the magnetic field saturates within $\sim 60 \mathrm{~ms}$ after the onset of convection. The model evolves similarly to the essentially unmagnetized one, but at $t \approx 60 \mathrm{~ms}$ the energy of the kinematically amplified magnetic field becomes almost as high as the convective kinetic energy. The amplification process ceases, and the magnetic energy levels off. Close to the end of the simulation convective transport gives rise to a rotation law $\Omega \approx$ const..

\subsubsection{Magnetic fields with zero net flux}

Many results discussed above also apply analogously to models where the initial magnetic field has a vanishing net flux through the surface of the computational box. We simulated models with $\Omega=1900 \mathrm{~s}^{-1}$ and $\alpha_{\Omega}=-1.25$, and find a basically axisymmetric growth of channel flows, which decay due to resistive instabilities at a level consistent with axisymmetric models. The late evolution of the models is dominated by turbulent fields. Contrary to uniform-field models, there is no second phase of channel activity. Hence, the mean magnetic energies and Maxwell stresses do not fluctuate violently in the saturated state, and - even when no velocity damping is applied - the maximum values of, e.g., $\left|M_{\varpi \phi}\right|$, are reached at MRI termination. Later on $\left|M_{\varpi \phi}\right|$ decreases by a factor of a few, and stays roughly constant subsequently.

Performing similar simulations Lesur \& Ogilvie (2008) proposed a non-linear dynamo that balances the dissipation of the magnetic energy in MRI models with zero net flux. In the turbulent saturated state, they identified large-scale spatially (over a sizable fraction of the box) and temporally (over several rotational periods) coherent patterns of the toroidal magnetic field. 


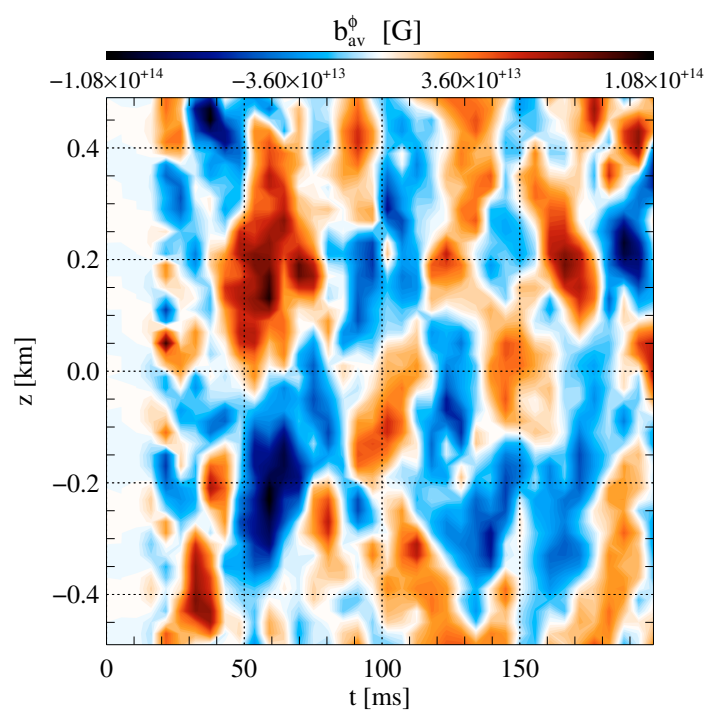

Fig. 30. Evolution of large-scale coherent patterns exhibited by the mean (i.e., averaged over a plane at constant $z$-coordinate) toroidal field component of a 3D model having an initial magnetic field of zero flux and strength $b_{0}^{z}=2 \times 10^{13} \mathrm{G}$. The model was simulated in a box of $1 \mathrm{~km}^{3}$ covered by $50^{3}$ zones with no velocity damping applied. This figure is similar to Fig. 1 of Lesur \& Ogilvie (2008).

To study this process, we looked for similar patterns in our models.

An example is shown in Fig. 30 for a model with $b_{0}^{z}=$ $2 \times 10^{13} \mathrm{G}$ simulated in a box of $1 \mathrm{~km}^{3}$ on a grid of $50^{3}$ zones applying no velocity damping. The figure shows the mean value of the toroidal field component $b_{\mathrm{av}}^{\phi}$ (i.e. $b^{\phi}$ averaged over $\varpi$ and $\phi$ ) as a function of $z$ and time. For $t \lesssim 14 \mathrm{~ms}$ the early channels flows can be identified in which the magnetic field grows. At $t \approx 14 \mathrm{~ms}$, the channels are disrupted, and the growth of the field seizes. In the saturated state that follows, the mean (vertical) size of the structures is larger: at any time, we find only a few (typically two) regions of opposite field polarity (blue and red), which remain stable for a few rotational periods $\left(\Omega^{-1} \approx 0.53 \mathrm{~ms}\right)$. Thus, we make similar observations as Lesur \& Ogilvie (2008) do for their models.

We also simulated a few 3D models with zero-flux fields in the mixed regime. The results are analogous to those obtained if the initial fields are uniform(see, e.g., Fig. 31). The MRI growth rates are similar to those of the corresponding 2D models, and the mean Maxwell stress in the saturated state is somewhat smaller. In the saturated state, both the entropy (upper panel) and the angular velocity profiles become flat rapidly. The spatially and temporally coherent large-scale structures in the magnetic field are even more pronounced than in MSI models (compare the lower panel of Fig. 31 with Fig. 30). They consist of two large regions characterized by an opposite sign of $b^{\phi}$, which persist for the entire simulation of the saturated state $(\approx 140 \mathrm{~ms})$, subject only to a slow drift in vertical direction. The implications of this behavior for the presence and properties of a nonlinear dynamo of the type proposed by Lesur \& Ogilvie (2008) remain to be explored.

\section{Summary and conclusions}

We have studied the possible amplification of seed perturbations in supernova cores by the magneto-rotational instability. If the MRI grows on dynamically relevant time scales (a few tens of

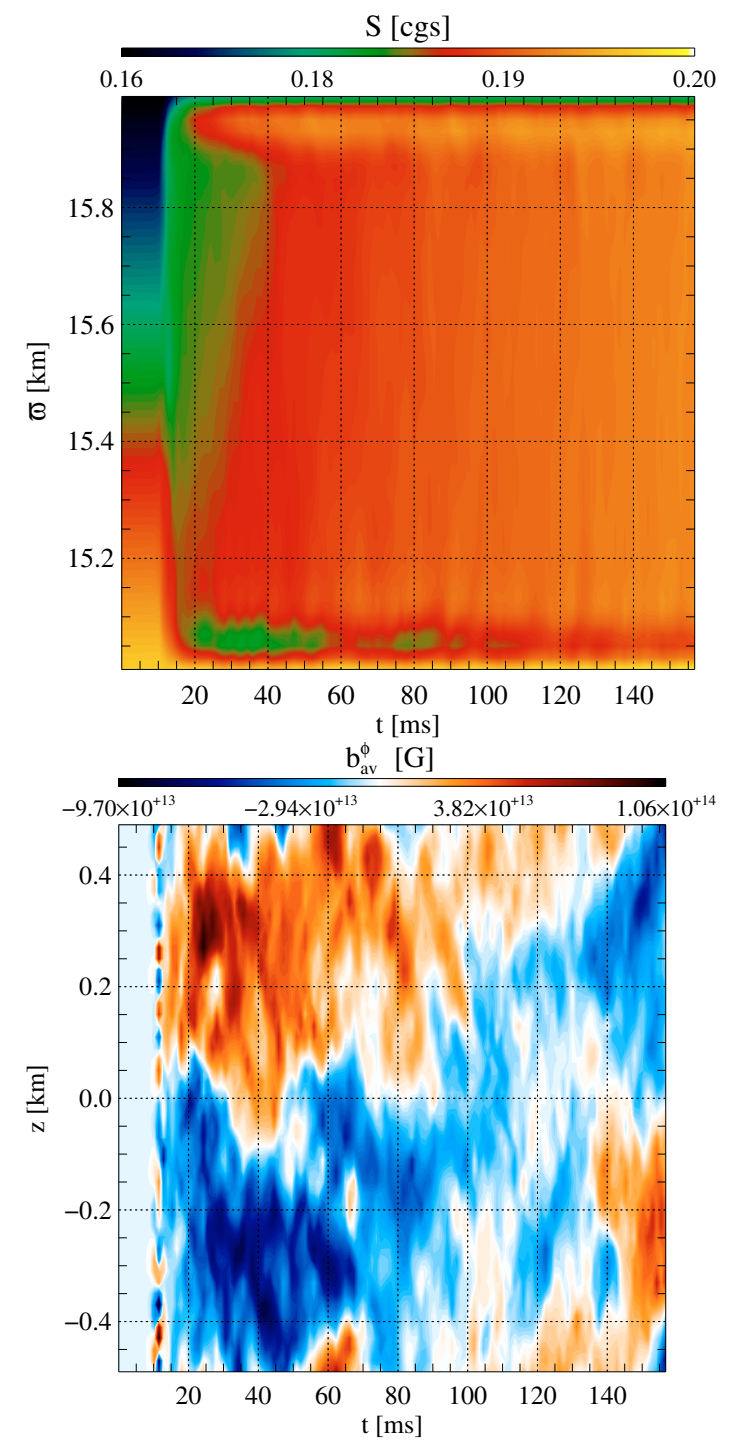

Fig. 31. Evolution of a mixed-regime 3D model with zero net flux $\left(b_{0}^{z}=\right.$ $2 \times 10^{13} \mathrm{G}$ ), an entropy gradient $\partial_{\varpi} S=-0.038 \mathrm{~km}^{-1}$, and a rotation law given by $\Omega=1900 \mathrm{~s}^{-1}$ and $\alpha_{\Omega}=-1.25$. The upper panel shows the entropy of the model averaged over planes of constant $\varpi$ as a function of radius and time. The lower panel shows, similarly to Fig. 30, the average of $b^{\phi}$ over planes of constant $z$ as a function of $z$ and time.

milliseconds), it can lead to MHD turbulence and efficient transport of angular momentum. Because the growth of the magnetic field and the associated Maxwell stresses is exponential in time, the MRI is one of the most promising mechanisms to amplify the - most likely weak - magnetic field of the supernova progenitor up to dynamically relevant strengths.

As pointed out by Akiyama et al. (2003), the conditions for the instability are fulfilled in typical post-collapse supernova cores. Under the assumption that the MRI converts most of the energy contained in differential rotation into magnetic energy, these authors predicted saturation fields of approximately $10^{15} \mathrm{G}$. This prediction derived from a semi-analytic analysis and 1D simulations can only be confirmed by detailed multidimensional numerical simulations. The reliability of global simulations of the entire core, however, is limited due to the necessity to resolve accurately small length scales (a few meters, at most) leading to impracticable computational costs. 
Traditionally, the MRI is studied in great detail in accretion discs, i.e., in systems dominated by Keplerian rotation. Because typical post-collapse supernova cores differ from these systems in many respects, e.g., by the importance of the thermal stratification and the sub-Keplerian rotation, we investigated the instability under more general physical conditions. Analyzing the MRI dispersion relation of Balbus (1995); Urpin (1996), we identified the regimes of the instability relevant to supernova cores.

We distinguish between Alfvén and buoyant modes of the MRI. The former ones are generalizations of the standard MRI modes, and the latter ones resemble standard convective modes. Buoyant modes are unstable only in systems dominated by a negative entropy gradient, whereas Alfvén modes prevail if differential rotation is the main agent of the instability. Whereas Alfvén modes are rapidly amplified only for a small range of wave numbers, buoyant modes grow at essentially the same rate for a wide range of wave numbers, $k \leq k_{\max }$.

We have identified six regimes of the MRI depending on the ratio of the entropy and angular velocity gradient. These MRI regimes and their properties can be summarized as follows:

1. Sufficiently large positive gradients of the angular velocity or of the entropy define the stable regime with oscillatory rather than growing modes.

2. For sufficiently strong differential rotation and small entropy gradients (or small buoyancy frequencies), we find the shear regime, corresponding to the hydrodynamic shear instability.

3. If negative entropy gradients dominate the system, it is located in the convective regime, which resembles ordinary hydrodynamic convection potentially modified in the nonlinear phase by the presence of a magnetic field.

4. A small degree of differential rotation (e.g., Keplerian) and a small entropy gradient (if present at all) are the conditions for the magneto-shear (MSI) regime, well studied for accretion discs.

5. When fast (nearly) rigid rotation suppresses convection, its stabilizing effect can be overridden by a weak magnetic field, giving rise to magneto-buoyant (MBI) modes. This regime is only encountered in axisymmetric flows as rotation can stabilize only axisymmetric modes of convection, i.e., in three dimensions convection may grow faster than the MBI.

6. Finally, a mixed regime exists which shares properties of all unstable regimes listed above.

To substantiate our stability analysis, we performed a set of more than 200 models of semi-global high-resolution simulations of the MRI in simplified models of post-bounce cores. Our novel semi-global simulations combine elements of both global and local simulations by taking into account the presence of global background gradients and by providing high local spatial resolution. In particular, we employed the shearing-disc boundary conditions proposed by Klahr \& Bodenheimer (2003), which allow for the treatment of global gradients of, e.g., density or entropy, and studied the influence of a thermal stratification on the MRI assuming various (radial) entropy profiles. The presence of gradients constitutes an important difference of our setting from that of accretion discs. We used a newly developed Eulerian highresolution MHD code to evolve the flow in a computational box having an edge length of a few kilometers. The box was located in the equatorial plane of the core at a distance of $15 \mathrm{~km}$. The initial data were computed assuming hydrostatic equilibrium of differentially rotating matter described by a simplified equation of state. The gas in the box was endowed with a weak initial magnetic field of different topology and strength. In most of the simulations, the magnetic field of the progenitor had a strength of approximately $10^{10} \mathrm{G}$. We neglected the effects of neutrino radiation and assumed an ideal MHD flow.

Computed under the assumption of rotational equilibrium, our background models differ from realistic supernova cores in many respects. One of the most important shortcomings is the neglect of the large-scale accretion flow through the postshock region onto the proto-neutron star. From a physical point of view, such flows, whose influence on the MRI has not been studied previously, add to the already considerable complexity of the problem considered here, possibly allowing for additional regimes and modifying growth and saturation of the instability. Technically, the proper treatment of the boundary conditions required for a correct modeling of such flows in non-global simulations is rather involved. Thus, we have decided to postpone the study of the MRI in the presence of a large-scale accretion flow and focus on systems without overall radial motions.

The main results of our simulations are agree well with both our mode analysis and with local simulations of the MRI in accretion discs. They also confirm the estimates of Akiyama et al. (2003), and they are consistent with the results of global MHD simulations of core collapse (e.g., Obergaulinger et al. 2006b,a). We summarize our results as follows:

1. Under the physical conditions considered in this study, i.e., in a background rotating in hydrostatic equilibrium, the MRI can act in supernova cores amplifying an initial magnetic field strongly. The growth times are approximately equal to the rotational period of the core, which for rapidly rotating cores is sufficiently fast to influence the dynamics.

2. Due to our relatively fine numerical grids, we were able to resolve the fastest growing MRI modes for initial field strengths higher than a few $10^{12} \mathrm{G}$. This threshold is considerably lower than the one of previous global simulations (e.g., Obergaulinger et al. 2006b,a), enabling us to probe the MRI in a parameter range inaccessible to global simulations.

3. The growth of the instability is accompanied by the development of channel flows, predominantly radial flows of alternating direction stacked up in the $z$-direction. This flow pattern is characteristic of both axisymmetric and threedimensional simulations. The width of the channels is set by the wave length of the fastest growing MRI modes.

4. At MRI termination (i.e., at the end of the first exponential growth phase of the instability) the channels dissolve into a turbulent flow having a complex magnetic field topology. During the subsequent evolution secondary generations of channel flows can (re-) appear which characterize secondary phases of exponential growth.

5. We identified the mechanism responsible for the breakup of the channel flows and MRI termination in our simulations. Despite the absence of a physical resistivity in our model equations, we find that resistive MRI instabilities of the tearing-mode type develop due to the finite numerical resistivity of our MHD code. A main characteristic of the channel flows is the presence of prominent current sheets immersed between layers of opposite magnetic polarity, which are unstable against current-driven instabilities. Using a simplified model of this kind of flows, we investigated the growth rates, $\sigma_{\mathrm{r}}$, of the resistive instabilities, and derived an approximate law for the scaling of $\sigma_{\mathrm{r}}$ with the magnetic field strength present in the channels, the channel width, and the grid resolution. Comparing these growth rates with the MRI ones, we find that the MRI ceases to grow once the tearing modes grow faster than the MRI. Using this criterion, we are able to 
explain the dependence of the conditions (i.e., field strength, Maxwell stresses) at MRI termination on, e.g., the initial field strength, the grid resolution, and the initial rotation profile.

Strictly speaking, there should be no reconnection without physical resistivity, and the behavior of a magnetized ideal fluid subject to numerical resistivity may be quite different from that of a fluid having a large but finite conductivity. Consequently, our results and their implications cannot replace a rigorous treatment of MRI growth in supernova cores with a non-ideal MHD model. In particular, we have to be careful when drawing conclusions for the MRI in nonideal plasmas. Nevertheless, our results provide some qualitative insight into the basic processes of MRI saturation, highlighting the importance of tearing-mode-like instabilities. Quantitative conclusions, as e.g., the scaling laws for the field strengths and the Maxwell stresses at MRI termination as a function of the initial magnetic field strength, should be taken with a grain of salt. These depend on the dissipative properties of the numerical scheme employed, which are likely to change when physical resistivity is considered.

6. The saturation phase of the MRI differs considerably between axisymmetric and unrestricted 3D models, and between models having a different initial field configuration. In $2 \mathrm{D}$ the flow does not break down into small-scale turbulence, instead the channel flows merge until they form one pair of large-scale coherent up- and down-flows. When the strength of the magnetic field exceeds $10^{15} \mathrm{G}$, the rotational profile is modified within a few tens of milliseconds.

7. Axisymmetric models having an initial magnetic field with a vanishing net flux through the computational box become turbulent after a growth phase dominated by channel flows. The saturation fields are considerably smaller than $10^{15} \mathrm{G}$.

8. The previous finding also holds for 3D models. Turbulence develops, but a spontaneous reorganization of the flow may lead to a re-appearance of channel modes, resulting in Maxwell stresses comparable to those found for axisymmetric models. In models which do not develop late-stage channel flows, field strengths up to several $10^{14} \mathrm{G}$ are encountered. The field is predominantly toroidal. The extent of the late-time channel activity depends on the development of secondary (parasitic) instabilities, both flow-driven (e.g., Kelvin-Helmholtz) and current-driven (e.g., tearing modes), which feed off the channel flows. The presence of these instabilities is determined to a large degree by the the aspect ratio of the computational box, i.e., we observe a strong dependence of the saturated state on the aspect ratio. For magnetorotational core collapse, our results suggest that secondary instabilities are fairly efficient in suppressing coherent channel flows during saturation.

9. For models having an initial entropy gradient, we find an important influence of convective stabilization or destabilization on the evolution of the MRI. We confirm the instability regimes predicted by our linear analysis with numerical simulations, the numerical growth rates being in accordance with the theoretical ones. The MRI is suppressed in convectively stable regions, the growth rates are reduced, and the geometry of the flow changes favoring radially less extended patterns. In the mixed regime, convectively unstable regions with comparably large entropy gradients are dominated by flows similar to volume-filling hydrodynamic convection. The magnetic field is expelled from convective cells and accumulates near the box boundaries. We note that the entropy gradients required for these effects are fairly shallow $\sim 0.1 \mathrm{~km}^{-1}$. We confirm the existence of the MBI regime for axisymmetric models, whereas the same models, computed in $3 \mathrm{D}$, experience the growth of non-axisymmetric modes.

10. In 3D models having a zero net magnetic flux we observe the development of large-scale coherent field patterns similar those seen by Lesur \& Ogilvie (2008), despite the turbulent nature of the velocity and magnetic fields. We also find differences in the flow patterns between MSI and mixed regime models. The tentative connection to a non-linear dynamo operating in the models remains to investigated further.

These results allow us to draw a few conclusions. Firstly, the MRI has the potential to play an important role for the dynamics of supernova explosions, at least for relatively fast rotating progenitors. The details of the evolution of the MRI depend crucially on the properties of the core, in particular its thermal stratification. This makes the study of the MRI in supernovae a subject of its own, related to the MRI in accretion discs but also quite different from it. Hence, there is a need for more investigations focusing on MRI properties specific to core collapse supernovae.

While local (or semi-global) simulations can yield interesting results regarding the physics of the MRI, several important aspects can only be addressed by global modeling. The detailed dependence of the geometry of the magnetic field at saturation, e.g., may depend strongly on the global dynamics and on the position of the box inside the core. Our simulations did not account for any of these factors: the background was in hydrostatic equilibrium, and we simulated models only in the equatorial region. Since the field geometry is of crucial importance for the global dynamics, e.g., for the generation of jet-like outflows in collapsars, conclusions on the dynamic influence of the MRI based on local simulations cannot be drawn easily. They require global models.

Additional investigations are also required to study the interplay of large-scale flows, e.g., the accretion of matter onto the proto-neutron star and the influence of neutrino radiation. Apart from modifying the regimes of the instability discussed here, the former process may allow for new instability regimes; in particular the combination of the MRI and the standing accretion shock instability may have interesting consequences for the dynamics of the explosion. While neutrino heating and cooling may, due to a net transport of entropy, lead to a stratification qualitatively similar to that of some models in this study with non-vanishing entropy gradients, it is too early to speculate on how neutrino radiation affects the MRI.

The inclusion of the MRI and its effects in global simulations requires a considerably careful treatment. The currently used approach of artificially enhancing the initial field strength by a constant factor is questionable. On the other hand, finding a better prescription relies on unraveling the dependence of saturated MRI driven turbulence on the different parameters of the system, as e.g., the rotation law, the thermodynamic conditions, and probably also the neutrino transport.

With our current simulations we are unfortunately not yet able to go beyond the stage of a qualitative proof of principle and to address important open questions of the MRI in core collapse supernovae. This would require additional 3D high-resolution non-ideal MHD simulations covering a large parameter space of possible rotation profiles, thermal stratifications, and magnetic field geometries. We are planning to address these issues in future work.

Acknowledgements. This research has been supported by the Spanish Ministerio de Educación y Ciencia (grants AYA2007-67626-C03-01, CSD2007-00050), and by the Collaborative Research Center on Gravitational Wave Astronomy 


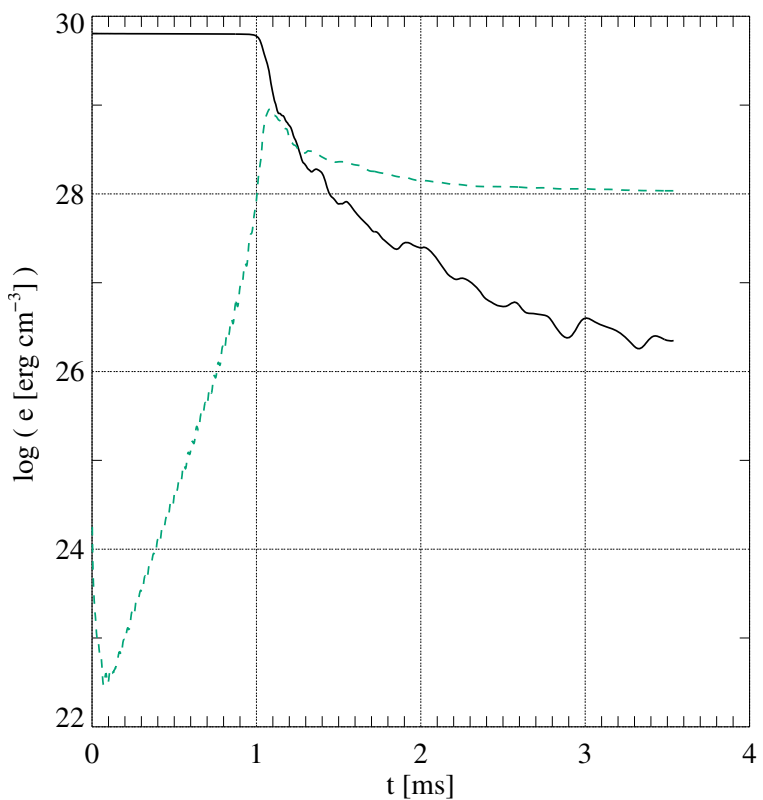

Fig. A.1. Evolution of the magnetic energy density of a current-sheet model simulated on a square computational grid (edge length $0.25 \mathrm{~km}$; $200 \times 200$ zones). The model has an initial density $\rho_{0}=2.5 \times 10^{13} \mathrm{~g} \mathrm{~cm}^{-3}$, and an initial field strength $b_{0}^{x}=1.6 \times 10^{15} \mathrm{G}$. The wavelength of the initially sinusoidally varying magnetic field is $a=31.25 \mathrm{~m}$. The black solid line and the green dashed line show the magnetic energy density corresponding to the $x$ and the $y$ component of the magnetic field, respectively.

of the Deutsche Forschungsgemeinschaft (DFG SFB/Transregio 7). MAA is a Ramón y Cajal fellow of the Ministerio de Educación y Ciencia. Most of the simulations were performed at the Rechenzentrum Garching (RZG) of the MaxPlanck-Society. We are also thankful for the computer resources, the technical expertise, and the assistance provided by the Barcelona Supercomputing Center - Centro Nacional de Supercomputación. Finally, we would like to acknowledge enlightening discussions with Tom Abel and Fen Zhao about the MRI.

\section{Appendix A: Growth rates of resistive instabilities}

Our simulations of the MRI indicate that the termination of the growth of the instability is determined, at least partially, by resistive instabilities of the tearing-mode type. Although there exist detailed investigations of this kind of resistive instabilities (see, e.g., Biskamp 2000), the application of the results to our study is hampered by the completely different type of dissipative effects we are facing here: all previous results hold for instabilities due to physical resistivity, whereas our ideal MHD simulations are affected by numerical resistivity, only. Hence, we had to determine the growth rates of resistive instabilities from numerical experiments without referring to analytic results - although, as we will see, there exist certain similarities.

We simulated the evolution of two-dimensional current-sheet models on a Cartesian grid $\left[x_{0}, x_{1}\right] \times\left[y_{0}, y_{1}\right]$ with $m_{x} \times m_{y}$ zones imposing periodic boundary conditions. The fluid is described by an ideal-gas equation of state with an adiabatic index $\Gamma=4 / 3$. We used initial data mimicking MRI-generated channel flows: the initial magnetic field varies sinusoidally in a gas of constant density $\rho_{0}$ and pressure $P_{0}$,

$$
\begin{aligned}
& b^{x}=b_{0}^{x} \sin \frac{2 \pi\left(y-y_{0}\right)}{a}, \\
& b^{z}=-b_{0}^{x},
\end{aligned}
$$

Table A.1. Parameters of the 2D current sheets simulated to determine the growth rates of resistive instabilities. The columns give from left to right the edge length of the square simulation box, the number of grid zones per dimension, the initial density (in units of $10^{13} \mathrm{~g} / \mathrm{cm}^{-3}$ ), the sound speed (in units of $10^{9} \mathrm{~cm} / \mathrm{s}$ ), the magnetic field (in units of $\left.10^{14} \mathrm{G}\right)$, the wavelength of the initial field, and the growth rate,

\begin{tabular}{|c|c|c|c|c|c|c|}
\hline $\begin{array}{c}L \\
{[\mathrm{~km}]}\end{array}$ & $m_{x}$ & $\begin{array}{c}\rho_{0 ; 13} \\
{\left[\mathrm{~g} \mathrm{~cm}^{-3}\right]}\end{array}$ & $\begin{array}{c}c_{\mathrm{S} ; 9} \\
{\left[\mathrm{~cm} \mathrm{~s}^{-1}\right]}\end{array}$ & $\begin{array}{c}b_{0 ; 14}^{x} \\
{[\mathrm{G}]}\end{array}$ & $\begin{array}{c}a \\
{[\mathrm{~m}]}\end{array}$ & $\begin{array}{c}\sigma \\
{\left[\mathrm{ms}^{-1}\right]}\end{array}$ \\
\hline 0.25 & 50 & 2.5 & 3.1 & 8 & 125 & 0.45 \\
\hline 0.25 & 50 & 2.5 & 3.1 & 16 & 125 & 1.6 \\
\hline 0.25 & 50 & 2.5 & 3.1 & 32 & 125 & 6.0 \\
\hline 0.25 & 50 & 2.5 & 3.1 & 64 & 125 & 32 \\
\hline 0.25 & 50 & 2.5 & 3.1 & 16 & 62.5 & 10 \\
\hline 0.25 & 50 & 2.5 & 3.1 & 32 & 62.5 & 23 \\
\hline 0.25 & 100 & 2.5 & 3.1 & 16 & 125 & 0.60 \\
\hline 0.25 & 100 & 2.5 & 3.1 & 32 & 125 & 3.1 \\
\hline 0.25 & 100 & 2.5 & 3.1 & 64 & 125 & 12 \\
\hline 0.25 & 100 & 2.5 & 3.1 & 128 & 125 & 43 \\
\hline 0.25 & 100 & 2.5 & 3.1 & 16 & 62.5 & 3.7 \\
\hline 0.25 & 100 & 2.5 & 3.1 & 32 & 62.5 & 14 \\
\hline 0.25 & 100 & 2.5 & 3.1 & 64 & 62.5 & 50 \\
\hline 0.25 & 100 & 2.5 & 3.1 & 128 & 62.5 & 110 \\
\hline 0.25 & 100 & 0.025 & 1.4 & 3.2 & 62.5 & 25 \\
\hline 0.25 & 100 & 0.025 & 3.1 & 3.2 & 62.5 & 15 \\
\hline 0.25 & 100 & 0.025 & 6.1 & 3.2 & 62.5 & 8.0 \\
\hline 0.25 & 100 & 2.5 & 1.4 & 32 & 62.5 & 25 \\
\hline 0.25 & 100 & 2.5 & 3.1 & 32 & 62.5 & 14 \\
\hline 0.25 & 100 & 2.5 & 1.4 & 6.1 & 62.5 & 7.8 \\
\hline 0.25 & 100 & 250 & 6.6 & 320 & 62.5 & 7.4 \\
\hline 0.25 & 100 & 250 & 3.1 & 320 & 62.5 & 15 \\
\hline 0.25 & 100 & 2.5 & 12.4 & 64 & 62.5 & 16 \\
\hline 0.25 & 100 & 2.5 & 3.1 & 4 & 31.25 & 1.2 \\
\hline 0.25 & 100 & 2.5 & 3.1 & 8 & 31.25 & 5.9 \\
\hline 0.25 & 100 & 2.5 & 3.1 & 16 & 31.25 & 20 \\
\hline 0.25 & 100 & 2.5 & 3.1 & 32 & 31.25 & 53 \\
\hline 0.25 & 100 & 2.5 & 3.1 & 64 & 31.25 & 110 \\
\hline 0.25 & 200 & 2.5 & 3.1 & 32 & 125 & 0.9 \\
\hline 0.25 & 200 & 2.5 & 3.1 & 64 & 125 & 4.6 \\
\hline 0.25 & 200 & 2.5 & 3.1 & 128 & 125 & 19 \\
\hline 0.25 & 200 & 2.5 & 3.1 & 32 & 62.5 & 5.4 \\
\hline 0.25 & 200 & 2.5 & 3.1 & 64 & 62.5 & 24 \\
\hline 0.25 & 200 & 2.5 & 3.1 & 128 & 62.5 & 76 \\
\hline 0.25 & 200 & 2.5 & 3.1 & 16 & 31.25 & 7.9 \\
\hline 0.25 & 200 & 2.5 & 3.1 & 32 & 31.25 & 28 \\
\hline 0.25 & 200 & 2.5 & 3.1 & 64 & 31.25 & 95 \\
\hline 0.25 & 200 & 2.5 & 3.1 & 128 & 31.25 & 250 \\
\hline 0.25 & 200 & 2.5 & 3.1 & 4 & 15.625 & 2.5 \\
\hline 0.25 & 200 & 2.5 & 3.1 & 16 & 15.625 & 40 \\
\hline 0.25 & 200 & 2.5 & 3.1 & 64 & 15.625 & 260 \\
\hline 0.5 & 100 & 2.5 & 3.1 & 32 & 250 & 1.2 \\
\hline 0.5 & 100 & 2.5 & 3.1 & 64 & 250 & 5.6 \\
\hline 0.5 & 100 & 2.5 & 3.1 & 128 & 250 & 20 \\
\hline 0.5 & 100 & 2.5 & 3.1 & 16 & 125 & 1.9 \\
\hline 0.5 & 100 & 2.5 & 3.1 & 32 & 125 & 7.5 \\
\hline 0.5 & 100 & 2.5 & 3.1 & 64 & 125 & 26 \\
\hline 0.5 & 100 & 2.5 & 3.1 & 8 & 62.5 & 3.0 \\
\hline 0.5 & 100 & 2.5 & 3.1 & 16 & 62.5 & 10 \\
\hline 0.5 & 100 & 2.5 & 3.1 & 32 & 62.5 & 24 \\
\hline 0.5 & 100 & 2.5 & 3.1 & 64 & 62.5 & 61 \\
\hline
\end{tabular}
respectively. 

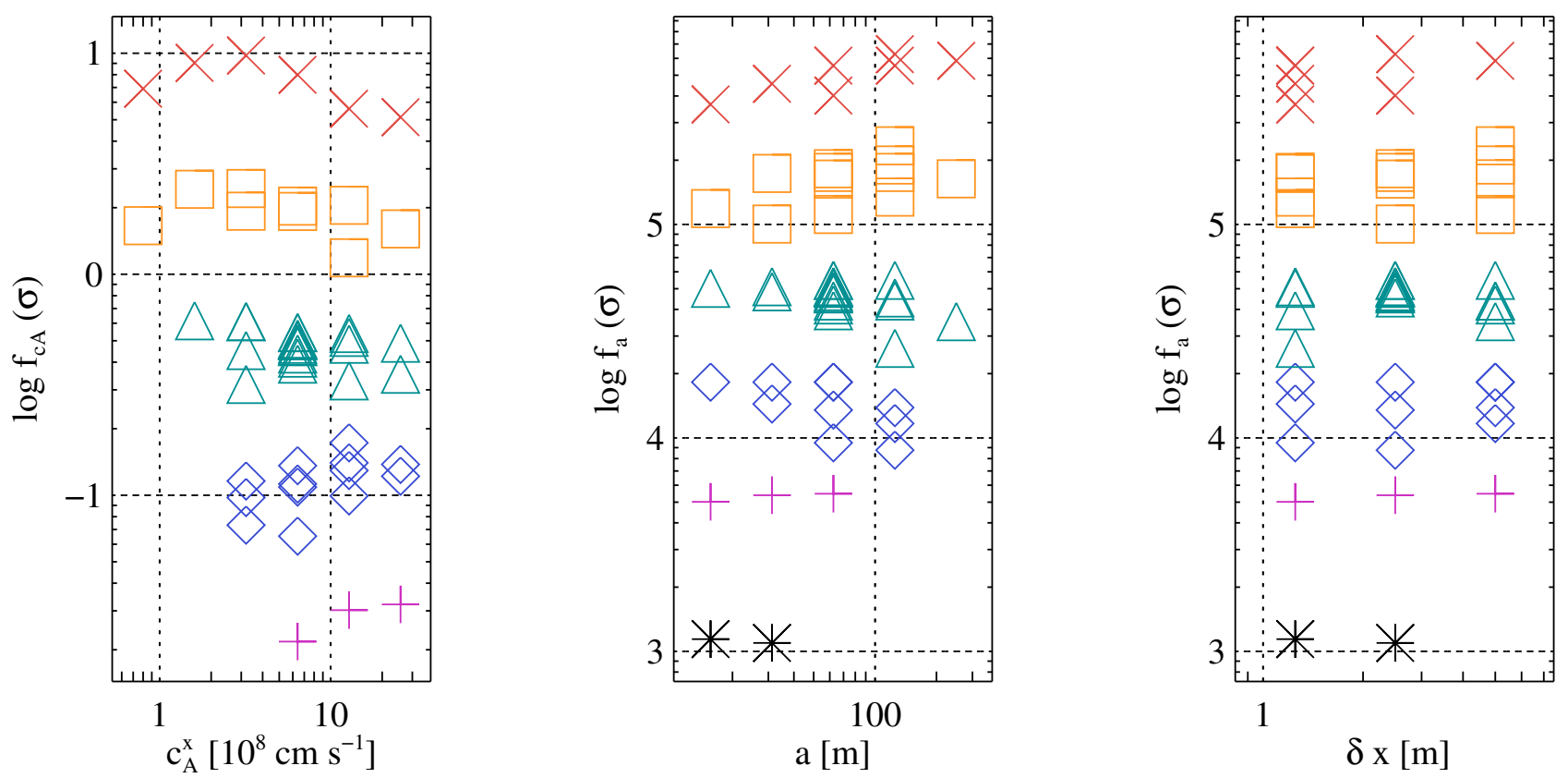

Fig. A.2. Dependence of the growth rate of resistive instabilities, $\sigma$, on various parameters of $2 \mathrm{D}$ current sheet models. The panels show $f_{\mathrm{cA}}$ as a function of the initial Alfvén velocity $\left(c_{\mathrm{A}}^{x}\right.$; left panel), and $f_{a}$ as a function of the width of the current sheet ( $a$; middle panel) and of the grid resolution ( $\delta x$; right panel). The left panel shows groups of models with different $a: 250 \mathrm{~m}$ (pink plus sign), $125 \mathrm{~m}$ (blue diamond), $62.5 \mathrm{~m}$ (green triangle), $32.25 \mathrm{~m}$ (orange square), and $15.625 \mathrm{~m}$ (red cross). The other two panels show groups of models with different Alfvén velocity (in units of $10^{8} \mathrm{~cm} \mathrm{~s}^{-1}$ ): 0.8 (black asterisk), 1.6 (pink plus sign), 3.2 (blue diamond), 6.4 (green triangle), 12.8 (orange square), and 25.6 (red cross) respectively.

and having a velocity in $x$-direction given by

$v^{x}=v_{0}^{x} \sin \frac{2 \pi\left(y-y_{0}\right)-\pi / 2}{a}$.

Here, $a$ denotes the initial width of the flux sheet, and $v_{0}^{x}$ is equal to one half of the Alfvén velocity $c_{\mathrm{A}}^{x}$ corresponding to $b_{0}^{x}$. The presence of the (shear-free!) initial $x$-velocity is not essential, as it changes the growth rates of the instability only little. However, as we observe this kind of a velocity in channel flows, we have included it in our simulations.

We perturbed the sheet by a small random $y$-velocity $\left(10^{-2} \times\right.$ $\left.c_{\mathrm{A}}^{x}\right)$. The parameters of the model are chosen to mimic the situations encountered in MRI simulations (see Table A.1). To isolate the dependence of the growth rates on the physical and numerical parameters, we varied the initial magnetic field strength, $b_{0}^{x}$, the initial density, $\rho_{0}$, the width of the current sheet, $a$, and the grid resolution, $\delta x=\left(x_{1}-x_{0}\right) / m_{x}$. We chose the grid resolution such that the current sheet is covered by 12 to 100 zones. The initial pressure is $P_{0}=\kappa \rho_{0}^{\Gamma}$.

We show one typical result for the evolution of our models in Fig. A.1. After a short initial phase, the transverse magnetic energy density (green line) grows roughly exponentially as tearing modes develop. Initially the growth rate is approximately constant, but it increases by a factor of $\sim 4$ towards saturation. Simultaneously, the $x$-component of the magnetic field decreases strongly until it is of similar strength as the $y$-component. At this point, the coherent current sheets are completely disrupted by the resistive instability.

We determined estimates of the growth rates of the resistive instability using the time derivative of the transverse magnetic energy density, i.e., the time derivative of the magnetic energy density corresponding to the $y$-component of the magnetic field. To find a scaling relation of the form

$\sigma \propto\left(c_{\mathrm{A}}^{x}\right)^{\gamma_{\mathrm{A}}}\left(c_{\mathrm{S}}\right)^{\gamma_{\mathrm{S}}}(\delta x)^{\gamma_{\delta}}(a)^{\gamma_{a}}$ we define the following functions of the growth rate $\sigma$,

$$
\begin{aligned}
f_{c A} & =\sigma\left(c_{\mathrm{A}}^{x}\right)^{-\gamma_{\mathrm{A}}}\left(c_{\mathrm{S}}\right)^{-\gamma_{\mathrm{S}}}(\delta x)^{-\gamma_{\delta}}, \\
f_{a} & =\sigma\left(c_{\mathrm{S}}\right)^{-\gamma_{\mathrm{S}}}(a)^{-\gamma_{a}}(\delta x)^{-\gamma_{\delta}},
\end{aligned}
$$

and adjust the exponents $\gamma_{\mathrm{A}}, \gamma_{\mathrm{S}}, \gamma_{a}$, and $\gamma_{\delta}$ to determine the scaling of $\sigma$ with the respective parameters. Our preferred set of scaling exponents is

$\gamma_{\mathrm{A}}=1.75$

$\gamma_{\mathrm{S}}=-0.75$

$\gamma_{a}=-2$

$\gamma_{\delta}=1$

which implies the following scaling law:

$\sigma \propto\left(\frac{c_{\mathrm{A}}}{c_{\mathrm{S}}}\right)^{0.75}\left(\frac{c_{\mathrm{A}} \delta x}{a^{2}}\right)$.

We demonstrate the quality of the fit parameters in Fig. A.2 showing $f_{\mathrm{cA}}$ as a function of the initial Alfvén velocity (left panel), and $f_{a}$ as a function of the width of the current sheet (middle panel) and of the grid resolution (right panel). None of the groups of models representing the variation of one parameter (distinguished by a different color and symbol in the figure) exhibits a strong trend with $c_{A}, a$ or $\delta x$, i.e., our scaling exponents provide an adequate fit to the data.

Due to a relatively large scatter in the growth rates, the scaling relation, Eq. (A.4), should not be taken too literally. We note, however, that our computed rates are compatible with those of the resistive instabilities (for fields of similar strength) in MRI models, i.e., approximately one millisecond for $b_{0}^{x} \sim 10^{15} \mathrm{G}$. An additional dependence of $\sigma$ on the domain size, $L$, cannot be excluded, but we did not examine this possibility any further. For small $a$ and coarse grids our scaling formula tends to overestimate the growth rate for large initial Alfvén velocities, and 
Table B.1. List of 2D models having a positive entropy gradient and an initial rotation profile given by $\Omega_{0}=1900 \mathrm{~s}^{-1}$ and $\alpha_{\Omega}=-1.25$. The columns give (from left to right) the number of grid zones $m_{\varpi} \times m_{z}$ (the box has an edge length $L_{\varpi}=L_{z}=1 \mathrm{~km}$ ), the type of boundary condition which was applied (d: velocity damping; p: periodic), the adiabatic index of the gas, $\Gamma$ (models computed with an ideal-gas equation of state instead of the hybrid one are marked "id"), the initial entropy, $S_{0}$, and the radial entropy gradient, $\partial_{\varpi} S$. The next three columns give the parameter $C$ determining the instability regime (see Eq. (24)), the theoretical growth rate, $\sigma_{\text {th }}$, and the strength of the initially uniform magnetic field, $b_{0}^{z}$. The last two columns list the numerical growth rate, $\sigma$, and the value of the Maxwell stress component $M_{\varpi \phi}^{\text {term }}$ at MRI termination.

\begin{tabular}{|c|c|c|c|c|c|c|c|c|c|}
\hline $\bar{m}_{\varpi} \times m_{z}$ & $\overline{\mathrm{BC}}$ & $\overline{\bar{\Gamma}}$ & $\overline{\bar{S} S_{0}}$ & $\begin{array}{c}\partial_{\varpi} S \\
{\left[\mathrm{~km}^{-1}\right]}\end{array}$ & $\overline{\bar{C}}$ & $\begin{array}{c}\sigma_{\text {th }} \\
{\left[\mathrm{ms}^{-1}\right.}\end{array}$ & $\begin{array}{c}b_{0}^{z} \\
{\left[10^{12} \mathrm{G}\right.}\end{array}$ & $\begin{array}{c}\sigma \\
{\left[\mathrm{ms}^{-1}\right]}\end{array}$ & $\begin{array}{c}M_{\varpi \phi}^{\text {term }} \\
{\left[10^{28} \mathrm{G}^{2} \mathrm{~cm}^{-3}\right]}\end{array}$ \\
\hline $100 \times 100$ & $\mathrm{~d}$ & 1.31 & 0.20 & 0.020 & -1.7 & 0.73 & 10 & 0.72 & 1.1 \\
\hline $100 \times 100$ & d & 1.31 & 0.20 & 0.020 & -1.7 & 0.73 & 20 & 0.71 & 1.3 \\
\hline $100 \times 100$ & d & 1.31 & 0.20 & 0.020 & -1.7 & 0.73 & 40 & 0.70 & 1.6 \\
\hline $100 \times 100$ & d & 1.31 & 0.20 & 0.020 & -1.7 & 0.73 & 80 & 0.60 & 1.8 \\
\hline $200 \times 200$ & d & 1.31 & 0.20 & 0.020 & -1.7 & 0.73 & 10 & 0.71 & 1.3 \\
\hline $400 \times 400$ & $\mathrm{~d}$ & 1.31 & 0.20 & 0.020 & -1.7 & 0.73 & 10 & 0.67 & 1.2 \\
\hline $400 \times 400$ & d & 1.31 & 0.20 & 0.020 & -1.7 & 0.73 & 20 & 0.69 & 1.5 \\
\hline $50 \times 50$ & d & $5 / 3$ & 0.20 & 0.040 & -1.1 & 0.49 & 10 & 0.46 & 0.97 \\
\hline $50 \times 50$ & d & $5 / 3$ & 0.20 & 0.040 & -1.1 & 0.49 & 20 & 0.61 & 1.7 \\
\hline $50 \times 50$ & d & $5 / 3$ & 0.20 & 0.040 & -1.1 & 0.49 & 40 & 0.48 & 0.87 \\
\hline $50 \times 50$ & d & $5 / 3$ & 0.20 & 0.040 & -1.1 & 0.49 & 80 & 0.39 & 0.93 \\
\hline $50 \times 50$ & d & $1.31, \mathrm{id}$ & 0.20 & 0.040 & -0.69 & 0.32 & 20 & 0.50 & 0.53 \\
\hline $50 \times 50$ & d & $1.31, \mathrm{id}$ & 0.20 & 0.040 & -0.69 & 0.32 & 40 & 0.30 & 0.27 \\
\hline $50 \times 50$ & d & $1.31, \mathrm{id}$ & 0.20 & 0.040 & -0.69 & 0.32 & 80 & 0.28 & 0.22 \\
\hline $50 \times 50$ & d & 1.31 & 0.20 & 0.040 & -0.69 & 0.32 & 4 & 0.25 & 0.21 \\
\hline $50 \times 50$ & d & 1.31 & 0.20 & 0.040 & -0.69 & 0.32 & 20 & 0.49 & 0.50 \\
\hline $50 \times 50$ & d & 1.31 & 0.20 & 0.040 & -0.69 & 0.32 & 40 & 0.28 & 0.24 \\
\hline $50 \times 50$ & d & 1.31 & 0.20 & 0.040 & -0.69 & 0.32 & 80 & 0.28 & 0.22 \\
\hline $100 \times 100$ & d & 1.31 & 0.20 & 0.040 & -0.69 & 0.32 & 10 & 0.49 & 0.55 \\
\hline $100 \times 100$ & d & 1.31 & 0.20 & 0.040 & -0.69 & 0.32 & 20 & 0.30 & 0.29 \\
\hline $100 \times 100$ & d & 1.31 & 0.20 & 0.040 & -0.69 & 0.32 & 40 & 0.30 & 0.29 \\
\hline $100 \times 100$ & d & 1.31 & 0.20 & 0.040 & -0.69 & 0.32 & 80 & 0.28 & 0.22 \\
\hline $200 \times 200$ & d & 1.31 & 0.20 & 0.040 & -0.69 & 0.32 & 4 & 0.53 & 0.40 \\
\hline $200 \times 200$ & d & 1.31 & 0.20 & 0.040 & -0.69 & 0.32 & 10 & 0.30 & 0.20 \\
\hline $200 \times 200$ & d & 1.31 & 0.20 & 0.040 & -0.69 & 0.32 & 20 & 0.30 & 0.22 \\
\hline $200 \times 200$ & d & 1.31 & 0.20 & 0.040 & -0.69 & 0.32 & 40 & 0.30 & 0.30 \\
\hline $200 \times 200$ & d & 1.31 & 0.20 & 0.040 & -0.69 & 0.32 & 80 & 0.26 & 0.18 \\
\hline $100 \times 100$ & d & 1.31 & 0.20 & 0.080 & 1.1 & 0 & 20 & $<0.01$ & \\
\hline $200 \times 200$ & d & 1.31 & 0.20 & 0.080 & 1.1 & 0 & 10 & $<0.05$ & \\
\hline $100 \times 100$ & $\mathrm{p}$ & $5 / 3$ & 0.20 & 0.020 & -1.8 & 0.82 & 20 & 0.79 & 4.9 \\
\hline $100 \times 100$ & $\mathrm{p}$ & 1.31 & 0.20 & 0.020 & -1.7 & 0.73 & 10 & 0.73 & 1.9 \\
\hline $100 \times 100$ & $\mathrm{p}$ & 1.31 & 0.20 & 0.020 & -1.7 & 0.73 & 20 & 0.71 & 33 \\
\hline $100 \times 100$ & $\mathrm{p}$ & 1.31 & 0.20 & 0.020 & -1.7 & 0.73 & 40 & 0.72 & 202 \\
\hline $100 \times 100$ & $\mathrm{p}$ & 1.31 & 0.20 & 0.020 & -1.7 & 0.73 & 80 & 0.62 & 411 \\
\hline $50 \times 50$ & $\mathrm{p}$ & 1.31 & 0.20 & 0.040 & -0.69 & 0.32 & 20 & 0.51 & 1.7 \\
\hline $50 \times 50$ & $\mathrm{p}$ & 1.31 & 0.20 & 0.040 & -0.69 & 0.32 & 40 & 0.31 & 90 \\
\hline $50 \times 50$ & $\mathrm{p}$ & 1.31 & 0.20 & 0.040 & -0.69 & 0.32 & 80 & 0.32 & 159 \\
\hline $100 \times 100$ & $\mathrm{p}$ & 1.31 & 0.20 & 0.040 & -0.69 & 0.32 & 10 & 0.53 & 0.64 \\
\hline $100 \times 100$ & $\mathrm{p}$ & 1.31 & 0.20 & 0.040 & -0.69 & 0.32 & 20 & 0.30 & 6.8 \\
\hline $100 \times 100$ & $\mathrm{p}$ & 1.31 & 0.20 & 0.040 & -0.69 & 0.32 & 40 & 0.30 & 98 \\
\hline $100 \times 100$ & $\mathrm{p}$ & 1.31 & 0.20 & 0.040 & -0.69 & 0.32 & 80 & 0.31 & 201 \\
\hline $50 \times 50$ & $\mathrm{p}$ & $5 / 3$ & 0.20 & 0.040 & -1.1 & 0.49 & 10 & 0.45 & 0.55 \\
\hline $50 \times 50$ & $\mathrm{p}$ & $5 / 3$ & 0.20 & 0.040 & -1.1 & 0.49 & 20 & 0.59 & 3.1 \\
\hline $50 \times 50$ & $\mathrm{p}$ & $5 / 3$ & 0.20 & 0.040 & -1.1 & 0.49 & 40 & 0.47 & 201 \\
\hline $50 \times 50$ & $\mathrm{p}$ & $5 / 3$ & 0.20 & 0.040 & -1.1 & 0.49 & 80 & 0.38 & 139 \\
\hline $100 \times 100$ & $\mathrm{p}$ & $5 / 3$ & 0.20 & 0.040 & -1.1 & 0.49 & 4 & 0.40 & 0.43 \\
\hline $100 \times 100$ & $\mathrm{p}$ & $5 / 3$ & 0.20 & 0.040 & -1.1 & 0.49 & 10 & 0.63 & 0.83 \\
\hline $100 \times 100$ & $\mathrm{p}$ & $5 / 3$ & 0.20 & 0.040 & -1.1 & 0.49 & 20 & 0.49 & 5.0 \\
\hline $100 \times 100$ & $\mathrm{p}$ & $5 / 3$ & 0.20 & 0.040 & -1.1 & 0.49 & 40 & 0.47 & 262 \\
\hline $100 \times 100$ & $\mathrm{p}$ & $5 / 3$ & 0.20 & 0.040 & -1.1 & 0.49 & 80 & 0.45 & 978 \\
\hline
\end{tabular}

for sound speeds much larger than the Alfvén velocity the rates depend more strongly on the sound speed than predicted by our formula. Because both situations do not apply to our MRI models, we did not pursue these issues any further. Our scaling law loses its validity, if the Alfvén velocity exceeds the sound speed. Thus, we excluded two respective models in the derivation of our scaling relation.
Bearing in mind the uncertainties regarding the physical meaning of a purely numerical resistivity and the precise values of the scaling we may try to interpret our result summarized in Eq. (A.11). As the product of the Alfvén velocity and the grid spacing, $c_{A} \delta x$, defines an effective resistivity, we may conclude that the growth time of the instability is set by the time 
Table B.2. List of 2D models having a negative initial entropy gradient. The columns give (from left to right) the number of grid zones $m_{\varpi} \times m_{z}$ (the box has an edge length $L_{\sigma}=L_{z}=1 \mathrm{~km}$ ), the type of boundary condition which was applied (d: velocity damping; p: periodic), the rotation law ("d": differential rotation with $\Omega_{0}=1900 \mathrm{~s}^{-1}$ and $\alpha_{\Omega}=-1.25$; " $r$ " rigid rotation with an angular velocity of $\Omega$ ), the adiabatic index of the gas, $\Gamma$, the initial entropy, $S_{0}$, the radial entropy gradient, $\partial_{\varpi} S$, and the strength of the initially uniform magnetic field, $b_{0}^{z}$. The next columns give the three quantities $\mathcal{R}_{\varpi} / \Omega_{0}^{2}, N^{2} / \Omega_{0}^{2}$ and $C$ determining the instability regime (see Eq. (24)), followed by the theoretical growth rate, $\sigma_{\text {th }}$, and the type of the instability. The last two columns list the numerical growth rate, $\sigma$, and the value of the Maxwell stress component $M_{\varpi \phi}^{\text {term }}$ at MRI termination.

\begin{tabular}{|c|c|c|c|c|c|c|c|c|c|c|c|c|c|}
\hline$\overline{m_{\varpi} \times m_{z}}$ & $\overline{\mathrm{BC}}$ & Rot & $\bar{\Gamma}$ & 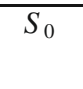 & $\begin{array}{c}\partial_{\varpi} S \\
{\left[\mathrm{~km}^{-1}\right]}\end{array}$ & $\begin{array}{l}b_{0}^{z} \\
{\left[10^{12} \mathrm{G}\right]}\end{array}$ & $\overline{\mathcal{R}_{\varpi} / \Omega_{0}^{2}}$ & $\overline{N^{2} / \Omega_{0}^{2}}$ & $\bar{C}$ & $\begin{array}{c}\sigma_{\text {th }} \\
{\left[\mathrm{ms}^{-1}\right.}\end{array}$ & regime & $\begin{array}{c}\sigma \\
{\left[\mathrm{ms}^{-1}\right]}\end{array}$ & $\begin{array}{c}M_{\varpi \phi}^{\text {term }} \\
{\left[10^{28} \mathrm{G}^{2} \mathrm{~cm}^{-3}\right]}\end{array}$ \\
\hline $100 \times 100$ & $\mathrm{~d}$ & $\mathrm{~d}$ & 1.31 & 0.20 & -0.019 & 0 & -2.5 & -0.90 & -3.4 & 1.6 & $\operatorname{mix}$ & 0 & 0 \\
\hline $100 \times 100$ & d & d & 1.31 & 0.20 & -0.019 & 10 & -2.5 & -0.90 & -3.4 & 1.6 & $\operatorname{mix}$ & 1.2 & 2.6 \\
\hline $100 \times 100$ & $\mathrm{~d}$ & $\mathrm{~d}$ & 1.31 & 0.20 & -0.019 & 20 & -2.5 & -0.90 & -3.4 & 1.6 & $\operatorname{mix}$ & 1.3 & 3.4 \\
\hline $100 \times 100$ & d & d & 1.31 & 0.20 & -0.019 & 40 & -2.5 & -0.90 & -3.4 & 1.6 & $\operatorname{mix}$ & 1.4 & 5.7 \\
\hline $100 \times 100$ & d & d & 1.31 & 0.20 & -0.019 & 80 & -2.5 & -0.90 & -3.4 & 1.6 & $\operatorname{mix}$ & 1.3 & 6.0 \\
\hline $50 \times 50$ & $\mathrm{~d}$ & d & 1.31 & 0.20 & -0.038 & 20 & -2.5 & -1.8 & -4.3 & 2.0 & $\operatorname{mix}$ & 1.6 & 4.2 \\
\hline $100 \times 100$ & d & d & 1.31 & 0.20 & -0.038 & 0 & -2.5 & -1.8 & -4.3 & 2.0 & $\operatorname{mix}$ & 0 & 0 \\
\hline $100 \times 100$ & d & d & 1.31 & 0.20 & -0.038 & 10 & -2.5 & -1.8 & -4.3 & 2.0 & $\operatorname{mix}$ & 1.5 & 3.3 \\
\hline $100 \times 100$ & d & d & 1.31 & 0.20 & -0.038 & 20 & -2.5 & -1.8 & -4.3 & 2.0 & $\operatorname{mix}$ & 1.7 & 5.3 \\
\hline $100 \times 100$ & d & d & 1.31 & 0.20 & -0.038 & 40 & -2.5 & -1.8 & -4.3 & 2.0 & $\operatorname{mix}$ & 1.7 & 7.8 \\
\hline $100 \times 100$ & d & d & 1.31 & 0.20 & -0.038 & 80 & -2.5 & -1.8 & -4.3 & 2.0 & $\operatorname{mix}$ & 1.7 & 9.5 \\
\hline $100 \times 100$ & d & d & 1.31 & 0.20 & -0.075 & 0 & -2.5 & -3.6 & -6.1 & 2.8 & $\operatorname{mix}$ & 0 & 0 \\
\hline $100 \times 100$ & d & d & 1.31 & 0.20 & -0.075 & 10 & -2.5 & -3.6 & -6.1 & 2.8 & $\operatorname{mix}$ & 2.4 & 1.3 \\
\hline $100 \times 100$ & d & d & 1.31 & 0.20 & -0.075 & 20 & -2.5 & -3.6 & -6.1 & 2.8 & $\operatorname{mix}$ & 2.6 & 4.8 \\
\hline $100 \times 100$ & d & d & 1.31 & 0.20 & -0.075 & 40 & -2.5 & -3.6 & -6.1 & 2.8 & $\operatorname{mix}$ & 2.5 & 9.7 \\
\hline $100 \times 100$ & d & d & 1.31 & 0.20 & -0.15 & 0 & -2.5 & -7.2 & -9.7 & 4.4 & conv & 3.0 & 0 \\
\hline $100 \times 100$ & d & d & 1.31 & 0.20 & -0.15 & 20 & -2.5 & -7.2 & -9.7 & 4.4 & conv & 4.0 & 1.2 \\
\hline $100 \times 100$ & d & d & 1.31 & 0.20 & -0.15 & 40 & -2.5 & -7.2 & -9.7 & 4.4 & conv & 3.8 & 3.7 \\
\hline $100 \times 100$ & $\mathrm{p}$ & d & $5 / 3$ & 0.20 & -0.019 & 20 & -2.5 & -0.70 & -3.2 & 1.5 & $\operatorname{mix}$ & 1.3 & 6.0 \\
\hline $100 \times 100$ & $\mathrm{p}$ & d & $5 / 3$ & 0.20 & -0.019 & 40 & -2.5 & -0.70 & -3.2 & 1.5 & $\operatorname{mix}$ & 1.4 & 50 \\
\hline $50 \times 50$ & $\mathrm{p}$ & d & 1.31 & 0.20 & -0.038 & 20 & -2.5 & -1.8 & -4.3 & 2.0 & $\operatorname{mix}$ & 1.6 & 12 \\
\hline $50 \times 50$ & $\mathrm{p}$ & d & 1.31 & 0.20 & -0.038 & 40 & -2.5 & -1.8 & -4.3 & 2.0 & $\operatorname{mix}$ & 1.7 & 19.5 \\
\hline $100 \times 100$ & $\mathrm{p}$ & d & 1.31 & 0.20 & -0.038 & 20 & -2.5 & -1.8 & -4.3 & 2.0 & $\operatorname{mix}$ & 1.7 & 27 \\
\hline $100 \times 100$ & $\mathrm{p}$ & d & 1.31 & 0.20 & -0.038 & 40 & -2.5 & -1.8 & -4.3 & 2.0 & $\operatorname{mix}$ & 1.7 & 22 \\
\hline $100 \times 100$ & d & $r^{1000}$ & 1.31 & 0.20 & -0.075 & 0 & 0 & -14 & -14 & 3.4 & conv & 2.7 & \\
\hline $100 \times 100$ & d & $r^{1500}$ & 1.31 & 0.20 & -0.075 & 0 & 0 & -5.7 & -5.7 & 2.1 & conv & 1.6 & \\
\hline $100 \times 100$ & d & $r^{1900}$ & 1.31 & 0.20 & -0.075 & 0 & 0 & -3.2 & -3.2 & 1.5 & MBI & 0 & \\
\hline $100 \times 100$ & d & $r^{1500}$ & 1.31 & 0.20 & -0.075 & $10^{-8}$ & 0 & -5.7 & -5.7 & 2.1 & conv & 1.5 & $1.4 \times 10^{-18}$ \\
\hline $100 \times 100$ & d & $r^{1500}$ & 1.31 & 0.20 & -0.075 & 1 & 0 & -5.7 & -5.7 & 2.1 & conv & 1.5 & 0.013 \\
\hline $100 \times 100$ & d & $r^{1500}$ & 1.31 & 0.20 & -0.075 & 10 & 0 & -5.7 & -5.7 & 2.1 & conv & 1.8 & 1.7 \\
\hline $100 \times 100$ & d & $r^{1900}$ & 1.31 & 0.40 & -0.10 & 4 & 0 & -3.6 & -3.6 & 1.7 & MBI & 1.1 & 0.22 \\
\hline $100 \times 100$ & d & $r^{1900}$ & 1.31 & 0.40 & -0.10 & 8 & 0 & -3.6 & -3.6 & 1.7 & MBI & 1.2 & 1.6 \\
\hline $100 \times 100$ & d & $r^{1900}$ & 1.31 & 0.40 & -0.10 & 10 & 0 & -3.6 & -3.6 & 1.7 & MBI & 1.1 & 1.6 \\
\hline $100 \times 100$ & d & $r^{1900}$ & 1.31 & 0.40 & -0.10 & 20 & 0 & -3.6 & -3.6 & 1.7 & MBI & 1.4 & 5.2 \\
\hline $100 \times 100$ & d & $r^{1900}$ & 1.31 & 0.40 & -0.10 & 40 & 0 & -3.6 & -3.6 & 1.7 & MBI & & \\
\hline $200 \times 200$ & d & $r^{1900}$ & 1.31 & 0.40 & -0.10 & 0 & 0 & -3.6 & -3.6 & 1.7 & MBI & 0 & 0 \\
\hline $200 \times 200$ & d & $r^{1900}$ & 1.31 & 0.40 & -0.10 & 0.01 & 0 & -3.6 & -3.6 & 1.7 & MBI & $\lesssim 0.006$ & \\
\hline $200 \times 200$ & d & $r^{1900}$ & 1.31 & 0.40 & -0.10 & 4 & 0 & -3.6 & -3.6 & 1.7 & MBI & 1.0 & 0.99 \\
\hline $200 \times 200$ & d & $r^{1900}$ & 1.31 & 0.40 & -0.10 & 8 & 0 & -3.6 & -3.6 & 1.7 & MBI & 1.4 & 1.4 \\
\hline $200 \times 200$ & d & $r^{1900}$ & 1.31 & 0.40 & -0.10 & 10 & 0 & -3.6 & -3.6 & 1.7 & MBI & 1.4 & 2.1 \\
\hline $200 \times 200$ & d & $r^{1900}$ & 1.31 & 0.40 & -0.10 & 20 & 0 & -3.6 & -3.6 & 1.7 & MBI & 1.5 & 5.6 \\
\hline
\end{tabular}

scale for resistive diffusion across the width of a current sheet, $\tau_{\mathrm{r}}=a^{2} /\left(c_{\mathrm{A}} \delta x\right)$, modified by the ratio of the sound speed and the Alfvén velocity. This interpretation has the nice property that it is consistent with the fact that the magnetic Reynolds number is proportional to the grid resolution.

\section{Appendix B: List of models}

In this appendix we provide a list of the models:

- Table B.1: this table contains a list of 2D models having a positive entropy gradient. Their initial rotation profile is given by
$\Omega_{0}=1900 \mathrm{~s}^{-1}$ and $\alpha_{\Omega}=-1.25$, and their initial magnetic field is uniform.

- Table B.2: this table contains a list of 2D models having a negative entropy gradient. The models rotate initially rigidly or differentially. The initial magnetic field is uniform.

- Table B.3 and Table B.4: this table contains a list of 3D models having different initial magnetic field strengths, entropy gradients, initial rotation laws, and simulated in computational boxes of various size with both types (with and without velocity damping) of radial boundary conditions. 
Table B.3. List of 3D models. The first column (from left to right) gives the geometry of the initial field ("U": uniform; "V" zero-flux). The next two columns show the box size $\left(L_{\varpi} \times L_{\phi} \times L_{z}\right)$ and the number of grid zones $\left(m_{\varpi} \times m_{\phi} \times m_{z}\right)$. The next four columns list the rotation law ("d": differential rotation with $\Omega_{0}=1900 \mathrm{~s}^{-1}$ and $\alpha_{\Omega}=-1.25 ;$; $r^{2}$ " rigid rotation with an angular velocity of $\left.\Omega\right)$, the entropy gradient, $\partial_{\varpi}\left(s_{0}=0.2\right)$, the strength of the initial magnetic field, $b_{0}^{z}$, and the type of boundary condition which was applied (d: velocity damping; p: periodic). The remaining four columns give the growth rate of the MRI, $\sigma$, and the Maxwell stress component $M_{\varpi \phi}^{\text {term }}$ at MRI termination, its maximum value, and its time averaged value, respectively.

\begin{tabular}{|c|c|c|c|c|c|c|c|c|c|c|}
\hline Field & $\begin{array}{c}\text { Grid size } \\
{\left[\mathrm{km}^{3}\right]}\end{array}$ & Resolution & Rot & $\begin{array}{c}\partial_{\varpi} S \\
{\left[\mathrm{~km}^{-1}\right]}\end{array}$ & $\begin{array}{c}b_{0}^{z} \\
{\left[10^{12} \mathrm{G}\right]}\end{array}$ & $\mathrm{BC}$ & $\left.\begin{array}{c}\sigma \\
{\left[\mathrm{ms}^{-1}\right.}\end{array}\right]$ & $\begin{array}{c}M_{\varpi \phi}^{\text {term }} \\
{\left[10^{28} \mathrm{G}^{2} \mathrm{~cm}^{-3}\right]}\end{array}$ & $\begin{array}{c}M_{\varpi \phi}^{\max } \\
{\left[10^{28} \mathrm{G}^{2} \mathrm{~cm}^{-3}\right]}\end{array}$ & $\begin{array}{c}\left\langle M_{\varpi \phi}\right\rangle \\
{\left[10^{28} \mathrm{G}^{2} \mathrm{~cm}^{-3}\right]}\end{array}$ \\
\hline \multirow[t]{50}{*}{$\mathrm{U3}$} & $0.5 \times 0.25 \times 0.5$ & $26 \times 12 \times 26$ & $\mathrm{~d}$ & 0 & 40 & $\mathrm{~d}$ & 0.98 & 0.47 & 298 & 47 \\
\hline & $0.5 \times 0.5 \times 0.5$ & $26 \times 26 \times 26$ & d & 0 & 40 & d & 0.96 & 0.45 & 211 & 36 \\
\hline & $0.5 \times 1 \times 0.5$ & $26 \times 50 \times 26$ & d & 0 & 40 & $\mathrm{~d}$ & 0.96 & 0.45 & 16 & 2.9 \\
\hline & $0.5 \times 2 \times 0.5$ & $26 \times 100 \times 26$ & $\mathrm{~d}$ & 0 & 40 & $\mathrm{~d}$ & 0.92 & 0.44 & 5.0 & 1.5 \\
\hline & $1 \times 0.5 \times 1$ & $50 \times 26 \times 50$ & $\mathrm{~d}$ & 0 & 20 & $\mathrm{~d}$ & 1.05 & 3.1 & 924 & 69 \\
\hline & $1 \times 0.5 \times 1$ & $50 \times 26 \times 50$ & $\mathrm{~d}$ & 0 & 40 & $\mathrm{~d}$ & 1.10 & 3.7 & 553 & 68 \\
\hline & $1 \times 1 \times 1$ & $50 \times 50 \times 50$ & $\mathrm{~d}$ & 0 & 10 & d & 0.76 & 1.5 & 2.7 & 1.1 \\
\hline & $1 \times 1 \times 1$ & $50 \times 50 \times 50$ & $\mathrm{~d}$ & 0 & 20 & $\mathrm{~d}$ & 1.03 & 3.1 & 347 & 44 \\
\hline & $1 \times 1 \times 1$ & $50 \times 50 \times 50$ & d & 0 & 40 & d & 1.09 & 3.1 & 482 & 63 \\
\hline & $1 \times 2 \times 1$ & $50 \times 50 \times 50$ & d & 0 & 10 & d & 0.75 & 1.3 & 1.3 & 0.47 \\
\hline & $1 \times 2 \times 1$ & $50 \times 50 \times 50$ & $\mathrm{~d}$ & 0 & 20 & d & 1.03 & 3.0 & 6.5 & 2.9 \\
\hline & $1 \times 2 \times 1$ & $50 \times 50 \times 50$ & $\mathrm{~d}$ & 0 & 40 & d & 1.09 & 2.9 & 22 & 5.8 \\
\hline & $1 \times 2 \times 1$ & $50 \times 100 \times 50$ & $\mathrm{~d}$ & 0 & 20 & d & 1.02 & 3.1 & 9.5 & 3.4 \\
\hline & $1 \times 2 \times 1$ & $50 \times 100 \times 50$ & $\mathrm{~d}$ & 0 & 40 & d & 1.09 & 2.8 & 60 & 9.2 \\
\hline & $1 \times 4 \times 1$ & $50 \times 200 \times 50$ & $\mathrm{~d}$ & 0 & 20 & d & 1.03 & 2.9 & 4.4 & 2.3 \\
\hline & $1 \times 4 \times 1$ & $50 \times 200 \times 50$ & $\mathrm{~d}$ & 0 & 40 & d & 1.08 & 3.0 & 10.5 & 3.4 \\
\hline & $1 \times 4 \times 1$ & $50 \times 200 \times 50$ & d & 0 & 80 & $\mathrm{~d}$ & 1.01 & 2.7 & 45 & 9.0 \\
\hline & $1 \times 4 \times 1$ & $100 \times 400 \times 100$ & $\mathrm{~d}$ & 0 & 20 & $\mathrm{~d}$ & 1.05 & 3.5 & 6.5 & 2.5 \\
\hline & $1 \times 4 \times 1$ & $100 \times 400 \times 100$ & $\mathrm{~d}$ & 0 & 40 & $\mathrm{~d}$ & 1.09 & 3.5 & 28 & 8.2 \\
\hline & $0.5 \times 0.25 \times 1$ & $26 \times 12 \times 50$ & $\mathrm{~d}$ & 0 & 40 & $\mathrm{~d}$ & 1.04 & 0.44 & 325 & 64 \\
\hline & $0.5 \times 0.5 \times 1$ & $26 \times 26 \times 50$ & $\mathrm{~d}$ & 0 & 40 & d & 1.03 & 0.52 & 227 & 40 \\
\hline & $0.5 \times 1 \times 1$ & $26 \times 50 \times 50$ & $\mathrm{~d}$ & 0 & 40 & $\mathrm{~d}$ & 1.00 & 0.42 & 298 & 33 \\
\hline & $0.5 \times 2 \times 1$ & $26 \times 100 \times 50$ & $\mathrm{~d}$ & 0 & 40 & $\mathrm{~d}$ & 0.96 & 0.50 & 289 & 38 \\
\hline & $1 \times 0.25 \times 0.5$ & $50 \times 12 \times 26$ & d & 0 & 40 & $\mathrm{~d}$ & 1.08 & 2.9 & 310 & 59 \\
\hline & $1 \times 0.38 \times 0.5$ & $50 \times 18 \times 26$ & $\mathrm{~d}$ & 0 & 40 & $\mathrm{~d}$ & 1.08 & 3.0 & 234 & 45 \\
\hline & $1 \times 0.5 \times 0.5$ & $50 \times 26 \times 26$ & $\mathrm{~d}$ & 0 & 40 & $\mathrm{~d}$ & 1.07 & 2.9 & 16 & 6.2 \\
\hline & $1 \times 1 \times 0.5$ & $50 \times 50 \times 26$ & $\mathrm{~d}$ & 0 & 40 & $\mathrm{~d}$ & 1.07 & 3.1 & 9.0 & 2.3 \\
\hline & $1 \times 2 \times 0.5$ & $50 \times 100 \times 26$ & $\mathrm{~d}$ & 0 & 40 & $\mathrm{~d}$ & 1.06 & 3.0 & 3.4 & 1.8 \\
\hline & $0.5 \times 0.25 \times 0.5$ & $26 \times 12 \times 26$ & $\mathrm{~d}$ & 0 & 20 & $\mathrm{p}$ & 1.07 & 97 & 2940 & 145 \\
\hline & $0.5 \times 0.5 \times 0.5$ & $26 \times 26 \times 26$ & $\mathrm{~d}$ & 0 & 20 & $\mathrm{p}$ & 0.99 & 64 & 4196 & 335 \\
\hline & $0.5 \times 1 \times 0.5$ & $26 \times 50 \times 26$ & d & 0 & 20 & $\mathrm{p}$ & 1.06 & 60 & 535 & 19 \\
\hline & $0.5 \times 2 \times 0.5$ & $26 \times 100 \times 26$ & $\mathrm{~d}$ & 0 & 20 & $\mathrm{p}$ & 1.08 & 66 & 66 & 8.7 \\
\hline & $1 \times 0.25 \times 1$ & $50 \times 12 \times 50$ & $\mathrm{~d}$ & 0 & 20 & $\mathrm{p}$ & 1.02 & 13 & 1316 & 110 \\
\hline & $1 \times 0.5 \times 1$ & $50 \times 26 \times 50$ & $\mathrm{~d}$ & 0 & 20 & $\mathrm{p}$ & 1.02 & 8.8 & 1424 & 60 \\
\hline & $1 \times 1 \times 1$ & $50 \times 50 \times 50$ & $\mathrm{~d}$ & 0 & 20 & $\mathrm{p}$ & 0.98 & 42 & 2570 & 128 \\
\hline & $1 \times 2 \times 1$ & $50 \times 100 \times 50$ & $\mathrm{~d}$ & 0 & 20 & $\mathrm{p}$ & 1.04 & 16.5 & 736 & 79 \\
\hline & $1 \times 4 \times 1$ & $50 \times 200 \times 50$ & d & 0 & 20 & $\mathrm{p}$ & 1.05 & 22.5 & 59 & 6.5 \\
\hline & $1 \times 4 \times 1$ & $50 \times 200 \times 50$ & d & 0 & 40 & $\mathrm{p}$ & 1.09 & 40.4 & 361 & 18 \\
\hline & $1 \times 4 \times 1$ & $50 \times 200 \times 50$ & $\mathrm{~d}$ & 0 & 80 & $\mathrm{p}$ & 1.09 & 254 & 254 & 5.5 \\
\hline & $1 \times 4 \times 1$ & $100 \times 400 \times 100$ & $\mathrm{~d}$ & 0 & 20 & $\mathrm{p}$ & 1.08 & 14.9 & 27.6 & 6.4 \\
\hline & $1 \times 4 \times 1$ & $100 \times 400 \times 100$ & $\mathrm{~d}$ & 0 & 40 & $\mathrm{p}$ & 1.11 & 88 & 88 & 11 \\
\hline & $0.5 \times 0.25 \times 1$ & $26 \times 12 \times 50$ & $\mathrm{~d}$ & 0 & 20 & $\mathrm{p}$ & 1.04 & 64 & 2182 & 146 \\
\hline & $0.5 \times 0.25 \times 1$ & $26 \times 26 \times 50$ & $\mathrm{~d}$ & 0 & 20 & $\mathrm{p}$ & 1.03 & 31 & 1540 & 170 \\
\hline & $0.5 \times 1 \times 1$ & $26 \times 50 \times 50$ & $\mathrm{~d}$ & 0 & 20 & $\mathrm{p}$ & 1.01 & 8.9 & 1735 & 102 \\
\hline & $0.5 \times 2 \times 1$ & $26 \times 100 \times 50$ & $\mathrm{~d}$ & 0 & 20 & $\mathrm{p}$ & 1.05 & 30 & 825 & 72 \\
\hline & $0.5 \times 4 \times 1$ & $26 \times 200 \times 50$ & $\mathrm{~d}$ & 0 & 20 & $\mathrm{p}$ & 1.04 & 27 & 103 & 15 \\
\hline & $1 \times 0.25 \times 0.5$ & $50 \times 12 \times 26$ & $\mathrm{~d}$ & 0 & 20 & $\mathrm{p}$ & 1.07 & 32 & 1654 & 171 \\
\hline & $1 \times 0.5 \times 0.5$ & $50 \times 26 \times 26$ & $\mathrm{~d}$ & 0 & 20 & $\mathrm{p}$ & 1.06 & 8.5 & 2902 & 170 \\
\hline & $1 \times 1 \times 0.5$ & $50 \times 50 \times 26$ & $\mathrm{~d}$ & 0 & 20 & $\mathrm{p}$ & 1.05 & 14 & 682 & 13 \\
\hline & $1 \times 2 \times 0.5$ & $50 \times 100 \times 26$ & $\mathrm{~d}$ & 0 & 20 & $\mathrm{p}$ & 1.06 & 14 & 14 & 4.0 \\
\hline
\end{tabular}


Table B.4. Continuation of Table B.3. For the MBI model with $b_{0}^{z}=10 \mathrm{G}$, we do not provide values for the Maxwell stress, because the magnetic field behaves similar to a purely passive field.

\begin{tabular}{|c|c|c|c|c|c|c|c|c|c|c|}
\hline Field & $\begin{array}{c}\text { Grid size } \\
{\left[\mathrm{km}^{3}\right]}\end{array}$ & Resolution & Rot & $\begin{array}{c}\partial_{\varpi} S \\
{\left[\mathrm{~km}^{-1}\right]}\end{array}$ & $\begin{array}{c}b_{0}^{z} \\
{\left[10^{12} \mathrm{G}\right.}\end{array}$ & $\overline{\mathrm{BC}}$ & $\begin{array}{c}\sigma \\
{\left[\mathrm{ms}^{-1}\right.}\end{array}$ & $\begin{array}{c}M_{\varpi \phi}^{\text {term }} \\
{\left[10^{28} \mathrm{G}^{2} \mathrm{~cm}^{-3}\right]}\end{array}$ & $\begin{array}{c}M_{\varpi \phi}^{\max } \\
{\left[10^{28} \mathrm{G}^{2} \mathrm{~cm}^{-3}\right]}\end{array}$ & $\begin{array}{c}\left\langle M_{\varpi \phi}\right\rangle \\
{\left[10^{28} \mathrm{G}^{2} \mathrm{~cm}^{-3}\right.}\end{array}$ \\
\hline \multirow[t]{7}{*}{$\overline{\mathrm{V} 3}$} & $1 \times 1 \times 1$ & $50 \times 50 \times 50$ & $\bar{d}$ & 0 & 10 & $\bar{d}$ & 0.66 & 0.13 & 0.24 & 0.16 \\
\hline & $1 \times 1 \times 1$ & $50 \times 50 \times 50$ & $\mathrm{~d}$ & 0 & 20 & d & 0.96 & 0.53 & 0.53 & 0.21 \\
\hline & $1 \times 1 \times 1$ & $50 \times 50 \times 50$ & $\mathrm{~d}$ & 0 & 40 & d & 1.03 & 1.5 & 1.5 & 0.27 \\
\hline & $1 \times 4 \times 1$ & $50 \times 200 \times 50$ & $\mathrm{~d}$ & 0 & 10 & $\mathrm{~d}$ & 0.66 & 0.13 & 0.52 & 0.24 \\
\hline & $1 \times 4 \times 1$ & $50 \times 200 \times 50$ & d & 0 & 20 & $\mathrm{~d}$ & 0.90 & 0.36 & 0.80 & 0.43 \\
\hline & $1 \times 4 \times 1$ & $100 \times 400 \times 100$ & $\mathrm{~d}$ & 0 & 20 & $\mathrm{~d}$ & 1.08 & 1.1 & 1.1 & 0.20 \\
\hline & $1 \times 4 \times 1$ & $100 \times 400 \times 100$ & $\mathrm{~d}$ & 0 & 20 & $\mathrm{p}$ & 1.08 & 1.5 & 1.5 & 0.21 \\
\hline \multirow[t]{7}{*}{ U3 } & $1 \times 2 \times 1$ & $26 \times 50 \times 26$ & $\mathrm{~d}$ & -0.038 & 20 & d & 1.5 & 1.9 & 5.3 & 0.83 \\
\hline & $1 \times 2 \times 1$ & $26 \times 50 \times 26$ & $\mathrm{~d}$ & -0.038 & 40 & d & 1.7 & 4.1 & 7.2 & 1.1 \\
\hline & $1 \times 2 \times 1$ & $50 \times 100 \times 50$ & $\mathrm{~d}$ & -0.038 & 20 & d & 1.7 & 3.1 & 3.1 & 0.16 \\
\hline & $1 \times 2 \times 1$ & $50 \times 100 \times 50$ & $\mathrm{~d}$ & -0.038 & 40 & $\mathrm{~d}$ & 1.9 & 4.4 & 4.4 & 0.23 \\
\hline & $1 \times 2 \times 1$ & $50 \times 100 \times 50$ & $r^{1900}$ & -0.10 & $10^{-11}$ & d & 2.6 & & & \\
\hline & $1 \times 2 \times 1$ & $50 \times 100 \times 50$ & $r^{1900}$ & -0.10 & 0.01 & $\mathrm{~d}$ & 2.6 & $2.3 \times 10^{-8}$ & 0.11 & 0.040 \\
\hline & $1 \times 2 \times 1$ & $50 \times 100 \times 50$ & $r^{1900}$ & -0.10 & 20 & d & 2.6 & 0.060 & 0.87 & 0.30 \\
\hline V3 & $1 \times 2 \times 1$ & $50 \times 100 \times 50$ & $\mathrm{~d}$ & -0.038 & 20 & $\mathrm{~d}$ & 1.6 & 0.54 & 0.54 & 0.011 \\
\hline
\end{tabular}

\section{References}

Acheson, D. J. 1978, Roy. Soc. London Phil. Trans. Ser. A, 289, 459

Akiyama, S., Wheeler, J. C., Meier, D. L., \& Lichtenstadt, I. 2003, ApJ, 584, 954

Aloy, M. A., \& Obergaulinger, M. 2007, in Rev. Mex. Astron. Astrofis. Conf. Ser., 30, 96

Balbus, S. A. 1995, ApJ, 453, 380

Balbus, S. A., \& Hawley, J. F. 1991, ApJ, 376, 214

Balbus, S. A., \& Hawley, J. F. 1992, ApJ, 392, 662

Balbus, S. A., \& Hawley, J. F. 1998, Rev. Mod. Phys., 70, 1

Biskamp, D. 2000, Magnetic Reconnection in Plasmas Magnetic reconnection in plasmas, Cambridge monographs on plasma physics (Cambridge, UK: Cambridge University Press), 3, 387

Bodo, G., Mignone, A., Cattaneo, F., Rossi, P., \& Ferrari, A. 2008, ArXiv e-prints, 805

Burrows, A., Dessart, L., Livne, E., Ott, C. D., \& Murphy, J. 2007, ApJ, 664, 416

Chandrasekhar, S. 1960, PNAS, 46, 253

Evans, C. R., \& Hawley, J. F. 1988, ApJ, 332, 659

Fromang, S., \& Papaloizou, J. 2007, A\&A, 476, 1113

Goodman, J., \& Xu, G. 1994, ApJ, 432, 213

Harten, A. 1983, J. Comput. Phys., 49, 357

Hawley, J. F., \& Balbus, S. A. 1992, ApJ, 400, 595

Heger, A., Woosley, S. E., \& Spruit, H. C. 2005, ApJ, 626, 350
Janka, H.-T., Langanke, K., Marek, A., Martínez-Pinedo, G., \& Müller, B. 2007, Phys. Rep., 442, 38

Keil, W., Janka, H.-T., \& Müller, E. 1996, ApJ, 473, L111

Klahr, H. H., \& Bodenheimer, P. 2003, ApJ, 582, 869

Kotake, K., Yamada, S., Sato, K., et al. 2004, Phys. Rev. D, 69, 124004

Lesur, G., \& Ogilvie, G. I. 2008, A\&A, 488, 451

LeVeque, R. J. 1992, Numerical Methods for Conservation Laws, 2nd edn., Lectures in Mathematics, ETH Zürich (Birkhäuser)

Levy, D., Puppo, G., \& Russo, G. 2002, SIAM J. Sci. Comput., 24, 480

Obergaulinger, M., Aloy, M. A., Dimmelmeier, H., \& Müller, E. 2006a, A\&A, 457, 209

Obergaulinger, M., Aloy, M. A., \& Müller, E. 2006b, A\&A, 450, 1107

Parker, E. N. 1966, ApJ, 145, 811

Pessah, M. E., Chan, C.-k., \& Psaltis, D. 2007, ApJ, 668, L51

Regev, O., \& Umurhan, O. M. 2008, A\&A, 481, 21

Sano, T., \& Inutsuka, S.-I. 2001, ApJ, 561, L179

Shakura, N. I., \& Syunyaev, R. A. 1973, A\&A, 24, 337

Suresh, A., \& Huynh, H. 1997, J. Comput. Phys., 136, 83

Takiwaki, T., Kotake, K., Nagataki, S., \& Sato, K. 2004, ApJ, 616, 1086

Tassoul, J. 1978, Theory of rotating stars, Princeton Series in Astrophysics (Princeton: University Press)

Thompson, T. A., Quataert, E., \& Burrows, A. 2005, ApJ, 620, 861

Toro, E. F., \& Titarev, V. A. 2006, J. Comput. Phys., 216, 403

Urpin, V. A. 1996, MNRAS, 280, 149

Velikhov, E. 1959, Sov. Phys. JETP, 36, 995

Yamada, S., \& Sawai, H. 2004, ApJ, 608, 907 